ESAIM: M2AN 48 (2014) 347-386

DOI: $10.1051 / \mathrm{m} 2 \mathrm{an} / 2013111$
ESAIM: Mathematical Modelling and Numerical Analysis

www.esaim-m2an.org

\title{
ON A VARIANT OF RANDOM HOMOGENIZATION THEORY: CONVERGENCE OF THE RESIDUAL PROCESS AND APPROXIMATION OF THE HOMOGENIZED COEFFICIENTS
}

\author{
FrédÉRIC LEGOLL ${ }^{1}$ AND Florian ThOMINES ${ }^{2}$
}

\begin{abstract}
We consider the variant of stochastic homogenization theory introduced in $[\mathrm{X}$. Blanc, C. Le Bris and P.-L. Lions, C. R. Acad. Sci. Série I 343 (2006) 717-724.; X. Blanc, C. Le Bris and P.-L. Lions, J. Math. Pures Appl. 88 (2007) 34-63.]. The equation under consideration is a standard linear elliptic equation in divergence form, where the highly oscillatory coefficient is the composition of a periodic matrix with a stochastic diffeomorphism. The homogenized limit of this problem has been identified in [X. Blanc, C. Le Bris and P.-L. Lions, C. R. Acad. Sci. Série I 343 (2006) 717-724.]. We first establish, in the one-dimensional case, a convergence result (with an explicit rate) on the residual process, defined as the difference between the solution to the highly oscillatory problem and the solution to the homogenized problem. We next return to the multidimensional situation. As often in random homogenization, the homogenized matrix is defined from a so-called corrector function, which is the solution to a problem set on the entire space. We describe and prove the almost sure convergence of an approximation strategy based on truncated versions of the corrector problem.
\end{abstract}

Mathematics Subject Classification. 35R60, 35B27, 60H, 60F05.

Revised June 19, 2013. Accepted September 9, 2013

Published online February 7, 2014.

\section{INTRODUCTION}

Homogenization theory for linear second-order elliptic equations with highly oscillatory coefficients is a well developed topic. In the periodic case, the homogenized problem is known, and convergence rates of the oscillatory solution (denoted $u^{\varepsilon}$ ) towards the homogenized solution $u_{\star}$ have been obtained.

The situation is less clear in the random (say stationary ergodic) setting. The convergence of $u^{\varepsilon}(\cdot, \omega)$ to some deterministic $u_{\star}$ is a classical result. However, rates of convergence are much more difficult to obtain. A central difficulty in stochastic homogenization is that the corrector problem, which needs to be solved to next compute the homogenized matrix, is set on the entire space (in contrast with the periodic case, where it is set on the periodic cell). This induces many theoretical and practical difficulties.

\footnotetext{
Keywords and phrases. Stochastic homogenization, random stationary diffeomorphisms, central limit result, approximation of homogenized coefficients.

1 Laboratoire Navier, École Nationale des Ponts et Chaussées, Université Paris-Est, 6 et 8 Avenue Blaise Pascal, 77455 MarneLa-Vallée Cedex 2, France. legoll@lami.enpc.fr

2 INRIA Rocquencourt, MICMAC team-project, Domaine de Voluceau, B.P. 105, 78153 Le Chesnay Cedex, France. florian. thomines@enpc.fr
} 
In what follows, we are interested in the problem

$$
-\operatorname{div}\left[A\left(\frac{x}{\varepsilon}, \omega\right) \nabla u^{\varepsilon}(x, \omega)\right]=f(x) \text { in } \mathcal{D}, \quad u^{\varepsilon}(\cdot, \omega)=0 \text { on } \partial \mathcal{D},
$$

where the random matrix $A$ satisfies standard coercivity and boundedness properties (and some structure assumptions that we detail below), $\mathcal{D}$ is an open bounded set of $\mathbb{R}^{d}$ and $f \in L^{2}(\mathcal{D})$.

The analysis of the residual, which we define as the difference between the oscillatory solution $u^{\varepsilon}$ and the homogenized solution $u_{\star}$, was first taken up in [9], and next complemented in [3]. Both studies consider the equation $-\frac{\mathrm{d}}{\mathrm{d} x}\left[a\left(\frac{x}{\varepsilon}, \omega\right) \frac{\mathrm{d} u^{\varepsilon}}{\mathrm{d} x}\right]=f(x)$ in the one-dimensional setting, where $a(x, \omega)$ is a random stationary process. The behavior, when $\varepsilon \rightarrow 0$, of the residual $u^{\varepsilon}(x, \omega)-u_{\star}(x)$ turns out to depend on the asymptotic behavior of the correlation function $\eta(x):=\operatorname{Cov}(a(0, \cdot), a(x, \cdot))$ of the conductivity coefficient. In [9], the case of small correlation lengths is studied, which amounts to assuming that $\eta(x) \sim_{x \rightarrow \infty} x^{-\alpha}$ with $\alpha>1$. The correlation function is thus integrable. In that case, when $\varepsilon \rightarrow 0$, the random process $\frac{u^{\varepsilon}(x, \omega)-u_{\star}(x)}{\sqrt{\varepsilon}}$ converges in distribution to a Gaussian random process. The case of long correlation lengths, namely when $\eta(x) \sim_{x \rightarrow \infty} x^{-\alpha}$ for some $0<\alpha<1$, is studied in [3], where it is shown that the random process $\frac{u^{\varepsilon}(x, \omega)-u_{\star}(x)}{\varepsilon^{\alpha / 2}}$ converges in distribution to a Gaussian random process (defined using a fractional Brownian motion). This result shows that the rate of convergence of $u^{\varepsilon}$ to $u_{\star}$ can be as slow as $\varepsilon^{\alpha / 2}$ for any $\alpha>0$, if no further assumptions on the stationary process $a$ are made.

Of course, in both works, the one-dimensional setting allows to get some analytical expression for the residual. In turn, the analysis of the asymptotic behavior of the residual performed in $[3,9]$ relies on this analytical expression. In higher dimensions, the case of the equation $-\Delta u^{\varepsilon}+q\left(\frac{x}{\varepsilon}, \omega\right) u^{\varepsilon}=f(x)$ has been studied in [2].

Our first aim here is to study a similar question for a variant of the classical stochastic homogenization theory. We consider in the sequel the following problem, which has been introduced in [7] and further studied in [8]:

$$
-\operatorname{div}\left[A_{\text {per }}\left(\phi^{-1}\left(\frac{x}{\varepsilon}, \omega\right)\right) \nabla u^{\varepsilon}(x, \omega)\right]=f(x) \text { in } \mathcal{D}, \quad u^{\varepsilon}(\cdot, \omega)=0 \text { on } \partial \mathcal{D},
$$

where $\phi$ is almost surely a diffeomorphism from $\mathbb{R}^{d}$ to $\mathbb{R}^{d}$ with some stationary properties and $A_{\text {per }}$ is a $\mathbb{Z}^{d}$ periodic matrix, which satisfies the classical coercivity and boundedness properties (see precise assumptions in Sect. 2.1 below). This model is appropriate to represent a periodic, ideal material, that is randomly deformed (think of fibers in a composite material that are placed at a random position, rather than on a perfect, periodic lattice). In [7], it is shown that the solution $u^{\varepsilon}(\cdot, \omega)$ to the above problem converges as $\varepsilon$ goes to 0 to $u_{\star}$, solution to some homogenized problem (see Sect. 2 below). In the sequel, we aim at obtaining the rate of convergence of $u^{\varepsilon}$ to $u_{\star}$, in the one dimensional setting. We make below an assumption on the random diffeomorphism which implies that our setting is close to the one studied in [9] (rather than that studied in [3]). Under this assumption, we show that the random process $\frac{u^{\varepsilon}(x, \omega)-u_{\star}(x)}{\sqrt{\varepsilon}}$ converges in distribution to a Gaussian random process that we completely characterize (see Sect. 3.1, Thm. 3.2).

We next turn to a question of a different nature. As pointed out above, the homogenized matrix $A^{\star}$ associated to (1.2) depends on the solution of the corrector problem (see (2.9) below), which is set on the entire space. Computing an approximation of $A^{\star}$ is thus, in practice, a challenging question. A standard strategy is to consider the corrector problem on a bounded (large) domain $Q_{N}$, supplemented with (say periodic) boundary conditions. An approximation of the exact corrector is thus computed, from which an approximate homogenized matrix $A_{N}^{\star}(\omega)$ is inferred. As a by-product of working on a bounded domain, the approximate homogenized matrix is random. In the classical random homogenization setting (that is (1.1) where $A$ is a stationary matrix), the convergence (and its rate) of $A_{N}^{\star}(\omega)$ to $A^{\star}$ has been studied in [10], using some previous approximation 
results [20]. It is shown there that $A_{N}^{\star}(\omega)$ almost surely converges to $A^{\star}$, and that $\mathbb{E}\left[\left|A_{N}^{\star}-A^{\star}\right|^{2}\right]$ converges to 0 as $N^{-\alpha}$, for some $\alpha>0$ which implicitly depends on the mixing properties of the random coefficient $A$ of the equation (1.1). It is expected that, depending on the properties of that random coefficient, $\alpha$ can be arbitrary small.

In this work, we consider the above variant (1.2) of the classical random homogenization setting. We describe a strategy (originally introduced in [12]) to approximate $A^{\star}$ which is based, as in the classical setting, on solving the corrector problems on bounded domains $Q_{N}$. We prove here the convergence of this approach (see Sect. 3.2, Thm. 3.4).

Our article is articulated as follows. In Section 2, we present in details the variant of the classical random homogenization introduced in $[7,8]$. We next present in Section 3 our two main results, first on the residual process in dimension one (see Sect. 3.1 and Thm. 3.2), second on a practical approximation of the homogenized matrix in dimension $d \geq 2$ (see Sect. 3.2 and Thm. 3.4). The subsequent two sections are devoted to the proof of Theorem 3.2. The actual proof is performed in Section 4, and needs some technical results which are proved in Section 5. Our final section, Section 6, collects the proof of Theorem 3.4.

\section{A VARIANT OF THE CLASSICAL RANDOM HOMOGENIZATION}

To begin with, we introduce the basic setting of stochastic homogenization we employ. We refer to [13] for a general, numerically oriented presentation, and to $[5,11,15]$ for classical textbooks. We also refer to $[7,8]$ for a presentation of our particular setting (see also [1]). Throughout this article, $(\Omega, \mathcal{F}, \mathbb{P})$ is a probability space and we denote by $\mathbb{E}(X)=\int_{\Omega} X(\omega) d \mathbb{P}(\omega)$ the expectation value of any random variable $X \in L^{1}(\Omega, d \mathbb{P})$. For any fixed $d \in \mathbb{N}^{\star}$ (the ambient physical dimension), we assume that the group $\left(\mathbb{Z}^{d},+\right)$ acts on $\Omega$. We denote by $\left(\tau_{k}\right)_{k \in \mathbb{Z}^{d}}$ this action, and assume that it preserves the measure $\mathbb{P}$, that is, for all $k \in \mathbb{Z}^{d}$ and all $B \in \mathcal{F}, \mathbb{P}\left(\tau_{k} B\right)=\mathbb{P}(B)$. We assume that the action $\tau$ is ergodic, that is, if $B \in \mathcal{F}$ is such that $\tau_{k} B=B$ for any $k \in \mathbb{Z}^{d}$, then $\mathbb{P}(B)=0$ or 1 . In addition, we define the following notion of (discrete) stationarity (see $[7,8])$ : any $F \in L_{\text {loc }}^{1}\left(\mathbb{R}^{d}, L^{1}(\Omega)\right)$ is said to be stationary if

$$
\forall k \in \mathbb{Z}^{d}, \quad F(x+k, \omega)=F\left(x, \tau_{k} \omega\right) \quad \text { almost everywhere and almost surely. }
$$

In this setting, the ergodic theorem $[16,18,19]$ can be stated as follows: Let $F \in L^{\infty}\left(\mathbb{R}^{d}, L^{1}(\Omega)\right)$ be a stationary random variable in the above sense. For $k=\left(k_{1}, k_{2}, \ldots, k_{d}\right) \in \mathbb{Z}^{d}$, we set $|k|_{\infty}=\sup _{1 \leq i \leq d}\left|k_{i}\right|$. Then

$$
\frac{1}{(2 N+1)^{d}} \sum_{|k|_{\infty} \leq N} F\left(x, \tau_{k} \omega\right) \underset{N \rightarrow \infty}{\longrightarrow} \mathbb{E}(F(x, \cdot)) \quad \text { in } L^{\infty}\left(\mathbb{R}^{d}\right) \text {, almost surely. }
$$

This implies (denoting by $Q=(0,1)^{d}$ the unit cube in $\mathbb{R}^{d}$ ) that

$$
F\left(\frac{x}{\varepsilon}, \omega\right) \underset{\varepsilon \rightarrow 0}{\stackrel{*}{\longrightarrow}} \mathbb{E}\left(\int_{Q} F(x, \cdot) \mathrm{d} x\right) \quad \text { in } L^{\infty}\left(\mathbb{R}^{d}\right), \text { almost surely. }
$$

\subsection{Mathematical setting and homogenization result}

As pointed out in the introduction, we consider in this article the following problem, which has been introduced in [7] and further studied in [8]:

$$
-\operatorname{div}\left[A_{\text {per }}\left(\phi^{-1}\left(\frac{x}{\varepsilon}, \omega\right)\right) \nabla u^{\varepsilon}(x, \omega)\right]=f(x) \text { in } \mathcal{D}, \quad u^{\varepsilon}(\cdot, \omega)=0 \text { on } \partial \mathcal{D},
$$


where $\mathcal{D}$ is a bounded open set of $\mathbb{R}^{d}, f \in L^{2}(\mathcal{D}), \phi$ is almost surely a diffeomorphism from $\mathbb{R}^{d}$ to $\mathbb{R}^{d}$, and $A_{\text {per }}$ is a $\mathbb{Z}^{d}$-periodic matrix, that satisfies the classical coercivity and boundedness properties: there exists $a^{+} \geq a_{-}>0$ such that

$$
\forall \xi \in \mathbb{R}^{d}, \quad a_{-}|\xi|^{2} \leq A_{\text {per }}(x) \xi \cdot \xi \text { almost everywhere on } \mathbb{R}^{d} \text {, and } a^{+}=\left\|A_{\text {per }}\right\|_{L^{\infty}\left(\mathbb{R}^{d}\right)}<\infty .
$$

In addition, we assume that the map $\phi(\cdot, \omega)$ satisfies

$$
\begin{gathered}
\underset{\omega \in \Omega, x \in \mathbb{R}^{d}}{\operatorname{EssInf}}(\operatorname{det}(\nabla \phi(x, \omega)))=\nu>0, \\
\operatorname{EssSup}_{\omega \in \Omega, x \in \mathbb{R}^{d}}|\nabla \phi(x, \omega)|=M<+\infty,
\end{gathered}
$$

$\nabla \phi$ is stationary in the sense of $(2.1)$.

Assumptions (2.4) and (2.5) mean that $\phi$ is a well-behaved diffeomorphism, uniformly in $\omega$. Note that $A_{\text {per }} \circ \phi^{-1}$ is in general not stationary. The above setting is thus not a particular case of the classical stationary setting.

In [7], it is shown that, under the above conditions, $u^{\varepsilon}(\cdot, \omega)$ converges to $u_{\star}$ almost surely (strongly in $L^{2}(\mathcal{D}$ ) and weakly in $\left.H^{1}(\mathcal{D})\right)$ when $\varepsilon$ goes to 0 , where $u_{\star}$ is the solution to the homogenized problem

$$
-\operatorname{div}\left[A^{\star} \nabla u_{\star}(x)\right]=f(x) \text { in } \mathcal{D}, \quad u_{\star}=0 \text { on } \partial \mathcal{D} .
$$

In $(2.7)$, the homogenized matrix coefficient $A^{\star}$ is equal to

$$
\forall 1 \leq i, j \leq d, \quad A_{i j}^{\star}=\operatorname{det}\left(\mathbb{E}\left(\int_{Q} \nabla \phi(y, \cdot) \mathrm{d} y\right)\right)^{-1} \mathbb{E}\left(\int_{\phi(Q, \cdot)} e_{i}^{T} A_{\mathrm{per}}\left(\phi^{-1}(y, \cdot)\right)\left(e_{j}+\nabla w_{e_{j}}(y, \cdot)\right) \mathrm{d} y\right),
$$

where $Q=(0,1)^{d}$ and where, for all $p \in \mathbb{R}^{d}, w_{p}$ solves the following corrector problem:

$$
\left\{\begin{array}{l}
-\operatorname{div}\left[A_{\text {per }}\left(\phi^{-1}(y, \omega)\right)\left(p+\nabla w_{p}(y, \omega)\right)\right]=0 \text { in } \mathbb{R}^{d}, \\
w_{p}(y, \omega)=\widetilde{w}_{p}\left(\phi^{-1}(y, \omega), \omega\right), \quad \nabla \widetilde{w}_{p} \text { is stationary in the sense of }(2.1), \\
\mathbb{E}\left(\int_{\phi(Q, \cdot)} \nabla w_{p}(y, \cdot) \mathrm{d} y\right)=0 .
\end{array}\right.
$$

\subsection{The one-dimensional case}

Our first main result, presented in Section 3.1, is a convergence result in the one-dimensional case. In that setting, it is possible to write some explicit formulas. Choosing $\mathcal{D}=(0,1)$, the problems $(2.2)$ and (2.7) respectively read

$$
-\frac{\mathrm{d}}{\mathrm{d} x}\left[a_{\text {per }}\left(\phi^{-1}\left(\frac{x}{\varepsilon}, \omega\right)\right) \frac{\mathrm{d} u^{\varepsilon}}{\mathrm{d} x}(x, \omega)\right]=f(x) \text { in }(0,1), \quad u^{\varepsilon}(0, \omega)=0, \quad u^{\varepsilon}(1, \omega)=0,
$$

and

$$
-\frac{\mathrm{d}}{\mathrm{d} x}\left(a^{\star} \frac{d u_{\star}}{\mathrm{d} x}(x)\right)=f(x) \text { in }(0,1), \quad u_{\star}(0)=0, \quad u_{\star}(1)=0 .
$$

The corrector problem (2.9), which reads

$$
\left\{\begin{array}{l}
-\frac{\mathrm{d}}{\mathrm{d} y}\left[a_{\text {per }}\left(\phi^{-1}(y, \omega)\right)\left(1+\frac{\mathrm{d} w}{\mathrm{~d} y}(y, \omega)\right)\right]=0 \text { in } \mathbb{R}, \\
w(y, \omega)=\widetilde{w}\left(\phi^{-1}(y, \omega), \omega\right), \quad \frac{\mathrm{d} \widetilde{w}}{\mathrm{~d} y} \text { is stationary in the sense of }(2.1), \\
\mathbb{E}\left(\int_{\phi(Q, \cdot)} \frac{\mathrm{d} w}{\mathrm{~d} y}(y, \cdot) \mathrm{d} y\right)=0,
\end{array}\right.
$$


can be analytically solved. Its solution $w$ satisfies

$$
1+\frac{\mathrm{d} w}{\mathrm{~d} y}(y, \omega)=\frac{a^{\star}}{a_{\mathrm{per}}\left(\phi^{-1}(y, \omega)\right)}
$$

where the homogenized coefficient $a^{\star}$ is given by

$$
\left(a^{\star}\right)^{-1}=\frac{1}{\mathbb{E}\left(\int_{0}^{1} \phi^{\prime}(y, \cdot) \mathrm{d} y\right)} \mathbb{E}\left(\int_{0}^{1} \frac{\phi^{\prime}(y, \cdot)}{a_{\mathrm{per}}(y)} \mathrm{d} y\right) .
$$

As pointed out in [7], we observe on (2.13) that, in the one-dimensional case, the gradient of the corrector has the same structure as the highly oscillatory coefficient in (2.10): it is equal to a periodic function composed with $\phi^{-1}$. This is not the case in dimensions $d \geq 2$, as shown in [7].

\section{Main Results}

In this article, we show the following two main results, Theorems 3.2 and 3.4.

\subsection{Residual process in dimension one}

Our first aim is to characterize how the residual process $u^{\varepsilon}(x, \omega)-u_{\star}(x)$ converges to zero, where $u^{\varepsilon}$ solves (2.10) and $u_{\star}$ solves (2.11). To this aim, we make the following assumptions. Let us introduce the 1-periodic function

$$
\psi(x)=\frac{1}{a_{\text {per }}(x)}-\frac{1}{a^{\star}}
$$

and the random variables

$$
Y_{k}(\omega)=\int_{k}^{k+1} \psi(t) \phi^{\prime}(t, \omega) \mathrm{d} t
$$

As $\psi$ is periodic and $\phi^{\prime}$ is stationary, the random variables $Y_{k}$ are identically distributed. Due to (2.14), we have

$$
\mathbb{E}\left(Y_{0}\right)=\mathbb{E}\left(\int_{0}^{1} \psi(t) \phi^{\prime}(t, \cdot) \mathrm{d} t\right)=0 .
$$

We furthermore assume that the random variables $Y_{k}$ are independent, and hence that

$$
\text { the variables } Y_{k} \text { are i.i.d. }
$$

Likewise, we consider the random variables

$$
D_{k}(\omega)=\int_{k}^{k+1} \phi^{\prime}(t, \omega) \mathrm{d} t
$$

which are identically distributed, and make the assumption that

$$
\text { the variables } D_{k} \text { are i.i.d. }
$$

Remark 3.1. Suppose that the derivative of the random diffeomorphism $\phi$ reads

$$
\phi^{\prime}(y, \omega)=1+\sum_{k \in \mathbb{Z}} X_{k}(\omega) G_{\text {per }}(y) \mathbf{1}_{[k, k+1)}(y),
$$

where $X_{k}(\omega)$ are independent and identically distributed random variables and $G_{\text {per }}$ is a 1-periodic bounded function, such that, for some $0<m<1$,

$$
\left|X_{0}(\omega)\right| \leq m \text { almost surely and }\left\|G_{\text {per }}\right\|_{L^{\infty}(\mathbb{R})} \leq m .
$$

Then, the conditions (2.4), (2.5) and (2.6) are satisfied with $\nu=1-m^{2}>0$ and $M=1+m^{2}$. By construction, the assumptions (3.3) and (3.5) are also fullfilled. 
The first main result of this article is the following theorem, the proof of which is postponed until Section 4.2.

Theorem 3.2. Assume that $a_{\mathrm{per}}$ and $\phi$ satisfy (2.3), (2.4), (2.5) and (2.6). Assume furthermore the independence conditions (3.3) and (3.5). We consider $u^{\varepsilon}$ solution to (2.10) and $u_{\star}$ solution to (2.11). Then the residual process converges in distribution to a Gaussian process,

$$
\frac{u^{\varepsilon}(x, \omega)-u_{\star}(x)}{\sqrt{\varepsilon}} \underset{\varepsilon \rightarrow 0}{\mathcal{L}} G_{0}(x, \omega),
$$

where

$$
G_{0}(x, \omega)=\frac{\sqrt{\mathbb{V a r}\left(Y_{0}\right)}}{\sqrt{\mathbb{E}\left(\int_{0}^{1} \phi^{\prime}\right)}} \int_{0}^{1} K_{0}(x, t) \mathrm{d} W_{t},
$$

where $W_{t}$ denotes the classical Brownian motion and $K_{0}(x, t)$ is given by

$$
K_{0}(x, t)=\left(\mathbf{1}_{[0, x]}(t)-x\right)\left(\int_{0}^{1} F(s) \mathrm{d} s-F(t)\right) \quad \text { with } \quad F(t)=\int_{0}^{t} f(s) \mathrm{d} s .
$$

Remark 3.3. It might be possible to weaken assumptions (3.3) and (3.5), and to only assume that the identically distributed variables $Y_{k}$ are such that $\sum_{k \in \mathbb{Z}}\left|\mathbb{C o v}\left(Y_{0}, Y_{k}\right)\right|<+\infty$, and likewise for $D_{k}$. We however do not pursue in that direction.

\subsection{Approximation of the homogenized matrix}

In this section, we return to the multidimensional setting. To compute the homogenized matrix $A^{\star}$ defined by (2.8), we first need to solve the corrector problem (2.9), which is set on the entire space. In practice, approximations are therefore in order.

In the sequel, we describe a strategy introduced in [12], and that mimicks the approach proposed in [10] to approximate standard corrector problems in classical random homogenization. In this article, we analyze this approach and prove its convergence (see Thm. 3.4 below). This is our second main result. We refer to Section 3.2 of [1] for some illustrative numerical tests.

Convention: Following Lemme 2.1 of [7], we adopt the convention that $[\nabla \phi]_{i j}=\frac{\partial \phi_{i}}{\partial x_{j}}$ for any $1 \leq i, j \leq d$. Hence, for any scalar-valued function $\psi$, the gradient of $\widetilde{\psi}=\psi \circ \phi$ is given by $\nabla \widetilde{\psi}(z)=(\nabla \phi(z))^{T} \nabla \psi(\phi(z))$. This convention implies that $\left[\nabla \phi\left(\phi^{-1}\right)\right] \nabla\left(\phi^{-1}\right)=$ Id.

\subsubsection{Presentation of the approximation}

The weak formulation of the corrector problem (2.9) reads as follows (see [7]): for all $\widetilde{\psi}$ stationary in the sense of (2.1), we have

$$
\mathbb{E}\left(\int_{\phi(Q, \cdot)}(\nabla \psi(y, \omega))^{T} A_{\text {per }}\left(\phi^{-1}(y, \omega)\right)\left(p+\nabla w_{p}(y, \omega)\right) \mathrm{d} y\right)=0,
$$

where $\psi=\widetilde{\psi} \circ \phi^{-1}$. The above expression can be rewritten, after a change of variables, as

$$
\mathbb{E}\left[\int_{Q} \operatorname{det}(\nabla \phi)(\nabla \widetilde{\psi})^{T}(\nabla \phi)^{-1} A_{\text {per }}\left(p+(\nabla \phi)^{-T} \nabla \widetilde{w}_{p}\right)\right]=0
$$


where $(\nabla \phi)^{-T}$ is defined as $\left[(\nabla \phi)^{-1}\right]^{T}$. Since $\widetilde{\psi}, \nabla \phi, A_{\text {per }}$ and $\nabla \widetilde{w}_{p}$ are stationary in the sense of $(2.1)$, the ergodic theorem yields

$$
\lim _{N \rightarrow \infty} \frac{1}{\left|Q_{N}\right|} \int_{Q_{N}} \operatorname{det}(\nabla \phi)(\nabla \widetilde{\psi})^{T}(\nabla \phi)^{-1} A_{\text {per }}\left(p+(\nabla \phi)^{-T} \nabla \widetilde{w}_{p}\right)=0 \quad \text { a.s. }
$$

where $Q_{N}=N Q$. For a fixed $N$, we now define the approximate corrector $\widetilde{w}_{p}^{N}$ as the $Q_{N}$-periodic function satisfying:

$$
\text { for all } \widetilde{\psi} Q_{N} \text {-periodic, } \quad \int_{Q_{N}} \operatorname{det}(\nabla \phi)(\nabla \widetilde{\psi})^{T}(\nabla \phi)^{-1} A_{\text {per }}\left(p+(\nabla \phi)^{-T} \nabla \widetilde{w}_{p}^{N}\right)=0 \text {. }
$$

Note that $\widetilde{w}_{p}^{N}$ is uniquely defined up to an additive constant.

In turn, recall that $A^{\star}$ is defined by (2.8). After a change of variables, we infer from that equation that, for any $1 \leq i, j \leq d$, we have

$$
A_{i j}^{\star}=\operatorname{det}\left(\mathbb{E}\left(\int_{Q} \nabla \phi(y, \cdot) \mathrm{d} y\right)\right)^{-1} \mathbb{E}\left(\int_{Q} \operatorname{det}(\nabla \phi(y, \cdot)) e_{i}^{T} A_{\mathrm{per}}(y)\left(e_{j}+(\nabla \phi)^{-T} \nabla \widetilde{w}_{e_{j}}(y, \cdot)\right) \mathrm{d} y\right) .
$$

The ergodic theorem yields

$$
A_{i j}^{\star}=\lim _{N \rightarrow \infty}\left\{\operatorname{det}\left(\frac{1}{\left|Q_{N}\right|} \int_{Q_{N}} \nabla \phi(\cdot, \omega)\right)^{-1} \frac{1}{\left|Q_{N}\right|} \int_{Q_{N}} \operatorname{det}(\nabla \phi) e_{i}^{T} A_{\text {per }}\left(e_{j}+(\nabla \phi)^{-T} \nabla \widetilde{w}_{e_{j}}\right)\right\} \quad \text { a.s. }
$$

It is thus natural to approximate $A^{\star}$ by the matrix $A_{N}^{\star}(\omega)$ defined by

$$
A_{N}^{\star}(\omega)=\operatorname{det}\left(\frac{1}{\left|Q_{N}\right|} \int_{Q_{N}} \nabla \phi(\cdot, \omega)\right)^{-1} B_{N}^{\star}(\omega)
$$

where the matrix $B_{N}^{\star}(\omega)$ is defined by, for any $1 \leq i, j \leq d$,

$$
\begin{aligned}
{\left[B_{N}^{\star}(\omega)\right]_{i j} } & =\frac{1}{\left|Q_{N}\right|} \int_{Q_{N}} \operatorname{det}(\nabla \phi) e_{i}^{T} A_{\text {per }}\left(e_{j}+(\nabla \phi)^{-T} \nabla \widetilde{w}_{e_{j}}^{N}\right) \\
& =\frac{1}{\left|Q_{N}\right|} \int_{\phi\left(Q_{N}, \omega\right)} e_{i}^{T} A_{\text {per }}\left(\phi^{-1}(y, \omega)\right)\left(e_{j}+\nabla w_{e_{j}}^{N}(y, \omega)\right) \mathrm{d} y,
\end{aligned}
$$

where, for any $p \in \mathbb{R}^{d}, \widetilde{w}_{p}^{N}$ is defined by (3.9) and

$$
w_{p}^{N}(y, \omega)=\widetilde{w}_{p}^{N}\left(\phi^{-1}(y, \omega), \omega\right) .
$$

Note that, as is standard in stochastic homogenization, the approximation $A_{N}^{\star}(\omega)$ is a random matrix, even though the exact homogenized matrix $A^{\star}$ is deterministic. This is a by-product of working on the truncated domain $Q_{N}$ rather than $\mathbb{R}^{d}$.

\subsubsection{Convergence of the approach}

We prove in Section 6 below the following convergence result:

Theorem 3.4. Let $\phi$ be a diffeomorphism satisfying (2.4), (2.5) and (2.6), and $A_{\mathrm{per}}$ be a periodic matrix that satisfies the ellipticity condition (2.3). Then the random matrix $A_{N}^{\star}(\omega)$ defined by (3.10) converges almost surely to the deterministic homogenized matrix $A^{\star}$ defined by (2.8) when $N \rightarrow \infty$ :

$$
\lim _{N \rightarrow \infty} A_{N}^{\star}(\omega)=A^{\star} \quad \text { almost surely. }
$$




\section{Asymptotic Behavior of the Residual}

The aim of this Section and of the next one is to prove our first main result, Theorem 3.2 of Section 3.1. Using the one dimensional setting, we first establish a "representation" formula for the residual (see Sect. 4.1, Thm. 4.2). Using this formula, we are next in position to study the asymptotic behavior of the residual when $\varepsilon \rightarrow 0$ (see Sect. 4.2). Section 5 collects the proofs of some technical results used in Sections 4.1 and 4.2.

\subsection{Representation formulas}

The following technical result will be useful in the sequel. Its proof is postponed until Section 5.1.

Lemma 4.1. Assume that $a_{\mathrm{per}}$ and $\phi$ satisfy (2.3), (2.4), (2.5) and (2.6). Assume furthermore the independence conditions (3.3) and (3.5). For any $0 \leq \alpha \leq \beta \leq 1$, and any $\mathcal{A} \in L^{\infty}(\alpha, \beta)$ with $\mathcal{A}^{\prime} \in L^{2}(\alpha, \beta)$, define the random variable

$$
\bar{Z}_{\varepsilon}(\alpha, \beta, \omega)=\frac{1}{\sqrt{\varepsilon}} \int_{\alpha}^{\beta} \mathcal{A}(t) \psi\left(\phi^{-1}\left(\frac{t}{\varepsilon}, \omega\right)\right) \mathrm{d} t,
$$

where the function $\psi$ is defined by (3.1). For any $p \in \mathbb{N}^{\star}$, there exists a deterministic constant $C_{p}$ independent of $\mathcal{A}, \varepsilon, \alpha$ and $\beta$, such that

$$
\forall \varepsilon>0, \quad \mathbb{E}\left[\bar{Z}_{\varepsilon}(\alpha, \beta, \cdot)^{2 p}\right] \leq C_{p}\left[(\beta-\alpha)^{p}+\varepsilon^{(p-1) / 2}\right]\left[\|\mathcal{A}\|_{L^{\infty}(\alpha, \beta)}^{2 p}+(\beta-\alpha)^{p}\left\|\mathcal{A}^{\prime}\right\|_{L^{2}(\alpha, \beta)}^{2 p}\right] .
$$

The above result heuristically implies that the quantity $\int_{\alpha}^{\beta} \mathcal{A}(t) \psi\left(\phi^{-1}\left(\frac{t}{\varepsilon}, \omega\right)\right) \mathrm{d} t$ is of the order of $\sqrt{\varepsilon}$.

We will show below a convergence result for the random variables $\bar{Z}_{\varepsilon}(\alpha, \beta, \omega)$ (see Lem. 4.6 below). The boundedness result stated in the above lemma is however sufficient for now. Using it, we indeed prove the following theorem, which is a key ingredient to prove Theorem 3.2.

Theorem 4.2. Assume that $a_{\mathrm{per}}$ and $\phi$ satisfy (2.3), (2.4), (2.5) and (2.6). Assume furthermore the independence conditions (3.3) and (3.5). Let $u^{\varepsilon}$ be the solution to (2.10) and $u_{\star}$ be the solution to (2.11). Then

$$
u^{\varepsilon}(x, \omega)-u_{\star}(x)=\int_{0}^{1} K_{0}(x, t) \psi\left(\phi^{-1}\left(\frac{t}{\varepsilon}, \omega\right)\right) \mathrm{d} t+r_{\varepsilon}(x, \omega),
$$

where $K_{0}$ is defined by (3.7), $\psi$ is defined by (3.1), and there exists a deterministic constant $C$ independent of $\varepsilon$ such that, for any $\varepsilon>0$,

$$
\sup _{x \in[0,1]} \mathbb{E}\left|r_{\varepsilon}(x, \cdot)\right| \leq C \varepsilon \quad \text { and } \quad \mathbb{E}\left[\left\|r_{\varepsilon}\right\|_{L^{2}(0,1)}^{2}\right] \leq C \varepsilon^{2}
$$

In addition, for any $p \in \mathbb{N}^{\star}$, there exists a deterministic constant $C_{p}$ independent of $\varepsilon$ such that

$$
\forall \varepsilon \in(0,1), \quad \forall(x, y) \in(0,1)^{2}, \quad \mathbb{E}\left[\left|r_{\varepsilon}(x, \cdot)-r_{\varepsilon}(y, \cdot)\right|^{2 p}\right] \leq C_{p} \varepsilon^{2 p} \sqrt{(x-y)^{2 p}+\varepsilon^{p-1 / 2}}
$$

and

$$
\forall \varepsilon \in(0,1), \quad \forall(x, y) \in(0,1)^{2}, \quad \mathbb{E}\left[\left|r_{\varepsilon}(x, \cdot)-r_{\varepsilon}(y, \cdot)\right|^{2 p}\right] \leq C_{p} \varepsilon^{p}(x-y)^{2 p} .
$$

In view of Lemma 4.1, the first term of the right-hand side of (4.2) is of the order of $\sqrt{\varepsilon}$. The term $r_{\varepsilon}$, which is of the order of $\varepsilon$ in view of (4.3), is hence a higher-order term. The bounds (4.4) and (4.5) will be useful below to show that some random process is tight (see Sect. 4.2, Thm. 4.5). 
Using the same arguments, we show the following result:

Theorem 4.3. Assume that $a_{\mathrm{per}}$ and $\phi$ satisfy (2.3), (2.4), (2.5) and (2.6). Assume furthermore the independence conditions (3.3) and (3.5). Let $u^{\varepsilon}$ be the solution to (2.10), $u_{\star}$ be the solution to (2.11), and $w$ be the corrector, solution to $(2.12)$ with $w(0, \omega)=0$ a.s. Then

$$
\begin{aligned}
\frac{d}{\mathrm{~d} x}\left(u^{\varepsilon}(x, \omega)-u_{\star}(x)-\varepsilon w\left(\frac{x}{\varepsilon}, \omega\right) \frac{d u_{\star}}{\mathrm{d} x}(x)\right)= & a_{\text {per }}^{-1}\left(\phi^{-1}\left(\frac{x}{\varepsilon}, \omega\right)\right) \int_{0}^{1} K_{1}(t) \psi\left(\phi^{-1}\left(\frac{t}{\varepsilon}, \omega\right)\right) \mathrm{d} t \\
& +f(x) \int_{0}^{x} \psi\left(\phi^{-1}\left(\frac{t}{\varepsilon}, \omega\right)\right) \mathrm{d} t+\bar{r}_{\varepsilon}(x, \omega),
\end{aligned}
$$

where $\psi$ is defined by (3.1), $K_{1}$ is given by

$$
K_{1}(t)=a^{\star}\left(F(t)-\int_{0}^{1} F(s) \mathrm{d} s\right) \quad \text { with } \quad F(t)=\int_{0}^{t} f(s) \mathrm{d} s,
$$

and there exists a deterministic constant $C$ independent of $\varepsilon$ such that, for all $\varepsilon>0$,

$$
\mathbb{E}\left[\sup _{x \in[0,1]}\left|\bar{r}_{\varepsilon}(x, \cdot)\right|\right] \leq C \varepsilon \quad \text { and } \quad \mathbb{E}\left[\sup _{x \in[0,1]}\left|\bar{r}_{\varepsilon}(x, \cdot)\right|^{2}\right] \leq C \varepsilon^{2} .
$$

Again, in view of Lemma 4.1, the two first terms of the right-hand side of (4.6) are of the order of $\sqrt{\varepsilon}$. The term $\bar{r}_{\varepsilon}$, which is of the order of $\varepsilon$, is hence a higher-order term.

Remark 4.4. It is easy to deduce from (4.6), using Lemma 4.1 and (4.8), that there exists a deterministic constant $C$ independent of $\varepsilon$ such that

$$
\mathbb{E}\left[\left\|\frac{d}{\mathrm{~d} x}\left(u^{\varepsilon}(x, \omega)-u_{\star}(x)-\varepsilon w\left(\frac{x}{\varepsilon}, \omega\right) \frac{d u_{\star}}{\mathrm{d} x}(x)\right)\right\|_{L^{2}(0,1)}^{2}\right] \leq C \varepsilon .
$$

Likewise, we deduce from (4.2), using Lemma 4.1 and (4.3), that

$$
\mathbb{E}\left[\left\|u^{\varepsilon}(x, \omega)-u_{\star}(x)\right\|_{L^{2}(0,1)}^{2}\right] \leq C \varepsilon .
$$

Using the expression (4.28) below, we infer from (4.9) and (4.10) that

$$
\mathbb{E}\left[\left\|u^{\varepsilon}(x, \omega)-u_{\star}(x)-\varepsilon w\left(\frac{x}{\varepsilon}, \omega\right) \frac{d u_{\star}}{\mathrm{d} x}(x)\right\|_{H^{1}(0,1)}^{2}\right] \leq C \varepsilon .
$$

We recover (in the one-dimensional situation) a classical result of homogenization: the corrector $w$ allows to obtain a convergence result in the $H^{1}$ strong norm. We refer to Theorem 3 of [17] for a corresponding result in classical random homogenization (in the multidimensional setting).

The proof of Theorems 4.2 and 4.3 are direct consequences of Lemma 4.1 and of the analytical expression of $u^{\varepsilon}$ and $u_{\star}$.

Proof of Theorem 4.2. Introduce $F(x)=\int_{0}^{x} f(t) \mathrm{d} t$. The solution to (2.10) reads

$$
u^{\varepsilon}(x, \omega)=c^{\varepsilon}(\omega) \int_{0}^{x} \frac{1}{a_{\text {per }}\left(\phi^{-1}\left(\frac{t}{\varepsilon}, \omega\right)\right)} \mathrm{d} t-\int_{0}^{x} \frac{F(t)}{a_{\text {per }}\left(\phi^{-1}\left(\frac{t}{\varepsilon}, \omega\right)\right)} \mathrm{d} t,
$$


where

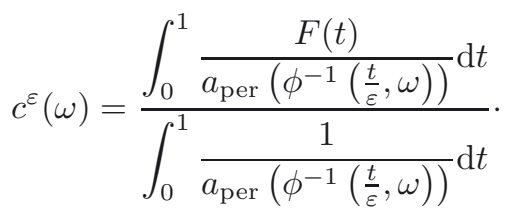

Likewise, the solution $u_{\star}$ of the homogenized problem (2.7) is

$$
u_{\star}(x)=c^{\star} \frac{x}{a^{\star}}-\int_{0}^{x} \frac{F(t)}{a^{\star}} \mathrm{d} t,
$$

where $a^{\star}$ is given by $(2.14)$ and

$$
c^{\star}=\int_{0}^{1} F(t) \mathrm{d} t
$$

Step 1. Representation formula

We compute the residual process using (4.14) and (4.12):

$$
\begin{aligned}
u^{\varepsilon}(x, \omega)-u_{\star}(x)= & c^{\varepsilon}(\omega) \int_{0}^{x} \frac{1}{a_{\text {per }}\left(\phi^{-1}\left(\frac{t}{\varepsilon}, \omega\right)\right)} \mathrm{d} t-c^{\star} \frac{x}{a^{\star}}-\int_{0}^{x} F(t) \psi\left(\phi^{-1}\left(\frac{t}{\varepsilon}, \omega\right)\right) \mathrm{d} t \\
= & c^{\varepsilon}(\omega) \int_{0}^{x} \psi\left(\phi^{-1}\left(\frac{t}{\varepsilon}, \omega\right)\right) \mathrm{d} t+\left(c^{\varepsilon}(\omega)-c^{\star}\right) \frac{x}{a^{\star}}-\int_{0}^{x} F(t) \psi\left(\phi^{-1}\left(\frac{t}{\varepsilon}, \omega\right)\right) \mathrm{d} t \\
= & \left(c^{\varepsilon}(\omega)-c^{\star}\right) \int_{0}^{x} \psi\left(\phi^{-1}\left(\frac{t}{\varepsilon}, \omega\right)\right) \mathrm{d} t+\left(c^{\varepsilon}(\omega)-c^{\star}\right) \frac{x}{a^{\star}} \\
& +\int_{0}^{x}\left(c^{\star}-F(t)\right) \psi\left(\phi^{-1}\left(\frac{t}{\varepsilon}, \omega\right)\right) \mathrm{d} t,
\end{aligned}
$$

where $\psi$ is defined by (3.1). We also infer from (4.13) that

$$
\begin{aligned}
c^{\varepsilon}(\omega)-c^{\star} & =\left(\int_{0}^{1} \frac{1}{a_{\text {per }}\left(\phi^{-1}\left(\frac{t}{\varepsilon}, \omega\right)\right)} \mathrm{d} t\right)^{-1} \int_{0}^{1}\left(F(t)-c^{\star}\right) \frac{1}{a_{\text {per }}\left(\phi^{-1}\left(\frac{t}{\varepsilon}, \omega\right)\right)} \mathrm{d} t \\
& =\left(\int_{0}^{1} \frac{1}{a_{\text {per }}\left(\phi^{-1}\left(\frac{t}{\varepsilon}, \omega\right)\right)} \mathrm{d} t\right)^{-1} \int_{0}^{1}\left(F(t)-c^{\star}\right) \psi\left(\phi^{-1}\left(\frac{t}{\varepsilon}, \omega\right)\right) \mathrm{d} t
\end{aligned}
$$

where we have used that, in view of (4.15), we have $\int_{0}^{1}\left(F(t)-c^{\star}\right) \frac{1}{a^{\star}} \mathrm{d} t=0$. Observe now that

$$
\left(\int_{0}^{1} \frac{1}{a_{\mathrm{per}}\left(\phi^{-1}\left(\frac{t}{\varepsilon}, \omega\right)\right)} \mathrm{d} t\right)^{-1}=a^{\star}-\frac{a^{\star}}{\int_{0}^{1} a_{\text {per }}^{-1}\left(\phi^{-1}\left(\frac{t}{\varepsilon}, \omega\right)\right) \mathrm{d} t} \int_{0}^{1} \psi\left(\phi^{-1}\left(\frac{t}{\varepsilon}, \omega\right)\right) \mathrm{d} t .
$$

We then deduce from (4.17) that

$$
c^{\varepsilon}(\omega)-c^{\star}=a^{\star} \int_{0}^{1}\left(F(t)-c^{\star}\right) \psi\left(\phi^{-1}\left(\frac{t}{\varepsilon}, \omega\right)\right) \mathrm{d} t-\rho^{\varepsilon}(\omega),
$$

where

$$
\rho^{\varepsilon}(\omega)=\left[\frac{a^{\star}}{\int_{0}^{1} a_{\text {per }}^{-1}\left(\phi^{-1}\left(\frac{t}{\varepsilon}, \omega\right)\right) \mathrm{d} t} \int_{0}^{1} \psi\left(\phi^{-1}\left(\frac{t}{\varepsilon}, \omega\right)\right) \mathrm{d} t\right] \int_{0}^{1}\left(F(t)-c^{\star}\right) \psi\left(\phi^{-1}\left(\frac{t}{\varepsilon}, \omega\right)\right) \mathrm{d} t .
$$


Collecting (4.16) and (4.18), we write

$$
\begin{aligned}
u^{\varepsilon}(x, \omega)-u_{\star}(x)= & \left(c^{\varepsilon}(\omega)-c^{\star}\right) \int_{0}^{x} \psi\left(\phi^{-1}\left(\frac{t}{\varepsilon}, \omega\right)\right) \mathrm{d} t+\int_{0}^{x}\left(c^{\star}-F(t)\right) \psi\left(\phi^{-1}\left(\frac{t}{\varepsilon}, \omega\right)\right) \mathrm{d} t \\
& +x \int_{0}^{1}\left(F(t)-c^{\star}\right) \psi\left(\phi^{-1}\left(\frac{t}{\varepsilon}, \omega\right)\right) \mathrm{d} t-\frac{x}{a^{\star}} \rho^{\varepsilon}(\omega) \\
= & r_{\varepsilon}(x, \omega)+\int_{0}^{x}\left(c^{\star}-F(t)\right) \psi\left(\phi^{-1}\left(\frac{t}{\varepsilon}, \omega\right)\right) \mathrm{d} t+x \int_{0}^{1}\left(F(t)-c^{\star}\right) \psi\left(\phi^{-1}\left(\frac{t}{\varepsilon}, \omega\right)\right) \mathrm{d} t \\
= & r_{\varepsilon}(x, \omega)+\int_{0}^{1} K_{0}(x, t) \psi\left(\phi^{-1}\left(\frac{t}{\varepsilon}, \omega\right)\right) \mathrm{d} t
\end{aligned}
$$

with

$$
K_{0}(x, t)=\left(\mathbf{1}_{[0, x]}(t)-x\right)\left(c^{\star}-F(t)\right)
$$

and

$$
r_{\varepsilon}(x, \omega)=-\frac{x}{a^{\star}} \rho^{\varepsilon}(\omega)+\left(c^{\varepsilon}(\omega)-c^{\star}\right) \int_{0}^{x} \psi\left(\phi^{-1}\left(\frac{t}{\varepsilon}, \omega\right)\right) \mathrm{d} t .
$$

In view of (4.15), we recover the expression (3.7) of $K_{0}$. We thus have written the residual in the form (4.2).

Step 2. Proof of the bound (4.3).

We first bound $\rho^{\varepsilon}(\omega)$. We infer from (4.19) that

$$
\left|\rho^{\varepsilon}(\omega)\right| \leq a^{+} a^{\star}\left|\int_{0}^{1} \psi\left(\phi^{-1}\left(\frac{t}{\varepsilon}, \omega\right)\right) \mathrm{d} t\right|\left|\int_{0}^{1}\left(F(t)-c^{\star}\right) \psi\left(\phi^{-1}\left(\frac{t}{\varepsilon}, \omega\right)\right) \mathrm{d} t\right| .
$$

Using the Cauchy-Schwartz inequality, we deduce that

$$
\mathbb{E}\left(\left|\rho^{\varepsilon}\right|\right) \leq \varepsilon a^{+} a^{\star} \sqrt{\mathbb{E}\left(\left|\frac{1}{\sqrt{\varepsilon}} \int_{0}^{1} \psi\left(\phi^{-1}\left(\frac{t}{\varepsilon}, \cdot\right)\right) \mathrm{d} t\right|^{2}\right)} \sqrt{\mathbb{E}\left(\left|\frac{1}{\sqrt{\varepsilon}} \int_{0}^{1}\left(F(t)-c^{\star}\right) \psi\left(\phi^{-1}\left(\frac{t}{\varepsilon}, \cdot\right)\right) \mathrm{d} t\right|^{2}\right)} .
$$

Using Lemma 4.1 with $p=1, \alpha=0, \beta=1, \mathcal{A}(t)=1$ and $\mathcal{A}(t)=F(t)-c^{\star}$, we obtain that there exists a constant $C$ independent of $\varepsilon$ such that

$$
\mathbb{E}\left(\left|\rho^{\varepsilon}\right|\right) \leq C \varepsilon
$$

We also deduce from (4.21) that, for any $p \in \mathbb{N}^{\star}$,

$$
\mathbb{E}\left(\left|\rho^{\varepsilon}\right|^{2 p}\right) \leq\left(a^{+} a^{\star}\right)^{2 p} \varepsilon^{2 p} \sqrt{\mathbb{E}\left(\left|\frac{1}{\sqrt{\varepsilon}} \int_{0}^{1} \psi\left(\phi^{-1}\left(\frac{t}{\varepsilon}, \cdot\right)\right) \mathrm{d} t\right|^{4 p}\right)} \sqrt{\mathbb{E}\left(\left|\frac{1}{\sqrt{\varepsilon}} \int_{0}^{1}\left(F(t)-c^{\star}\right) \psi\left(\phi^{-1}\left(\frac{t}{\varepsilon}, \cdot\right)\right) \mathrm{d} t\right|^{4 p}\right)} .
$$

Using again Lemma 4.1, we obtain that there exists a constant $C_{p}$ independent of $\varepsilon$ such that

$$
\mathbb{E}\left(\left|\rho^{\varepsilon}\right|^{2 p}\right) \leq C_{p} \varepsilon^{2 p} .
$$

Using the obtained bounds on $\rho^{\varepsilon}$, we now estimate $r_{\varepsilon}$. We infer from (4.18), using (4.23) and Lemma 4.1, that, for any $p \in \mathbb{N}^{\star}$,

$$
\begin{aligned}
\mathbb{E}\left(\left|c^{\varepsilon}-c^{\star}\right|^{2 p}\right) & \leq\left(a^{\star}\right)^{2 p} \varepsilon^{p} C_{p} \mathbb{E}\left(\left|\frac{1}{\sqrt{\varepsilon}} \int_{0}^{1}\left(F(t)-c^{\star}\right) \psi\left(\phi^{-1}\left(\frac{t}{\varepsilon}, \cdot\right)\right) \mathrm{d} t\right|^{2 p}\right)+C_{p} \mathbb{E}\left(\left|\rho^{\varepsilon}\right|^{2 p}\right) \\
& \leq\left(a^{\star}\right)^{2 p} C_{p} \varepsilon^{p}+C_{p} \varepsilon^{2 p} \\
& \leq C_{p} \varepsilon^{p}
\end{aligned}
$$


for a constant $C_{p}$ independent of $\varepsilon$. In view of (4.20), we thus obtain, using (4.22) and (4.24), that

$$
\begin{aligned}
\mathbb{E}\left(\left|r_{\varepsilon}(x, \cdot)\right|\right) & \leq \frac{x}{a^{\star}} \mathbb{E}\left(\left|\rho^{\varepsilon}\right|\right)+\mathbb{E}\left(\left|\left(c^{\varepsilon}-c^{\star}\right) \int_{0}^{x} \psi\left(\phi^{-1}\left(\frac{t}{\varepsilon}, \cdot\right)\right) \mathrm{d} t\right|\right) \\
& \leq C \varepsilon+\sqrt{\varepsilon} \sqrt{\mathbb{E}\left(\left|\left(c^{\varepsilon}-c^{\star}\right)\right|^{2}\right)} \sqrt{\mathbb{E}\left(\left|\frac{1}{\sqrt{\varepsilon}} \int_{0}^{x} \psi\left(\phi^{-1}\left(\frac{t}{\varepsilon}, \cdot\right)\right) \mathrm{d} t\right|^{2}\right)} \\
& \leq C \varepsilon
\end{aligned}
$$

for a constant $C$ independent from $\varepsilon$ and $x \in(0,1)$. This concludes the proof of the first assertion in (4.3).

Similarly, we have

$$
\left(r_{\varepsilon}(x, \omega)\right)^{2} \leq \frac{2}{\left(a^{\star}\right)^{2}} \rho^{\varepsilon}(\omega)^{2}+2\left(c^{\varepsilon}(\omega)-c^{\star}\right)^{2}\left|\int_{0}^{x} \psi\left(\phi^{-1}\left(\frac{t}{\varepsilon}, \omega\right)\right) \mathrm{d} t\right|^{2},
$$

thus

$$
\begin{aligned}
\mathbb{E}\left[\left\|r_{\varepsilon}\right\|_{L^{2}(0,1)}^{2}\right] & \leq \frac{2}{\left(a^{\star}\right)^{2}} \mathbb{E}\left[\left|\rho^{\varepsilon}\right|^{2}\right]+2 \int_{0}^{1} \mathbb{E}\left[\left(c^{\varepsilon}-c^{\star}\right)^{2}\left|\int_{0}^{x} \psi\left(\phi^{-1}\left(\frac{t}{\varepsilon}, \cdot\right)\right) \mathrm{d} t\right|^{2}\right] \mathrm{d} x \\
& \leq C \varepsilon^{2}+2 \int_{0}^{1} \sqrt{\mathbb{E}\left[\left(c^{\varepsilon}-c^{\star}\right)^{4}\right] \mathbb{E}\left[\left|\int_{0}^{x} \psi\left(\phi^{-1}\left(\frac{t}{\varepsilon}, \cdot\right)\right) \mathrm{d} t\right|^{4}\right]} \mathrm{d} x .
\end{aligned}
$$

Using (4.24) and Lemma 4.1 with $p=2$, we deduce that

$$
\mathbb{E}\left[\left\|r_{\varepsilon}\right\|_{L^{2}(0,1)}^{2}\right] \leq C \varepsilon^{2}+2 \int_{0}^{1} \sqrt{C \varepsilon^{4}} \mathrm{~d} x \leq C \varepsilon^{2}
$$

for a constant $C$ independent from $\varepsilon$. This concludes the proof of the second assertion in (4.3).

Step 3. Proof of the bounds (4.4) and (4.5).

We first prove (4.4). In view of (4.20), we have

$$
r_{\varepsilon}(x, \omega)-r_{\varepsilon}(y, \omega)=\frac{y-x}{a^{\star}} \rho^{\varepsilon}(\omega)+\left(c^{\varepsilon}(\omega)-c^{\star}\right) \int_{y}^{x} \psi\left(\phi^{-1}\left(\frac{t}{\varepsilon}, \omega\right)\right) \mathrm{d} t,
$$

thus

$$
\left|r_{\varepsilon}(x, \omega)-r_{\varepsilon}(y, \omega)\right|^{2 p} \leq C_{p}\left(\frac{y-x}{a^{\star}}\right)^{2 p}\left(\rho^{\varepsilon}(\omega)\right)^{2 p}+C_{p}\left(c^{\varepsilon}(\omega)-c^{\star}\right)^{2 p}\left|\int_{y}^{x} \psi\left(\phi^{-1}\left(\frac{t}{\varepsilon}, \omega\right)\right) \mathrm{d} t\right|^{2 p},
$$

and

$$
\begin{aligned}
\mathbb{E}\left[\left|r_{\varepsilon}(x, \cdot)-r_{\varepsilon}(y, \cdot)\right|^{2 p}\right] & \leq C_{p}\left(\frac{y-x}{a^{\star}}\right)^{2 p} \mathbb{E}\left[\left(\rho^{\varepsilon}\right)^{2 p}\right]+C_{p} \sqrt{\mathbb{E}\left[\left(c^{\varepsilon}-c^{\star}\right)^{4 p}\right]} \sqrt{\mathbb{E}\left[\left|\int_{y}^{x} \psi\left(\phi^{-1}\left(\frac{t}{\varepsilon}, \cdot\right)\right) \mathrm{d} t\right|^{4 p}\right]} \\
& \leq C_{p} \varepsilon^{2 p}(y-x)^{2 p}+C_{p} \sqrt{\varepsilon^{2 p}} \sqrt{\varepsilon^{2 p}\left((x-y)^{2 p}+\varepsilon^{(2 p-1) / 2}\right)} \\
& \leq C_{p} \varepsilon^{2 p}\left[(y-x)^{2 p}+\sqrt{(x-y)^{2 p}+\varepsilon^{p-1 / 2}}\right] .
\end{aligned}
$$


Since $|y-x| \leq 1$, we have $(y-x)^{2 p} \leq|y-x|^{p} \leq \sqrt{(x-y)^{2 p}+\varepsilon^{p-1 / 2}}$, and thus

$$
\mathbb{E}\left[\left|r_{\varepsilon}(x, \cdot)-r_{\varepsilon}(y, \cdot)\right|^{2 p}\right] \leq C_{p} \varepsilon^{2 p} \sqrt{(x-y)^{2 p}+\varepsilon^{p-1 / 2}} .
$$

This concludes the proof of (4.4). We now prove (4.5). We infer from (4.25) that

$$
\left|r_{\varepsilon}(x, \omega)-r_{\varepsilon}(y, \omega)\right|^{2 p} \leq C_{p}\left(\frac{y-x}{a^{\star}}\right)^{2 p}\left(\rho^{\varepsilon}(\omega)\right)^{2 p}+C_{p}\left(c^{\varepsilon}(\omega)-c^{\star}\right)^{2 p}(x-y)^{2 p}\|\psi\|_{L^{\infty}(\mathbb{R})}^{2 p},
$$

hence, using (4.23) and (4.24),

$$
\mathbb{E}\left[\left|r_{\varepsilon}(x, \cdot)-r_{\varepsilon}(y, \cdot)\right|^{2 p}\right] \leq C_{p}(x-y)^{2 p}\left[\varepsilon^{2 p}+\varepsilon^{p}\right] .
$$

This concludes the proof of (4.5) and thus that of Theorem 4.2.

Proof of Theorem 4.3. Recall that the solution to the corrector problem (2.12) satisfies (2.13). We thus have, using (4.12) and (4.14),

$$
\begin{aligned}
\frac{d}{\mathrm{~d} x}\left(u^{\varepsilon}(x, \omega)-u_{\star}(x)-\varepsilon w\left(\frac{x}{\varepsilon}, \omega\right) \frac{d u_{\star}}{\mathrm{d} x}(x)\right) & =\frac{d u^{\varepsilon}}{\mathrm{d} x}(x, \omega)-\frac{d u_{\star}}{\mathrm{d} x}(x)\left(1+w^{\prime}\left(\frac{x}{\varepsilon}, \omega\right)\right)-\varepsilon \frac{d^{2} u_{\star}}{\mathrm{d} x^{2}}(x) w\left(\frac{x}{\varepsilon}, \omega\right) \\
& =\frac{c^{\varepsilon}(\omega)-c^{\star}}{a_{\text {per }}\left(\phi^{-1}\left(\frac{x}{\varepsilon}, \omega\right)\right)}-\varepsilon \frac{d^{2} u_{\star}}{\mathrm{d} x^{2}}(x) w\left(\frac{x}{\varepsilon}, \omega\right) .
\end{aligned}
$$

Using (4.18), we deduce that

$$
\begin{aligned}
& \frac{d}{\mathrm{~d} x}\left(u^{\varepsilon}(x, \omega)-u_{\star}(x)-\varepsilon w\left(\frac{x}{\varepsilon}, \omega\right) \frac{d u_{\star}}{\mathrm{d} x}(x)\right) \\
= & \frac{a^{\star}}{a_{\text {per }}\left(\phi^{-1}\left(\frac{x}{\varepsilon}, \omega\right)\right)} \int_{0}^{1}\left(F(t)-c^{\star}\right) \psi\left(\phi^{-1}\left(\frac{t}{\varepsilon}, \omega\right)\right) \mathrm{d} t-\varepsilon \frac{d^{2} u_{\star}}{\mathrm{d} x^{2}}(x) w\left(\frac{x}{\varepsilon}, \omega\right)-\frac{\rho^{\varepsilon}(\omega)}{a_{\text {per }}\left(\phi^{-1}\left(\frac{x}{\varepsilon}, \omega\right)\right)} \\
= & a_{\text {per }}^{-1}\left(\phi^{-1}\left(\frac{x}{\varepsilon}, \omega\right)\right) \int_{0}^{1} K_{1}(t) \psi\left(\phi^{-1}\left(\frac{t}{\varepsilon}, \omega\right)\right) \mathrm{d} t+\varepsilon \frac{f(x)}{a^{\star}} w\left(\frac{x}{\varepsilon}, \omega\right)+\bar{r}^{\varepsilon}(x, \omega),
\end{aligned}
$$

with $K_{1}$ defined by $(4.7)$ and

$$
\bar{r}^{\varepsilon}(x, \omega)=-\frac{\rho^{\varepsilon}(\omega)}{a_{\text {per }}\left(\phi^{-1}\left(\frac{x}{\varepsilon}, \omega\right)\right)} .
$$

Observe now that, in view of (2.13) and (3.1), we have

$$
w(y, \omega)=a^{\star} \int_{0}^{y} \psi\left(\phi^{-1}(t, \omega)\right) \mathrm{d} t,
$$

where we have chosen the integration constant in $w$ such that $w(0, \omega)=0$ almost surely. Thus

$$
w\left(\frac{x}{\varepsilon}, \omega\right)=a^{\star} \int_{0}^{x / \varepsilon} \psi\left(\phi^{-1}(t, \omega)\right) \mathrm{d} t=\frac{a^{\star}}{\varepsilon} \int_{0}^{x} \psi\left(\phi^{-1}\left(\frac{t}{\varepsilon}, \omega\right)\right) \mathrm{d} t .
$$

Collecting this equation with (4.26) yields (4.6). The bound (4.8) follows from (4.27), (4.22) and (4.23). This concludes the proof of Theorem 4.3. 


\subsection{Proof of Theorem 3.2}

In this section, we prove that the random process $\frac{u^{\varepsilon}(x, \omega)-u_{\star}(x)}{\sqrt{\varepsilon}}$ converges in distribution to a Gaussian random process that we characterize. Using (4.2), we see that

$$
\frac{u^{\varepsilon}(x, \omega)-u_{\star}(x)}{\sqrt{\varepsilon}}=G_{\varepsilon}(x, \omega)+R_{\varepsilon}(x, \omega),
$$

where

$$
\begin{aligned}
G_{\varepsilon}(x, \omega) & =\frac{1}{\sqrt{\varepsilon}} \int_{0}^{1} K_{0}(x, t) \psi\left(\phi^{-1}\left(\frac{t}{\varepsilon}, \omega\right)\right) \mathrm{d} t \\
R_{\varepsilon}(x, \omega) & =\frac{1}{\sqrt{\varepsilon}} r_{\varepsilon}(x, \omega) .
\end{aligned}
$$

In view of (4.3), we have

$$
\sup _{x \in[0,1]} \mathbb{E}\left|R_{\varepsilon}(x, \cdot)\right| \leq C \sqrt{\varepsilon}
$$

for a constant $C$ independent of $\varepsilon$. As a consequence,

$$
\forall x \in(0,1), \quad R_{\varepsilon}(x, \cdot) \text { converges to } 0 \text { in probability. }
$$

We are thus left with studying the behaviour of $G_{\varepsilon}(x, \omega)$ as $\varepsilon \rightarrow 0$.

To prove that the random process $G_{\varepsilon}(x, \omega)$ converges in distribution, we will use the following result:

Theorem 4.5 ([6], p. 54). Suppose that $\left(G_{\varepsilon}\right)_{\varepsilon \in(0,1)}$ and $G_{0}$ are random processes with values in the space of continuous functions $C^{0}(0,1)$ with $G_{\varepsilon}(0, \omega)=G_{0}(0, \omega)=0$ almost surely. Assume that

(i) for any $k \in \mathbb{N}^{\star}$ and any $0 \leq x_{1} \leq \ldots \leq x_{k} \leq 1$, the random variable $\left(G_{\varepsilon}\left(x_{1}, \omega\right), \ldots, G_{\varepsilon}\left(x_{k}, \omega\right)\right) \in \mathbb{R}^{k}$ converges in distribution to the random variable $\left(G_{0}\left(x_{1}, \omega\right), \ldots, G_{0}\left(x_{k}, \omega\right)\right)$ as $\varepsilon \rightarrow 0$.

(ii) $\left(G_{\varepsilon}\right)_{\varepsilon \in(0,1)}$ is a tight sequence of random processes in $C^{0}(0,1)$. A sufficient condition for the tightness of $\left(G_{\varepsilon}\right)_{\varepsilon \in(0,1)}$ is the Kolmogorov criterion: there exist $\delta>0, \beta>0$ and $C>0$ such that

$$
\forall \varepsilon \in(0,1), \quad \forall(x, y) \in(0,1)^{2}, \quad \mathbb{E}\left[\left|G_{\varepsilon}(x, \cdot)-G_{\varepsilon}(y, \cdot)\right|^{\beta}\right] \leq C|x-y|^{1+\delta} .
$$

Then the process $G_{\varepsilon}$ converges in distribution to the process $G_{0}$ as $\varepsilon$ goes to 0.

For any $x \in(0,1)$, the random variable $G_{\varepsilon}(x, \omega)$ is of the form of the random variable $\bar{Z}_{\varepsilon}(\alpha, \beta, \omega)$ defined in (4.1), with $\alpha=0, \beta=1$ and $\mathcal{A}(t)=K_{0}(x, t)$. In Lemma 4.1, we have shown that the random variable $\bar{Z}_{\varepsilon}(\alpha, \beta, \omega)$ is bounded in the $L^{2 p}$ norm. We now show that this random variable converges in law to a Gaussian random variable. This will be a key ingredient to prove the first condition of Theorem 4.5.

Lemma 4.6. Assume that $a_{\mathrm{per}}$ and $\phi$ satisfy (2.3), (2.4), (2.5) and (2.6). Assume furthermore the independence conditions (3.3) and (3.5). For any $0 \leq \alpha \leq \beta \leq 1$, consider a function $\mathcal{A}$, piecewise continuous over $(\alpha, \beta)$, with a finite number of discontinuities located at points $\left\{t_{k}\right\}_{1 \leq k \leq m}$, and such that $\mathcal{A}^{\prime} \in L^{1}\left(t_{k}, t_{k+1}\right)$ for any $1 \leq k \leq m-1$. Consider the random variable

$$
\bar{Z}_{\varepsilon}(\alpha, \beta, \omega)=\frac{1}{\sqrt{\varepsilon}} \int_{\alpha}^{\beta} \mathcal{A}(t) \psi\left(\phi^{-1}\left(\frac{t}{\varepsilon}, \omega\right)\right) \mathrm{d} t
$$


where the function $\psi$ is defined by (3.1). Then $\bar{Z}_{\varepsilon}(\alpha, \beta, \omega)$ converges in distribution to a Gaussian random variable $\bar{Z}_{0}(\alpha, \beta, \omega)$, of mean zero and variance $\bar{\sigma}(\alpha, \beta)=\frac{\mathbb{V} a r\left(Y_{0}\right)}{\mathbb{E}\left(\int_{0}^{1} \phi^{\prime}\right)}\|\mathcal{A}\|_{L^{2}(\alpha, \beta)}^{2}$, with $\operatorname{Var}\left(Y_{0}\right)=\mathbb{E}\left[\left(\int_{0}^{1} \psi \phi^{\prime}\right)^{2}\right]$. We write

$$
\bar{Z}_{0}(\alpha, \beta, \omega)=\frac{\sqrt{\mathbb{V a r}\left(Y_{0}\right)}}{\sqrt{\mathbb{E}\left(\int_{0}^{1} \phi^{\prime}\right)}} \int_{\alpha}^{\beta} \mathcal{A}(t) \mathrm{d} W_{t},
$$

where $W_{t}$ denote the classical Brownian motion.

The proof of Lemma 4.6 is postponed until Section 5.2.

To prove the second condition of Theorem 4.5, we will show that $G_{\varepsilon}(x, \omega)$ satisfies (4.33). Observe that

$$
G_{\varepsilon}(x, \omega)=\frac{1}{\sqrt{\varepsilon}} \int_{0}^{x} \mathcal{A}(t) \psi\left(\phi^{-1}\left(\frac{t}{\varepsilon}, \omega\right)\right) \mathrm{d} t-\frac{x}{\sqrt{\varepsilon}} \int_{0}^{1} \mathcal{A}(t) \psi\left(\phi^{-1}\left(\frac{t}{\varepsilon}, \omega\right)\right) \mathrm{d} t
$$

with $\mathcal{A}(t)=\int_{0}^{1} F(s) \mathrm{d} s-F(t)$, where $F(t)=\int_{0}^{t} f(s) \mathrm{d} s$. To prove that $G_{\varepsilon}(x, \omega)$ satisfies (4.33), we will use the following result, the proof of which is postponed until Section 5.3.

Lemma 4.7. Assume that $a_{\text {per }}$ and $\phi$ satisfy (2.3), (2.4), (2.5) and (2.6). Assume furthermore the independence conditions (3.3) and (3.5). Consider two functions $\mathcal{A}_{1}$ and $\mathcal{A}_{2}$ with $\mathcal{A}_{j} \in L^{\infty}(0,1)$ and $\mathcal{A}_{j}^{\prime} \in L^{2}(0,1), j=1,2$. For any $x \in(0,1)$, consider the random variable

$$
H_{\varepsilon}(x, \omega)=\frac{1}{\sqrt{\varepsilon}} \int_{0}^{x} \mathcal{A}_{1}(t) \psi\left(\phi^{-1}\left(\frac{t}{\varepsilon}, \omega\right)\right) \mathrm{d} t+\frac{x}{\sqrt{\varepsilon}} \int_{0}^{1} \mathcal{A}_{2}(t) \psi\left(\phi^{-1}\left(\frac{t}{\varepsilon}, \omega\right)\right) \mathrm{d} t
$$

where the function $\psi$ is defined by (3.1).

For any $p \in \mathbb{N}^{\star}$, there exists a deterministic constant $C_{p}$ independent of $\varepsilon, x$ and $y$ such that

$$
\forall \varepsilon \in(0,1), \quad \forall(x, y) \in(0,1)^{2}, \quad \mathbb{E}\left[\left|H_{\varepsilon}(x, \cdot)-H_{\varepsilon}(y, \cdot)\right|^{2 p}\right] \leq C_{p}\left(|x-y|^{p}+\varepsilon^{(p-1) / 2}\right) .
$$

In addition, there exists a deterministic constant $C$ independent of $\varepsilon, x$ and $y$ such that, for any $x$ and $y$ with $|x-y| \leq \varepsilon$,

$$
\left|H_{\varepsilon}(x, \omega)-H_{\varepsilon}(y, \omega)\right| \leq C \sqrt{|x-y|} \quad \text { a.s. }
$$

We are now in position to prove Theorem 3.2.

Proof of Theorem 3.2. We have seen (see (4.29)) that

$$
\frac{u^{\varepsilon}(x, \omega)-u_{\star}(x)}{\sqrt{\varepsilon}}=G_{\varepsilon}(x, \omega)+R_{\varepsilon}(x, \omega),
$$

where $G_{\varepsilon}(x, \omega)$ and $R_{\varepsilon}(x, \omega)$ are defined by (4.30) and (4.31), respectively.

Let us study the process $G_{\varepsilon}(x, \omega)$, which reads, we recall,

$$
G_{\varepsilon}(x, \omega)=\frac{1}{\sqrt{\varepsilon}} \int_{0}^{1} K_{0}(x, t) \psi\left(\phi^{-1}\left(\frac{t}{\varepsilon}, \omega\right)\right) \mathrm{d} t .
$$

As $K_{0}(0, t)=0$ for any $t$, we have that $G_{\varepsilon}(0, \omega)=0$ for any $\varepsilon$, almost surely. We first show that this process satisfies the first condition of Theorem 4.5. For each set of points $0 \leq x_{1} \leq \ldots \leq x_{k} \leq 1$ and each $\mathcal{X}=$ $\left(\xi_{1}, \ldots, \xi_{k}\right) \in \mathbb{R}^{k}$, we consider the random variable

$$
z_{\varepsilon}(\omega)=\sum_{j=1}^{k} \xi_{j} G_{\varepsilon}\left(x_{j}, \omega\right)
$$


Observing that

$$
G_{\varepsilon}\left(x_{j}, \omega\right)=\frac{1}{\sqrt{\varepsilon}} \int_{0}^{1} K_{0}\left(x_{j}, t\right) \psi\left(\phi^{-1}\left(\frac{t}{\varepsilon}, \omega\right)\right) \mathrm{d} t
$$

we can write $z_{\varepsilon}$ as

$$
z_{\varepsilon}(\omega)=\frac{1}{\sqrt{\varepsilon}} \int_{0}^{1} \mathcal{A}_{\mathcal{X}}(t) \psi\left(\phi^{-1}\left(\frac{t}{\varepsilon}, \omega\right)\right) \mathrm{d} t
$$

where

$$
\mathcal{A}_{\mathcal{X}}(t)=\sum_{j=1}^{k} \xi_{j} K_{0}\left(x_{j}, t\right)=\left(\int_{0}^{1} F(s) \mathrm{d} s-F(t)\right) \sum_{j=1}^{k} \xi_{j}\left(\mathbf{1}_{\left[0, x_{j}\right]}(t)-x_{j}\right),
$$

with $F(t)=\int_{0}^{t} f(s) \mathrm{d} s$. By assumption, $f \in L^{2}(0,1)$, thus $\mathcal{A}_{\mathcal{X}}$ is piecewise continuous with a finite number of discontinuities located at $\left\{x_{j}\right\}_{1 \leq j \leq k}$. In addition, we see that, over each $\left(x_{i}, x_{i+1}\right), 1 \leq i \leq k-1$,

$$
\mathcal{A}_{\mathcal{X}}^{\prime}(t)=\left(\sum_{j=1}^{k} \xi_{j} x_{j}-\sum_{j>i}^{k} \xi_{j}\right) f(t)
$$

is in $L^{2}\left(x_{i}, x_{i+1}\right) \subset L^{1}\left(x_{i}, x_{i+1}\right)$. Thus, using Lemma 4.6, we obtain that $z_{\varepsilon}(\omega)$ converges in law to

$$
z_{0}(\omega)=\sum_{j=1}^{k} \xi_{j} G_{0}\left(x_{j}, \omega\right)
$$

where $G_{0}$ is defined by (3.6). This implies that

$$
\lim _{\varepsilon \rightarrow 0} \mathbb{E}\left[\exp \left(i \sum_{j=1}^{k} \xi_{j} G_{\varepsilon}\left(x_{j}, \cdot\right)\right)\right]=\lim _{\varepsilon \rightarrow 0} \mathbb{E}\left(\exp \left(i z_{\varepsilon}\right)\right)=\mathbb{E}\left(\exp \left(i z_{0}\right)\right)=\mathbb{E}\left[\exp \left(i \sum_{j=1}^{k} \xi_{j} G_{0}\left(x_{j}, \cdot\right)\right)\right] .
$$

Hence, for any $k \in \mathbb{N}^{\star}$ and any $0 \leq x_{1} \leq \ldots \leq x_{k} \leq 1$,

$$
\left(G_{\varepsilon}\left(x_{1}, \omega\right), \ldots, G_{\varepsilon}\left(x_{k}, \omega\right)\right) \text { converges in distribution to }\left(G_{0}\left(x_{1}, \omega\right), \ldots, G_{0}\left(x_{k}, \omega\right)\right) \text { as } \varepsilon \rightarrow 0 .
$$

Collecting (4.40), (4.41) and (4.32), we obtain that

$$
\begin{aligned}
& \text { the residual process } \frac{u^{\varepsilon}(x, \omega)-u_{\star}(x)}{\sqrt{\varepsilon}} \text { satisfies Condition (i) of Theorem } 4.5 \\
& \text { with the limit process } G_{0}(x, \omega) \text { defined by }(3.6) \text {. }
\end{aligned}
$$

We now prove the Kolmogorov criterion, first on the random process $G_{\varepsilon}(x, \omega)$, next on the process $\frac{u^{\varepsilon}-u_{\star}}{\sqrt{\varepsilon}}$. This will show Condition (ii) of Theorem 4.5. Following (4.36), we write

$$
G_{\varepsilon}(x, \omega)=\frac{1}{\sqrt{\varepsilon}} \int_{0}^{x} \mathcal{A}(t) \psi\left(\phi^{-1}\left(\frac{t}{\varepsilon}, \omega\right)\right) \mathrm{d} t-\frac{x}{\sqrt{\varepsilon}} \int_{0}^{1} \mathcal{A}(t) \psi\left(\phi^{-1}\left(\frac{t}{\varepsilon}, \omega\right)\right) \mathrm{d} t
$$

with $\mathcal{A}(t)=\int_{0}^{1} F(s) \mathrm{d} s-F(t)$, where $F(t)=\int_{0}^{t} f(s) \mathrm{d} s$. The assumptions of Lemma 4.7 are satisfied, thus, for any $p \in \mathbb{N}^{\star}$, there exists $C_{p}$ such that

$$
\forall \varepsilon \in(0,1), \quad \forall(x, y) \in(0,1)^{2}, \quad \mathbb{E}\left[\left|G_{\varepsilon}(x, \cdot)-G_{\varepsilon}(y, \cdot)\right|^{2 p}\right] \leq C_{p}\left(|x-y|^{p}+\varepsilon^{(p-1) / 2}\right) .
$$


This directly implies that

$$
\text { when }|x-y| \geq \varepsilon, \quad \mathbb{E}\left[\left|G_{\varepsilon}(x, \cdot)-G_{\varepsilon}(y, \cdot)\right|^{2 p}\right] \leq C_{p}|x-y|^{(p-1) / 2} .
$$

When $|x-y| \leq \varepsilon$, using (4.39), we see that there exists a deterministic constant $C$ independent of $\varepsilon, x$ and $y$ such that

$$
\mathbb{E}\left[\left|G_{\varepsilon}(x, \cdot)-G_{\varepsilon}(y, \cdot)\right|^{2 p}\right] \leq C|x-y|^{p} \leq C|x-y|^{(p-1) / 2} \quad \text { when } \quad|x-y| \leq \varepsilon .
$$

Collecting (4.44) and (4.45), we obtain that

$$
\forall \varepsilon \in(0,1), \quad \forall(x, y) \in(0,1)^{2}, \quad \mathbb{E}\left[\left|G_{\varepsilon}(x, \cdot)-G_{\varepsilon}(y, \cdot)\right|^{2 p}\right] \leq C|x-y|^{(p-1) / 2} .
$$

We now turn to the process $R_{\varepsilon}(x, \omega)$. In view of (4.31) and (4.4), there exists $C_{p}$ such that

$$
\forall \varepsilon \in(0,1), \quad \forall(x, y) \in(0,1)^{2}, \quad \mathbb{E}\left[\left|R_{\varepsilon}(x, \cdot)-R_{\varepsilon}(y, \cdot)\right|^{2 p}\right] \leq C_{p} \varepsilon^{p} \sqrt{(x-y)^{2 p}+\varepsilon^{p-1 / 2}} .
$$

Hence, we deduce that

$$
\mathbb{E}\left[\left|R_{\varepsilon}(x, \cdot)-R_{\varepsilon}(y, \cdot)\right|^{2 p}\right] \leq C_{p} \varepsilon^{p}|x-y|^{(2 p-1) / 4} \quad \text { when } \quad|x-y| \geq \varepsilon
$$

When $|x-y| \leq \varepsilon$, using (4.5), we see that

$$
\mathbb{E}\left[\left|R_{\varepsilon}(x, \cdot)-R_{\varepsilon}(y, \cdot)\right|^{2 p}\right] \leq C_{p}(x-y)^{2 p} \leq C_{p} \varepsilon^{p}|x-y|^{p} \leq C_{p} \varepsilon^{p}|x-y|^{(2 p-1) / 4} \quad \text { when } \quad|x-y| \leq \varepsilon .
$$

Collecting (4.48) and (4.49), we obtain that

$$
\forall \varepsilon \in(0,1), \quad \forall(x, y) \in(0,1)^{2}, \quad \mathbb{E}\left[\left|R_{\varepsilon}(x, \cdot)-R_{\varepsilon}(y, \cdot)\right|^{2 p}\right] \leq C \varepsilon^{p}|x-y|^{(2 p-1) / 4} .
$$

We next write, using (4.40),

$$
\left|\frac{u^{\varepsilon}(x, \omega)-u_{\star}(x)}{\sqrt{\varepsilon}}-\frac{u^{\varepsilon}(y, \omega)-u_{\star}(y)}{\sqrt{\varepsilon}}\right|^{2 p} \leq C_{p}\left|G_{\varepsilon}(x, \omega)-G_{\varepsilon}(y, \omega)\right|^{2 p}+C_{p}\left|R_{\varepsilon}(x, \omega)-R_{\varepsilon}(y, \omega)\right|^{2 p} .
$$

Collecting (4.46) and (4.50), we obtain that

$$
\forall \varepsilon \in(0,1), \quad \forall(x, y) \in(0,1)^{2}, \quad \mathbb{E}\left[\left|\frac{u^{\varepsilon}(x, \cdot)-u_{\star}(x)}{\sqrt{\varepsilon}}-\frac{u^{\varepsilon}(y, \cdot)-u_{\star}(y)}{\sqrt{\varepsilon}}\right|^{2 p}\right] \leq C|x-y|^{(p-1) / 2}\left(1+\varepsilon^{p}\right) .
$$

We thus obtain that

$$
\begin{aligned}
& \text { the residual process } \frac{u^{\varepsilon}(x, \omega)-u_{\star}(x)}{\sqrt{\varepsilon}} \text { satisfies Condition (ii) of Theorem } 4.5 \\
& \text { with the exponents } \beta=2 p \text { and } \delta=p / 2-3 / 2 \text {. }
\end{aligned}
$$

Choosing $p$ such that $\beta>0$ and $\delta>0$ (it suffices to choose $p>3$ ), and collecting (4.42) and (4.53), we see that the random process $\frac{u^{\varepsilon}(x, \omega)-u_{\star}(x)}{\sqrt{\varepsilon}}$ satisfies the assumptions of Theorem 4.5. It thus converges in law to the Gaussian process $G_{0}(x, \omega)$ defined by (3.6). This concludes the proof of Theorem 3.2. 


\section{TECHNICAL PROOFS}

We collect here the proofs of Lemmas 4.1, 4.6 and 4.7 .

\subsection{Proof of Lemma 4.1}

Lemma 4.1 is a consequence of the following result:

Lemma 5.1. Assume that $a_{\text {per }}$ and $\phi$ satisfy (2.3), (2.4), (2.5) and (2.6). Assume furthermore the independence conditions (3.3) and (3.5). For any $0 \leq \alpha \leq \beta \leq 1$, define the random variable

$$
Z_{\varepsilon}(\alpha, \beta, \omega)=\frac{1}{\sqrt{\varepsilon}} \int_{\alpha}^{\beta} \psi\left(\phi^{-1}\left(\frac{t}{\varepsilon}, \omega\right)\right) \mathrm{d} t,
$$

where the function $\psi$ is defined by (3.1). For any $p \in \mathbb{N}^{\star}$, there exists a deterministic constant $C_{p}$ independent of $\varepsilon, \alpha$ and $\beta$ such that

$$
\forall \varepsilon>0, \quad \mathbb{E}\left[Z_{\varepsilon}(\alpha, \beta, \cdot)^{2 p}\right] \leq C_{p}\left[(\beta-\alpha)^{p}+\varepsilon^{(p-1) / 2}\right] .
$$

We first prove Lemma 5.1, and next Lemma 4.1.

Proof of Lemma 5.1. Using the variable $s=\phi^{-1}\left(\frac{t}{\varepsilon}, \omega\right)$, we write

$$
Z_{\varepsilon}(\alpha, \beta, \omega)=\frac{1}{\sqrt{\varepsilon}} \int_{\alpha}^{\beta} \psi\left(\phi^{-1}\left(\frac{t}{\varepsilon}, \omega\right)\right) \mathrm{d} t=\sqrt{\varepsilon} \int_{\phi^{-1}(\alpha / \varepsilon, \omega)}^{\phi^{-1}(\beta / \varepsilon, \omega)} \psi(s) \phi^{\prime}(s, \omega) \mathrm{d} s .
$$

For future use, we introduce, for any $x \in(0,1)$, the notation

$$
\bar{K}_{x}(\omega)=\left\lfloor\phi^{-1}(x / \varepsilon, \omega)\right\rfloor .
$$

In view of (2.4) and (2.5), we have

$$
M^{-1}\left|\frac{\beta-\alpha}{\varepsilon}\right| \leq\left|\phi^{-1}\left(\frac{\alpha}{\varepsilon}, \omega\right)-\phi^{-1}\left(\frac{\beta}{\varepsilon}, \omega\right)\right| \leq \nu^{-1}\left|\frac{\beta-\alpha}{\varepsilon}\right| .
$$

Hence, up to some boundary terms (due to the fact that $\phi^{-1}(\alpha / \varepsilon, \omega)$ and $\phi^{-1}(\beta / \varepsilon, \omega)$ are not integer numbers), $Z_{\varepsilon} / \sqrt{\varepsilon}$ is a sum of the variables $Y_{k}$ defined by $(3.2)$, with a number of terms of the order of $\varepsilon^{-1}$. Note however that this number of terms, equal to $\bar{K}_{\beta}(\omega)-\bar{K}_{\alpha}(\omega)$, is random. To proceed, we write $Z_{\varepsilon}$ as the sum of two contributions: (i) a sum of the variables $Y_{k}$ with a deterministic number of terms, and (ii) a remainder, that will be successively estimated.

Following (5.2), we have

$$
\begin{aligned}
Z_{\varepsilon}(\alpha, \beta, \omega) & =\sqrt{\varepsilon}\left(\int_{\frac{\alpha}{\varepsilon \mathbb{E}\left(\int_{0}^{1} \phi^{\prime}\right)}}^{\frac{\beta}{\varepsilon \mathbb{(}\left(\int_{0}^{1} \phi^{\prime}\right)}} \psi(t) \phi^{\prime}(t, \omega) \mathrm{d} t+\int_{\frac{\beta}{\varepsilon \mathbb{E}\left(\int_{0}^{1} \phi^{\prime}\right)}}^{\phi^{-1}(\beta / \varepsilon, \omega)} \psi(t) \phi^{\prime}(t, \omega) \mathrm{d} t+\int_{\phi^{-1}(\alpha / \varepsilon, \omega)}^{\frac{\alpha}{\varepsilon \mathbb{E}\left(\int_{0}^{1} \phi^{\prime}\right)}} \psi(t) \phi^{\prime}(t, \omega) \mathrm{d} t\right) \\
& =B_{\varepsilon}(\alpha, \beta, \omega)+A_{\varepsilon}(\beta, \omega)-A_{\varepsilon}(\alpha, \omega)
\end{aligned}
$$

with

$$
\begin{aligned}
A_{\varepsilon}(x, \omega) & =\sqrt{\varepsilon} \int_{\frac{x}{\varepsilon \mathbb{E}\left(\int_{0}^{1} \phi^{\prime}\right)}}^{\phi^{-1}(x / \varepsilon, \omega)} \psi(t) \phi^{\prime}(t, \omega) \mathrm{d} t, \\
B_{\varepsilon}(\alpha, \beta, \omega) & =\sqrt{\varepsilon} \int_{\frac{\alpha}{\varepsilon \mathbb{E}\left(\int_{0}^{1} \phi^{\prime}\right)}}^{\frac{\beta}{\varepsilon \mathbb{E}\left(\int_{0}^{1} \phi^{\prime}\right)}} \psi(t) \phi^{\prime}(t, \omega) \mathrm{d} t .
\end{aligned}
$$


Note that, up to boundary terms, $B_{\varepsilon}(\alpha, \beta, \omega) / \sqrt{\varepsilon}$ is a sum of the variables $Y_{k}$, with a deterministic number of terms. We infer from (5.3) that, for any $p \in \mathbb{N}^{\star}$,

$$
\mathbb{E}\left[Z_{\varepsilon}(\alpha, \beta, \cdot)^{2 p}\right] \leq C_{p} \mathbb{E}\left[B_{\varepsilon}(\alpha, \beta, \cdot)^{2 p}\right]+C_{p} \mathbb{E}\left[A_{\varepsilon}(\alpha, \cdot)^{2 p}\right]+C_{p} \mathbb{E}\left[A_{\varepsilon}(\beta, \cdot)^{2 p}\right]
$$

where the constant $C_{p}$ only depends on $p$. We now estimate $B_{\varepsilon}$, and next $A_{\varepsilon}$.

Step 1. Estimation of $B_{\varepsilon}$.

$$
\begin{aligned}
\text { Denoting by } K_{\alpha} & =\left\lfloor\frac{\alpha}{\varepsilon \mathbb{E}\left(\int_{0}^{1} \phi^{\prime}\right)}\right\rfloor \text { and } K_{\beta}=\left\lfloor\frac{\beta}{\varepsilon \mathbb{E}\left(\int_{0}^{1} \phi^{\prime}\right)}\right\rfloor \text {, we have } \\
B_{\varepsilon}(\alpha, \beta, \omega) & =\sqrt{\varepsilon}\left(\sum_{k=1+K_{\alpha}}^{K_{\beta}-1} \int_{k}^{k+1} \psi(t) \phi^{\prime}(t, \omega) \mathrm{d} t+\int_{K_{\beta}}^{\frac{\beta}{\varepsilon \mathbb{E}\left(\int_{0}^{1} \phi^{\prime}\right)}} \psi(t) \phi^{\prime}(t, \omega) \mathrm{d} t+\int_{\frac{\alpha}{\varepsilon \mathbb{E}\left(\int_{0}^{1} \phi^{\prime}\right)}}^{1+K_{\alpha}} \psi(t) \phi^{\prime}(t, \omega) \mathrm{d} t\right) \\
& =\sqrt{\varepsilon} \sum_{k=1+K_{\alpha}}^{K_{\beta}-1} Y_{k}(\omega)+\sqrt{\varepsilon} \int_{K_{\beta}}^{\frac{\beta}{\varepsilon \mathbb{E}\left(\int_{0}^{1} \phi^{\prime}\right)}} \psi(t) \phi^{\prime}(t, \omega) \mathrm{d} t+\sqrt{\varepsilon} \int_{\frac{\alpha}{\varepsilon \mathbb{E}\left(\int_{0}^{1} \phi^{\prime}\right)}}^{1+K_{\alpha}} \psi(t) \phi^{\prime}(t, \omega) \mathrm{d} t
\end{aligned}
$$

where we recall that $Y_{k}$ is defined by (3.2). We thus obtain, for a deterministic constant $C_{p}$ that only depends on $p$,

$$
\left|B_{\varepsilon}(\alpha, \beta, \omega)\right|^{2 p} \leq C_{p} \varepsilon^{p}\left|\sum_{k=1+K_{\alpha}}^{K_{\beta}-1} Y_{k}(\omega)\right|^{2 p}+C_{p} \varepsilon^{p}\|\psi\|_{L^{\infty}(\mathbb{R})}^{2 p}\left\|\phi^{\prime}\right\|_{L^{\infty}(\mathbb{R} \times \Omega)}^{2 p} .
$$

Recall that $\left(Y_{k}\right)_{k \in \mathbb{Z}}$ is a sequence of independent identically distributed variables, with $\mathbb{E}\left(Y_{k}\right)=0$. We now use the fact that any such variables satisfy the following bounds:

$$
\forall p \in \mathbb{N}^{\star}, \quad \exists C_{p}>0, \quad \forall N \in \mathbb{N}^{\star}, \quad\left|\mathbb{E}\left[\left(\frac{1}{N} \sum_{k=1}^{N} Y_{k}\right)^{2 p}\right]\right| \leq \frac{C_{p}}{N^{p}}
$$

for a constant $C_{p}$ that depends on $p$ and the moments of $Y_{k}$, up to order $2 p$. This is proved by developing the power $2 p$ of the sum, and then using the fact that the variables are i.i.d and have mean value zero. In our case, the variables $Y_{k}$ are bounded almost surely, and thus all their moments are finite. We thus deduce from (5.8) and (5.9) that

$$
\begin{aligned}
\mathbb{E}\left[B_{\varepsilon}(\alpha, \beta, \cdot)^{2 p}\right] & \leq C_{p} \varepsilon^{p} \mathbb{E}\left[\left|\sum_{k=1+K_{\alpha}}^{K_{\beta}-1} Y_{k}\right|^{2 p}\right]+C_{p} \varepsilon^{p}\|\psi\|_{L^{\infty}(\mathbb{R})}^{2 p}\left\|\phi^{\prime}\right\|_{L^{\infty}(\mathbb{R} \times \Omega)}^{2 p} \\
& \leq C_{p} \varepsilon^{p}\left(K_{\beta}-K_{\alpha}-1\right)^{p}+C_{p} \varepsilon^{p}\|\psi\|_{L^{\infty}(\mathbb{R})}^{2 p}\left\|\phi^{\prime}\right\|_{L^{\infty}(\mathbb{R} \times \Omega)}^{2 p} \\
& \leq C_{p}(\beta-\alpha)^{p}+C_{p} \varepsilon^{p}\|\psi\|_{L^{\infty}(\mathbb{R})}^{2 p}\left\|\phi^{\prime}\right\|_{L^{\infty}(\mathbb{R} \times \Omega)}^{2 p} .
\end{aligned}
$$

Step 2. Estimation of $A_{\varepsilon}$.

We now bound $A_{\varepsilon}(x, \omega)$, for any $x \in[0,1]$. Denoting $K_{x}=\left\lfloor\frac{x}{\varepsilon \mathbb{E}\left(\int_{0}^{1} \phi^{\prime}\right)}\right\rfloor$ and $\bar{K}_{x}(\omega)=\left\lfloor\phi^{-1}(x / \varepsilon, \omega)\right\rfloor$, we have

$$
A_{\varepsilon}(x, \omega)=\sqrt{\varepsilon}\left(\int_{K_{x}}^{\bar{K}_{x}(\omega)} \psi(t) \phi^{\prime}(t, \omega) \mathrm{d} t+\int_{\frac{x}{\varepsilon \mathbb{E}\left(\int_{0}^{1} \phi^{\prime}\right)}}^{K_{x}} \psi(t) \phi^{\prime}(t, \omega) \mathrm{d} t+\int_{\bar{K}_{x}(\omega)}^{\phi^{-1}(x / \varepsilon, \omega)} \psi(t) \phi^{\prime}(t, \omega) \mathrm{d} t\right)
$$


hence

$$
\left|A_{\varepsilon}(x, \omega)\right| \leq \sqrt{\varepsilon}\left|\int_{K_{x}}^{\bar{K}_{x}(\omega)} \psi(t) \phi^{\prime}(t, \omega) \mathrm{d} t\right|+2 \sqrt{\varepsilon}\|\psi\|_{L^{\infty}(\mathbb{R})}\left\|\phi^{\prime}\right\|_{L^{\infty}(\mathbb{R} \times \Omega)},
$$

thus

$$
\mathbb{E}\left[A_{\varepsilon}(x, \cdot)^{2 p}\right] \leq C_{p} \varepsilon^{p} \mathbb{E}\left[\left|\int_{K_{x}}^{\bar{K}_{x}} \psi(t) \phi^{\prime}(t, \cdot) \mathrm{d} t\right|^{2 p}\right]+C_{p} \varepsilon^{p}\|\psi\|_{L^{\infty}(\mathbb{R})}^{2 p}\left\|\phi^{\prime}\right\|_{L^{\infty}(\mathbb{R} \times \Omega)}^{2 p} .
$$

Let us now bound the first term of the above right-hand side. The difficulty stems from the fact that the random variable $\bar{K}_{x}(\omega)$ is not independent from the random process $\phi^{\prime}(t, \omega)$. We write, using the bound (5.9) and Young's inequality with parameter $\frac{\gamma}{j^{2 p+2}}>0$ (where $\gamma>0$ is arbitrary), that

$$
\begin{aligned}
\mathbb{E}\left[\left|\int_{K_{x}}^{\bar{K}_{x}} \psi(t) \phi^{\prime}(t, \cdot) \mathrm{d} t\right|^{2 p}\right] & =\sum_{j \in \mathbb{Z}^{\star}} \mathbb{E}\left[\left|\int_{K_{x}}^{\bar{K}_{x}} \psi(t) \phi^{\prime}(t, \cdot) \mathrm{d} t\right|^{2 p} \mathbf{1}_{\bar{K}_{x}(\omega)=K_{x}+j}\right] \\
& \leq \sum_{j \in \mathbb{Z}^{\star}} \frac{\gamma}{2 j^{2 p+2}} \mathbb{E}\left[\left|\int_{K_{x}}^{K_{x}+j} \psi(t) \phi^{\prime}(t, \cdot) \mathrm{d} t\right|^{4 p}\right]+\frac{j^{2 p+2}}{2 \gamma} \mathbb{P}\left[\bar{K}_{x}(\omega)=K_{x}+j\right] \\
& \leq \sum_{j \in \mathbb{Z}^{\star}} \frac{\gamma}{2 j^{2 p+2}} \mathbb{E}\left[\left|\sum_{k=K_{x}}^{K_{x}+j-1} Y_{k}\right|^{4 p}\right]+\frac{j^{2 p+2}}{2 \gamma} \mathbb{P}\left[\bar{K}_{x}(\omega)=K_{x}+j\right] \\
& \leq \sum_{j \in \mathbb{Z}^{\star}} C_{2 p} \frac{\gamma}{2 j^{2}}+\frac{j^{2 p+2}}{2 \gamma} \mathbb{P}\left[\bar{K}_{x}(\omega)=K_{x}+j\right] \\
& \leq C_{2 p} \frac{\gamma}{2}+\frac{1}{2 \gamma} \mathbb{E}\left[\left|\bar{K}_{x}-K_{x}\right|^{2 p+2}\right] .
\end{aligned}
$$

We are now left with bounding from above $\mathbb{E}\left(\left|\bar{K}_{x}-K_{x}\right|^{2 p+2}\right)$. To this aim, we first bound from above $\left|\bar{K}_{x}(\omega)-K_{x}\right|^{2 p+2}$ :

$$
\begin{aligned}
\left|\bar{K}_{x}(\omega)-K_{x}\right|^{2 p+2} & \leq C_{p}\left(\left|K_{x}-\phi^{-1}(x / \varepsilon, \omega)\right|^{2 p+2}+\left|\phi^{-1}(x / \varepsilon, \omega)-\bar{K}_{x}(\omega)\right|^{2 p+2}\right) \\
& \leq C_{p}\left(\left|K_{x}-\phi^{-1}(x / \varepsilon, \omega)\right|^{2 p+2}+1\right) .
\end{aligned}
$$

Recall now that, in view of (2.4), we have $|a-b| \leq \nu^{-1}|\phi(a, \omega)-\phi(b, \omega)|$ for any $a$ and $b$, almost surely. We get

$$
\left|\bar{K}_{x}(\omega)-K_{x}\right|^{2 p+2} \leq \frac{C_{p}}{\nu^{2 p+2}}\left(\left|\phi\left(K_{x}, \omega\right)-\frac{x}{\varepsilon}\right|^{2 p+2}+\nu^{2 p+2}\right)
$$

We now recall that the random variables $D_{k}(\omega)=\int_{k}^{k+1} \phi^{\prime}(t, \omega) \mathrm{d} t$, introduced in (3.4), are assumed to be i.i.d. random variables. Writing $\phi(x, \omega)=\phi(0, \omega)+\int_{0}^{x} \phi^{\prime}(t, \omega) \mathrm{d} t$, we obtain that

$$
\begin{aligned}
\left|\phi\left(K_{x}, \omega\right)-\frac{x}{\varepsilon}\right|^{2 p+2} & \leq C_{p}\left(\left|\int_{0}^{K_{x}} \phi^{\prime}(t, \omega) \mathrm{d} t-K_{x} \mathbb{E}\left(D_{0}\right)\right|^{2 p+2}+|\phi(0, \omega)|^{2 p+2}+\left|K_{x} \mathbb{E}\left(D_{0}\right)-\frac{x}{\varepsilon}\right|^{2 p+2}\right) \\
& \leq C_{p}\left(\left|\sum_{k=0}^{K_{x}-1}\left(D_{k}(\omega)-\mathbb{E}\left(D_{0}\right)\right)\right|^{2 p+2}+|\phi(0, \omega)|^{2 p+2}+\left|K_{x} \mathbb{E}\left(D_{0}\right)-\frac{x}{\varepsilon}\right|^{2 p+2}\right),
\end{aligned}
$$


where, we recall, $K_{x}=\left\lfloor\frac{x}{\varepsilon \mathbb{E}\left(\int_{0}^{1} \phi^{\prime}\right)}\right\rfloor$. Observing that $\mathbb{E}\left(D_{0}\right)=\mathbb{E}\left(\int_{0}^{1} \phi^{\prime}\right)$, we have $\left|\frac{x}{\varepsilon}-K_{x} \mathbb{E}\left(D_{0}\right)\right| \leq \mathbb{E}\left(D_{0}\right)$, thus

$$
\left|\phi\left(K_{x}, \omega\right)-\frac{x}{\varepsilon}\right|^{2 p+2} \leq C_{p}\left(\left|\sum_{k=0}^{K_{x}-1}\left(D_{k}(\omega)-\mathbb{E}\left(D_{0}\right)\right)\right|^{2 p+2}+|\phi(0, \omega)|^{2 p+2}+\left|\mathbb{E}\left(D_{0}\right)\right|^{2 p+2}\right) .
$$

Collecting (5.13) and (5.14), we obtain

$$
\left|\bar{K}_{x}(\omega)-K_{x}\right|^{2 p+2} \leq \frac{C_{p}}{\nu^{2 p+2}}\left(\left|\sum_{k=0}^{K_{x}-1}\left(D_{k}(\omega)-\mathbb{E}\left(D_{0}\right)\right)\right|^{2 p+2}+|\phi(0, \omega)|^{2 p+2}+\left|\mathbb{E}\left(D_{0}\right)\right|^{2 p+2}+\nu^{2 p+2}\right) .
$$

Next, we take the expectation of the above inequality and use (5.9) to get

$$
\mathbb{E}\left(\left|\bar{K}_{x}-K_{x}\right|^{2 p+2}\right) \leq \frac{C_{p}}{\nu^{2 p+2}}\left(K_{x}^{p+1}+\mathbb{E}\left(|\phi(0, \cdot)|^{2 p+2}\right)+\left|\mathbb{E}\left(D_{0}\right)\right|^{2 p+2}+\nu^{2 p+2}\right) .
$$

Since $K_{x}=\left\lfloor\frac{x}{\varepsilon \mathbb{E}\left(\int_{0}^{1} \phi^{\prime}\right)}\right\rfloor$, we know that $K_{x}$ is of the order of $1 / \varepsilon$, and thus

$$
\forall x \in(0,1), \quad \mathbb{E}\left(\left|\bar{K}_{x}(\omega)-K_{x}\right|^{2 p+2}\right) \leq C_{p} \frac{1}{\varepsilon^{p+1}}
$$

for a constant $C_{p}$ independent of $\varepsilon$ and $x$. We infer from (5.12) and (5.15) that

$$
\forall \gamma>0, \quad \mathbb{E}\left[\left|\int_{K_{x}}^{\bar{K}_{x}} \psi(t) \phi^{\prime}(t, \cdot) \mathrm{d} t\right|^{2 p}\right] \leq C_{p}\left(\frac{\gamma}{2}+\frac{1}{2 \gamma \varepsilon^{p+1}}\right)
$$

Taking $\gamma^{-1}=\varepsilon^{(p+1) / 2}$ leads to

$$
\mathbb{E}\left[\left|\int_{K_{x}}^{\bar{K}_{x}(\omega)} \psi(t) \phi^{\prime}(t, \omega) \mathrm{d} t\right|^{2 p}\right] \leq C_{p} \frac{1}{\varepsilon^{(p+1) / 2}} .
$$

Collecting (5.11) and (5.16), we obtain

$$
\forall x \in(0,1), \quad \mathbb{E}\left[A_{\varepsilon}(x, \cdot)^{2 p}\right] \leq C_{p} \varepsilon^{(p-1) / 2}
$$

for a constant $C_{p}$ independent of $\varepsilon$ and $x$.

Step 3. Conclusion

Collecting (5.6), (5.10) and (5.17) (which is legitimate since $0 \leq \alpha \leq \beta \leq 1$ ), we obtain

$$
\mathbb{E}\left[Z_{\varepsilon}(\alpha, \beta, \cdot)^{2 p}\right] \leq C_{p}\left[(\beta-\alpha)^{p}+\varepsilon^{p}+\varepsilon^{(p-1) / 2}\right] \leq C_{p}\left[(\beta-\alpha)^{p}+\varepsilon^{(p-1) / 2}\right]
$$

where $C_{p}$ is a deterministic constant independent from $\alpha, \beta$ and $\varepsilon$. This concludes the proof of Lemma 5.1. 
Proof of Lemma 4.1. The result directly follows from Lemma 5.1 and an integration by part argument. We consider the random variable $Z_{\varepsilon}(\alpha, \beta, \omega)$ defined by

$$
Z_{\varepsilon}(\alpha, \beta, \omega)=\frac{1}{\sqrt{\varepsilon}} \int_{\alpha}^{\beta} \psi\left(\phi^{-1}\left(\frac{t}{\varepsilon}, \omega\right)\right) \mathrm{d} t .
$$

Integrating by part, we see that

$$
\bar{Z}_{\varepsilon}(\alpha, \beta, \omega)=\left[\mathcal{A}(t) Z_{\varepsilon}(\alpha, t, \omega)\right]_{\alpha}^{\beta}-\int_{\alpha}^{\beta} \mathcal{A}^{\prime}(t) Z_{\varepsilon}(\alpha, t, \omega) \mathrm{d} t=\mathcal{A}(\beta) Z_{\varepsilon}(\alpha, \beta, \omega)-\int_{\alpha}^{\beta} \mathcal{A}^{\prime}(t) Z_{\varepsilon}(\alpha, t, \omega) \mathrm{d} t .
$$

Using the Cauchy-Schwartz inequality, we obtain

$$
\bar{Z}_{\varepsilon}(\alpha, \beta, \omega)^{2} \leq 2 \mathcal{A}(\beta)^{2} Z_{\varepsilon}(\alpha, \beta, \omega)^{2}+2 \int_{\alpha}^{\beta}\left(\mathcal{A}^{\prime}(t)\right)^{2} \mathrm{~d} t \int_{\alpha}^{\beta} Z_{\varepsilon}(\alpha, t, \omega)^{2} \mathrm{~d} t
$$

We now take the power $p$ of this estimate:

$$
\begin{aligned}
\bar{Z}_{\varepsilon}(\alpha, \beta, \omega)^{2 p} & \leq C_{p}\|\mathcal{A}\|_{L^{\infty}(\alpha, \beta)}^{2 p} Z_{\varepsilon}(\alpha, \beta, \omega)^{2 p}+C_{p}\left\|\mathcal{A}^{\prime}\right\|_{L^{2}(\alpha, \beta)}^{2 p}\left(\int_{\alpha}^{\beta} Z_{\varepsilon}(\alpha, t, \omega)^{2} \mathrm{~d} t\right)^{p} \\
& \leq C_{p}\|\mathcal{A}\|_{L^{\infty}(\alpha, \beta)}^{2 p} Z_{\varepsilon}(\alpha, \beta, \omega)^{2 p}+C_{p}\left\|\mathcal{A}^{\prime}\right\|_{L^{2}(\alpha, \beta)}^{2 p} \int_{\alpha}^{\beta} Z_{\varepsilon}(\alpha, t, \omega)^{2 p} \mathrm{~d} t\left(\int_{\alpha}^{\beta} \mathrm{d} t\right)^{p / q} \\
& \leq C_{p}\|\mathcal{A}\|_{L^{\infty}(\alpha, \beta)}^{2 p} Z_{\varepsilon}(\alpha, \beta, \omega)^{2 p}+C_{p}(\beta-\alpha)^{p-1}\left\|\mathcal{A}^{\prime}\right\|_{L^{2}(\alpha, \beta)}^{2 p} \int_{\alpha}^{\beta} Z_{\varepsilon}(\alpha, t, \omega)^{2 p} \mathrm{~d} t
\end{aligned}
$$

where we have used Hölder inequality with $1=1 / p+1 / q$. Using Lemma 5.1, we thus obtain

$$
\begin{aligned}
& \mathbb{E}\left[\bar{Z}_{\varepsilon}(\alpha, \beta, \cdot)^{2 p}\right] \\
\leq & C_{p}\|\mathcal{A}\|_{L^{\infty}(\alpha, \beta)}^{2 p} \mathbb{E}\left[Z_{\varepsilon}(\alpha, \beta, \cdot)^{2 p}\right]+C_{p}(\beta-\alpha)^{p-1}\left\|\mathcal{A}^{\prime}\right\|_{L^{2}(\alpha, \beta)}^{2 p} \int_{\alpha}^{\beta} \mathbb{E}\left[Z_{\varepsilon}(\alpha, t, \cdot)^{2 p}\right] \mathrm{d} t \\
\leq & C_{p}\|\mathcal{A}\|_{L^{\infty}(\alpha, \beta)}^{2 p}\left[(\beta-\alpha)^{p}+\varepsilon^{(p-1) / 2}\right]+C_{p}(\beta-\alpha)^{p-1}\left\|\mathcal{A}^{\prime}\right\|_{L^{2}(\alpha, \beta)}^{2 p} \int_{\alpha}^{\beta}\left[(t-\alpha)^{p}+\varepsilon^{(p-1) / 2}\right] \mathrm{d} t \\
\leq & C_{p}\|\mathcal{A}\|_{L^{\infty}(\alpha, \beta)}^{2 p}\left[(\beta-\alpha)^{p}+\varepsilon^{(p-1) / 2}\right]+C_{p}(\beta-\alpha)^{p-1}\left\|\mathcal{A}^{\prime}\right\|_{L^{2}(\alpha, \beta)}^{2 p}\left[(\beta-\alpha)^{p+1}+(\beta-\alpha) \varepsilon^{(p-1) / 2}\right] \\
\leq & C_{p}\left[(\beta-\alpha)^{p}+\varepsilon^{(p-1) / 2}\right]\left[\|\mathcal{A}\|_{L^{\infty}(\alpha, \beta)}^{2 p}+(\beta-\alpha)^{p}\left\|\mathcal{A}^{\prime}\right\|_{L^{2}(\alpha, \beta)}^{2 p}\right] .
\end{aligned}
$$

This concludes the proof of Lemma 4.1.

\subsection{Proof of Lemma 4.6}

By definition,

$$
\bar{Z}_{\varepsilon}(\alpha, \beta, \omega)=\frac{1}{\sqrt{\varepsilon}} \int_{\alpha}^{\beta} \mathcal{A}(t) \psi\left(\phi^{-1}\left(\frac{t}{\varepsilon}, \omega\right)\right) \mathrm{d} t .
$$

We start by replacing the function $\mathcal{A}$ by a piecewise constant function $\widetilde{\mathcal{A}}$, that we will choose later as an accurate approximation of $\mathcal{A}$, in a sense to be made precise. We thus introduce the function $\widetilde{\mathcal{A}}$ defined by

$$
\widetilde{\mathcal{A}}(t)=\sum_{p=1}^{N} \mathcal{A}_{p} \mathbf{1}_{\left(t_{p}, t_{p+1}\right)}(t),
$$


with $\alpha=t_{1}<t_{2}<\ldots<t_{N+1}=\beta$. Hence the sets $\left(t_{p}, t_{p+1}\right)$ are disjoint one from another, and $\cup_{1 \leq p \leq N}\left[t_{p}, t_{p+1}\right]=[\alpha, \beta]$. We associate to this function $\widetilde{\mathcal{A}}$ the random variable

$$
\widetilde{Z}_{\varepsilon}(\alpha, \beta, \omega)=\frac{1}{\sqrt{\varepsilon}} \int_{\alpha}^{\beta} \widetilde{\mathcal{A}}(t) \psi\left(\phi^{-1}\left(\frac{t}{\varepsilon}, \omega\right)\right) \mathrm{d} t=\frac{1}{\sqrt{\varepsilon}} \sum_{p=1}^{N} \mathcal{A}_{p} \int_{t_{p}}^{t_{p+1}} \psi\left(\phi^{-1}\left(\frac{t}{\varepsilon}, \omega\right)\right) \mathrm{d} t .
$$

Step 1. $\widetilde{Z}_{\varepsilon}(\alpha, \beta, \omega)$ converges in law to a Gaussian random variable.

In view of (5.1) and (5.3), we have, for each $p$,

$$
\frac{1}{\sqrt{\varepsilon}} \int_{t_{p}}^{t_{p+1}} \psi\left(\phi^{-1}\left(\frac{t}{\varepsilon}, \omega\right)\right) \mathrm{d} t=Z_{\varepsilon}\left(t_{p}, t_{p+1}, \omega\right)=B_{\varepsilon}\left(t_{p}, t_{p+1}, \omega\right)+A_{\varepsilon}\left(t_{p+1}, \omega\right)-A_{\varepsilon}\left(t_{p}, \omega\right) .
$$

We can write $B_{\varepsilon}($ see $(5.7))$ as

$$
B_{\varepsilon}\left(t_{p}, t_{p+1}, \omega\right)=\widetilde{B}_{\varepsilon}\left(t_{p}, t_{p+1}, \omega\right)+\widetilde{R}_{\varepsilon, p}(\omega),
$$

where

$$
\widetilde{B}_{\varepsilon}\left(t_{p}, t_{p+1}, \omega\right)=\sqrt{\varepsilon} \sum_{k=1+K_{p}}^{K_{p+1}-1} Y_{k}(\omega)
$$

with $K_{p}=\left\lfloor\frac{t_{p}}{\varepsilon \mathbb{E}\left(\int_{0}^{1} \phi^{\prime}\right)}\right\rfloor$, and

$$
\widetilde{R}_{\varepsilon, p}(\omega)=\sqrt{\varepsilon} \int_{K_{p+1}}^{\frac{t_{p+1}}{\varepsilon \mathbb{E}\left(\int_{0}^{1} \phi^{\prime}\right)}} \psi(t) \phi^{\prime}(t, \omega) \mathrm{d} t+\sqrt{\varepsilon} \int_{\frac{t_{p}}{\varepsilon \mathbb{E}\left(\int_{0}^{1} \phi^{\prime}\right)}}^{1+K_{p}} \psi(t) \phi^{\prime}(t, \omega) \mathrm{d} t
$$

We hence write

$$
\widetilde{Z}_{\varepsilon}(\alpha, \beta, \omega)=\sum_{p=1}^{N} \mathcal{A}_{p}\left(\widetilde{B}_{\varepsilon}\left(t_{p}, t_{p+1}, \omega\right)+\widetilde{R}_{\varepsilon, p}(\omega)+A_{\varepsilon}\left(t_{p+1}, \omega\right)-A_{\varepsilon}\left(t_{p}, \omega\right)\right) .
$$

We successively study $\widetilde{R}_{\varepsilon, p}, A_{\varepsilon}$ and $\widetilde{B}_{\varepsilon}$.

Observe first that $\widetilde{R}_{\varepsilon, p}$ satisfies

$$
\left|\widetilde{R}_{\varepsilon, p}(\omega)\right| \leq 2 \sqrt{\varepsilon}\|\psi\|_{L^{\infty}(\mathbb{R})}\left\|\phi^{\prime}\right\|_{L^{\infty}(\mathbb{R} \times \Omega)},
$$

and hence goes to 0 as $\varepsilon \rightarrow 0$ almost surely.

We now turn to $A_{\varepsilon}$. For any $x \in[0,1]$ and any $\lambda>0$, we have, using the Chebyshev inequality and (5.17), that

$$
\mathbb{P}\left(\left|A_{\varepsilon}(x, \cdot)\right|>\lambda\right) \leq \frac{\mathbb{E}\left[\left|A_{\varepsilon}(x, \cdot)\right|^{4}\right]}{\lambda^{4}} \leq C_{2} \frac{\varepsilon^{1 / 2}}{\lambda^{4}}
$$

where the constant $C_{2}$ is independent of $\varepsilon$ and $x$. We hence see that, for any $\lambda>0$, we have

$$
\lim _{\varepsilon \rightarrow 0} \mathbb{P}\left(\left|A_{\varepsilon}(x, \cdot)\right|>\lambda\right)=0,
$$

i.e. $A_{\varepsilon}(x, \omega)$ converges in probability to 0 as $\varepsilon \rightarrow 0$, for any $x$.

We eventually turn to $\widetilde{B}_{\varepsilon}$. Recall that $\left(Y_{k}(\omega)\right)_{k \in \mathbb{Z}}$ is a sequence of i.i.d. variables of mean zero (see assumption (3.3)). Using the Central Limit Theorem, we obtain that $\widetilde{B}_{\varepsilon}\left(t_{p}, t_{p+1}, \omega\right)$ defined by $(5.20)$ converges in law to a Gaussian variable,

$$
\widetilde{B}_{\varepsilon}\left(t_{p}, t_{p+1}, \omega\right) \underset{\varepsilon \rightarrow 0}{\stackrel{\mathcal{L}}{\longrightarrow}} \mathcal{N}\left(0, \sigma_{p}\right),
$$


the variance of which is

$$
\sigma_{p}=\frac{t_{p+1}-t_{p}}{\mathbb{E}\left(\int_{0}^{1} \phi^{\prime}\right)} \operatorname{Var}\left(Y_{0}\right)
$$

In addition, the random variables $\widetilde{B}_{\varepsilon}\left(t_{p}, t_{p+1}, \omega\right)$ are independent one from another.

We are now in position to prove the convergence of $\widetilde{Z}_{\varepsilon}$. Since $\widetilde{R}_{\varepsilon}(\omega)$ and $A_{\varepsilon}(x, \omega)$ converge to zero in probability for any $x$, and the random variables $\widetilde{B}_{\varepsilon}\left(t_{p}, t_{p+1}, \omega\right)$ converge in law for any $p$ and are independent one from another, we deduce from $(5.21)$ that $\widetilde{Z}_{\varepsilon}(\alpha, \beta, \omega)$ converges in law to a Gaussian variable,

$$
\widetilde{Z}_{\varepsilon}(\alpha, \beta, \omega) \underset{\varepsilon \rightarrow 0}{\stackrel{\mathcal{L}}{\longrightarrow}} \widetilde{Z}_{0}(\alpha, \beta, \omega) \sim \mathcal{N}(0, \widetilde{\sigma}(\alpha, \beta))
$$

the variance of which is

$$
\widetilde{\sigma}(\alpha, \beta)=\sum_{p=1}^{N} \mathcal{A}_{p}^{2} \sigma_{p}=\sum_{p=1}^{N} \mathcal{A}_{p}^{2} \frac{t_{p+1}-t_{p}}{\mathbb{E}\left(\int_{0}^{1} \phi^{\prime}\right)} \operatorname{Var}\left(Y_{0}\right)=\frac{\operatorname{Var}\left(Y_{0}\right)}{\mathbb{E}\left(\int_{0}^{1} \phi^{\prime}\right)}\|\widetilde{\mathcal{A}}\|_{L^{2}(\alpha, \beta)}^{2} .
$$

Step 2. Convergence of the random variable $\bar{Z}_{\varepsilon}(\alpha, \beta, \omega)$.

Recall that $\mathcal{A}$ is piecewise continuous with a finite number of discontinuities located at $\left\{t_{k}\right\}_{1 \leq k \leq m}$ and that, for each $1 \leq k \leq m-1, \mathcal{A}^{\prime} \in L^{1}\left(t_{k}, t_{k+1}\right)$. Introduce the broken $L^{1}$-norm of $\mathcal{A}^{\prime}$ :

$$
\left|\mathcal{A}^{\prime}\right|_{L^{1}(\alpha, \beta)}:=\left\|\mathcal{A}^{\prime}\right\|_{L^{1}\left(\alpha, t_{1}\right)}+\sum_{k=1}^{m-1}\left\|\mathcal{A}^{\prime}\right\|_{L^{1}\left(t_{k}, t_{k+1}\right)}+\left\|\mathcal{A}^{\prime}\right\|_{L^{1}\left(t_{m}, \beta\right)} .
$$

Let us fix some $\eta>0$, and let us complement the previous set of points $\left(t_{p}\right)_{1 \leq p \leq N+1}$ such that

$$
\alpha=t_{1}, t_{N+1}=\beta \text { and } 0<t_{p+1}-t_{p} \leq \eta \text { for any } p .
$$

We set

$$
\mathcal{A}_{p}=\mathcal{A}\left(t_{p+1}^{-}\right)
$$

and consider the function $\widetilde{\mathcal{A}}$ and the random variable $\widetilde{Z}_{\varepsilon}(\alpha, \beta, \omega)$ defined by (5.18) and (5.19).

We write, for any $\xi \in \mathbb{R}$,

$$
\begin{aligned}
\mathbb{E}\left(\mathrm{e}^{i \xi \bar{Z}_{\varepsilon}(\alpha, \beta, \cdot)}\right)-\mathbb{E}\left(\mathrm{e}^{i \xi \bar{Z}_{0}(\alpha, \beta, \cdot)}\right)= & \mathbb{E}\left(\mathrm{e}^{i \xi \bar{Z}_{\varepsilon}(\alpha, \beta, \cdot)}-\mathrm{e}^{i \xi \widetilde{Z}_{\varepsilon}(\alpha, \beta, \cdot)}\right)+\mathbb{E}\left(\mathrm{e}^{i \xi \widetilde{Z}_{\varepsilon}(\alpha, \beta, \cdot)}\right)-\mathbb{E}\left(\mathrm{e}^{i \xi \widetilde{Z}_{0}(\alpha, \beta, \cdot)}\right) \\
& +\mathbb{E}\left(\mathrm{e}^{i \xi \widetilde{Z}_{0}(\alpha, \beta, \cdot)}-\mathrm{e}^{i \xi \bar{Z}_{0}(\alpha, \beta, \cdot)}\right),
\end{aligned}
$$

where $\bar{Z}_{0}(\alpha, \beta, \omega)$ is a Gaussian random variable distributed according to $\mathcal{N}(0, \bar{\sigma}(\alpha, \beta))$, with the variance

$$
\bar{\sigma}(\alpha, \beta)=\frac{\operatorname{Var}\left(Y_{0}\right)}{\mathbb{E}\left(\int_{0}^{1} \phi^{\prime}\right)}\|\mathcal{A}\|_{L^{2}(\alpha, \beta)}^{2} .
$$

We successively estimate the three terms of the right-hand side of (5.24).

For the first term, we first see that

$$
\left|\mathbb{E}\left(\mathrm{e}^{i \xi \bar{Z}_{\varepsilon}(\alpha, \beta, \cdot)}-\mathrm{e}^{i \xi \widetilde{Z}_{\varepsilon}(\alpha, \beta, \cdot)}\right)\right| \leq \mathbb{E}\left|\mathrm{e}^{i \xi \bar{Z}_{\varepsilon}(\alpha, \beta, \cdot)}-\mathrm{e}^{i \xi \widetilde{Z}_{\varepsilon}(\alpha, \beta, \cdot)}\right| \leq|\xi| \mathbb{E}\left|\bar{Z}_{\varepsilon}(\alpha, \beta, \cdot)-\widetilde{Z}_{\varepsilon}(\alpha, \beta, \cdot)\right| \cdot
$$


We next compute

$$
\begin{aligned}
\bar{Z}_{\varepsilon}(\alpha, \beta, \omega)-\widetilde{Z}_{\varepsilon}(\alpha, \beta, \omega) & =\frac{1}{\sqrt{\varepsilon}} \int_{\alpha}^{\beta}(\mathcal{A}(t)-\widetilde{\mathcal{A}}(t)) \psi\left(\phi^{-1}\left(\frac{t}{\varepsilon}, \omega\right)\right) \mathrm{d} t \\
& =\sum_{p=1}^{N} \frac{1}{\sqrt{\varepsilon}} \int_{t_{p}}^{t_{p+1}}\left(\mathcal{A}(t)-\mathcal{A}_{p}\right) \psi\left(\phi^{-1}\left(\frac{t}{\varepsilon}, \omega\right)\right) \mathrm{d} t .
\end{aligned}
$$

Using the random variable

$$
Z_{\varepsilon}\left(t_{p}, x, \omega\right)=\frac{1}{\sqrt{\varepsilon}} \int_{t_{p}}^{x} \psi\left(\phi^{-1}\left(\frac{t}{\varepsilon}, \omega\right)\right) \mathrm{d} t
$$

we write

$$
\begin{aligned}
\bar{Z}_{\varepsilon}(\alpha, \beta, \omega)-\widetilde{Z}_{\varepsilon}(\alpha, \beta, \omega) & =\sum_{p=1}^{N} \int_{t_{p}}^{t_{p+1}}\left(\mathcal{A}(t)-\mathcal{A}_{p}\right) \frac{d Z_{\varepsilon}\left(t_{p}, t, \omega\right)}{\mathrm{d} t} \mathrm{~d} t \\
& =\sum_{p=1}^{N}\left[\left(\mathcal{A}(t)-\mathcal{A}_{p}\right) Z_{\varepsilon}\left(t_{p}, t, \omega\right)\right]_{t_{p}^{+}}^{t_{p+1}^{-}}-\sum_{p=1}^{N} \int_{t_{p}}^{t_{p+1}} \mathcal{A}^{\prime}(t) Z_{\varepsilon}\left(t_{p}, t, \omega\right) \mathrm{d} t \\
& =-\sum_{p=1}^{N} \int_{t_{p}}^{t_{p+1}} \mathcal{A}^{\prime}(t) Z_{\varepsilon}\left(t_{p}, t, \omega\right) \mathrm{d} t
\end{aligned}
$$

where we have used (5.23). We thus have, using Lemma 5.1, that

$$
\begin{aligned}
\mathbb{E}\left|\bar{Z}_{\varepsilon}(\alpha, \beta, \cdot)-\widetilde{Z}_{\varepsilon}(\alpha, \beta, \cdot)\right| & \leq \sum_{p=1}^{N} \int_{t_{p}}^{t_{p+1}}\left|\mathcal{A}^{\prime}(t)\right| \mathbb{E}\left|Z_{\varepsilon}\left(t_{p}, t, \cdot\right)\right| \mathrm{d} t \\
& \leq C \sum_{p=1}^{N} \int_{t_{p}}^{t_{p+1}}\left|\mathcal{A}^{\prime}(t)\right|\left(\left(t-t_{p}\right)^{2}+\sqrt{\varepsilon}\right)^{1 / 4} \mathrm{~d} t \\
& \leq C \sum_{p=1}^{N}\left(\left(t_{p+1}-t_{p}\right)^{2}+\sqrt{\varepsilon}\right)^{1 / 4} \int_{t_{p}}^{t_{p+1}}\left|\mathcal{A}^{\prime}(t)\right| \mathrm{d} t
\end{aligned}
$$

where $C$ is a constant independent of $\varepsilon$ and $\left(t_{p}\right)_{1 \leq p \leq N+1}$. In view of (5.22), we have

$$
\mathbb{E}\left|\bar{Z}_{\varepsilon}(\alpha, \beta, \cdot)-\widetilde{Z}_{\varepsilon}(\alpha, \beta, \cdot)\right| \leq C\left(\eta^{2}+\sqrt{\varepsilon}\right)^{1 / 4}\left|\mathcal{A}^{\prime}\right|_{L^{1}(\alpha, \beta)} .
$$

Inserting (5.26) in (5.25), we deduce that, for any $\varepsilon$ and $\eta$,

$$
\left|\mathbb{E}\left(\mathrm{e}^{i \xi \bar{Z}_{\varepsilon}(\alpha, \beta, \cdot)}-\mathrm{e}^{i \xi \widetilde{Z}_{\varepsilon}(\alpha, \beta, \cdot)}\right)\right| \leq C|\xi|\left(\eta^{2}+\sqrt{\varepsilon}\right)^{1 / 4}\left|\mathcal{A}^{\prime}\right|_{L^{1}(\alpha, \beta)} .
$$

We next turn to the second term of the right-hand side of (5.24). We recall that $\widetilde{Z}_{\varepsilon}(\alpha, \beta, \omega)$ and $\widetilde{\sigma}(\alpha, \beta)$ depend on $\eta$, through the choice of the function $\widetilde{\mathcal{A}}$. For the parameter $\eta$ that we have chosen, $\widetilde{Z}_{\varepsilon}(\alpha, \beta, \omega)$ converges in law to $\widetilde{Z}_{0}(\alpha, \beta, \omega)$ when $\varepsilon \rightarrow 0$. Thus, there exists $\varepsilon_{0}(\eta)$, that depends on $\eta$ and can be chosen such that $\varepsilon_{0}(\eta) \leq \eta^{4}$, such that, for all $\varepsilon<\varepsilon_{0}(\eta)$,

$$
\left|\mathbb{E}\left(\mathrm{e}^{i \xi \widetilde{Z}_{\varepsilon}(\alpha, \beta, \cdot)}\right)-\mathbb{E}\left(\mathrm{e}^{i \xi \widetilde{Z}_{0}(\alpha, \beta, \cdot)}\right)\right| \leq \eta .
$$


We finally turn to the third term of the right-hand side of $(5.24)$. Since $\bar{Z}_{0}(\alpha, \beta, \omega)$ and $\widetilde{Z}_{0}(\alpha, \beta, \omega)$ are Gaussian random variables, we see that

$$
\mathbb{E}\left(\mathrm{e}^{i \xi \widetilde{Z}_{0}(\alpha, \beta, \cdot)}-\mathrm{e}^{i \xi \bar{Z}_{0}(\alpha, \beta, \cdot)}\right)=\exp \left(-\xi^{2} \widetilde{\sigma}(\alpha, \beta) / 2\right)-\exp \left(-\xi^{2} \bar{\sigma}(\alpha, \beta) / 2\right)
$$

Denoting by $L$ the Lipschitz constant of the function $\sigma \mapsto \exp \left(-\xi^{2} \sigma / 2\right)$ on $[0, \infty)$, we thus have

$$
\left|\mathbb{E}\left(\mathrm{e}^{i \xi \widetilde{Z}_{0}(\alpha, \beta, \cdot)}-\mathrm{e}^{i \xi \bar{Z}_{0}(\alpha, \beta, \cdot)}\right)\right| \leq L|\widetilde{\sigma}(\alpha, \beta)-\bar{\sigma}(\alpha, \beta)|=L \frac{\mathbb{V a r}\left(Y_{0}\right)}{\mathbb{E}\left(\int_{0}^{1} \phi^{\prime}\right)}\left|\|\widetilde{\mathcal{A}}\|_{L^{2}(\alpha, \beta)}^{2}-\|\mathcal{A}\|_{L^{2}(\alpha, \beta)}^{2}\right|
$$

We next write

$$
\|\widetilde{\mathcal{A}}\|_{L^{2}(\alpha, \beta)}^{2}-\|\mathcal{A}\|_{L^{2}(\alpha, \beta)}^{2}=\sum_{p=1}^{N} \int_{t_{p}}^{t_{p+1}}\left(\mathcal{A}_{p}^{2}-\mathcal{A}(t)^{2}\right) \mathrm{d} t=\sum_{p=1}^{N} \int_{t_{p}}^{t_{p+1}}\left(\mathcal{A}_{p}+\mathcal{A}(t)\right)\left(\mathcal{A}_{p}-\mathcal{A}(t)\right) \mathrm{d} t .
$$

In view of (5.23), we have

$$
\forall t \in\left[t_{p}, t_{p+1}\right], \quad \mathcal{A}(t)=\mathcal{A}\left(t_{p+1}^{-}\right)-\int_{t}^{t_{p+1}} \mathcal{A}^{\prime}(s) \mathrm{d} s=\mathcal{A}_{p}-\int_{t}^{t_{p+1}} \mathcal{A}^{\prime}(s) \mathrm{d} s .
$$

Inserting this relation in (5.30), we obtain

$$
\|\widetilde{\mathcal{A}}\|_{L^{2}(\alpha, \beta)}^{2}-\|\mathcal{A}\|_{L^{2}(\alpha, \beta)}^{2}=\sum_{p=1}^{N} \int_{t_{p}}^{t_{p+1}}\left(\mathcal{A}_{p}+\mathcal{A}(t)\right) \int_{t}^{t_{p+1}} \mathcal{A}^{\prime}(s) \mathrm{d} s \mathrm{~d} t .
$$

Thus, in view of the choice (5.22), we have

$$
\begin{aligned}
\|\| \widetilde{\mathcal{A}}\left\|_{L^{2}(\alpha, \beta)}^{2}-\right\| \mathcal{A} \|_{L^{2}(\alpha, \beta)}^{2} \mid & \leq 2\|\mathcal{A}\|_{L^{\infty}(\alpha, \beta)} \sum_{p=1}^{N} \int_{t_{p}}^{t_{p+1}} \int_{t}^{t_{p+1}}\left|\mathcal{A}^{\prime}(s)\right| \mathrm{d} s \mathrm{~d} t \\
& \leq 2\|\mathcal{A}\|_{L^{\infty}(\alpha, \beta)} \sum_{p=1}^{N} \int_{t_{p}}^{t_{p+1}}\left|\mathcal{A}^{\prime}(s)\right|\left(s-t_{p}\right) \mathrm{d} s \\
& \leq 2 \eta\|\mathcal{A}\|_{L^{\infty}(\alpha, \beta)} \sum_{p=1}^{N} \int_{t_{p}}^{t_{p+1}}\left|\mathcal{A}^{\prime}(s)\right| \mathrm{d} s \\
& \leq 2 \eta\|\mathcal{A}\|_{L^{\infty}(\alpha, \beta)}\left|\mathcal{A}^{\prime}\right|_{L^{1}(\alpha, \beta)} .
\end{aligned}
$$

Inserting (5.31) in (5.29), we deduce that

$$
\left|\mathbb{E}\left(\mathrm{e}^{i \xi \widetilde{Z}_{0}(\alpha, \beta, \cdot)}-\mathrm{e}^{i \xi \bar{Z}_{0}(\alpha, \beta, \cdot)}\right)\right| \leq 2 \eta L \frac{\operatorname{Var}\left(Y_{0}\right)}{\mathbb{E}\left(\int_{0}^{1} \phi^{\prime}\right)}\|\mathcal{A}\|_{L^{\infty}(\alpha, \beta)}\left|\mathcal{A}^{\prime}\right|_{L^{1}(\alpha, \beta)} .
$$

Collecting (5.24), (5.27), (5.28) and (5.32), we have, for any $\eta$ and any $\varepsilon<\varepsilon_{0}(\eta) \leq \eta^{4}$, that

$$
\left|\mathbb{E}\left(\mathrm{e}^{i \xi \bar{Z}_{\varepsilon}(\alpha, \beta, \cdot)}\right)-\mathbb{E}\left(\mathrm{e}^{i \xi \bar{Z}_{0}(\alpha, \beta, \cdot)}\right)\right| \leq C|\xi|\left(2 \eta^{2}\right)^{1 / 4}\left|\mathcal{A}^{\prime}\right|_{L^{1}(\alpha, \beta)}+\eta+2 \eta L \frac{\operatorname{Var}\left(Y_{0}\right)}{\mathbb{E}\left(\int_{0}^{1} \phi^{\prime}\right)}\|\mathcal{A}\|_{L^{\infty}(\alpha, \beta)}\left|\mathcal{A}^{\prime}\right|_{L^{1}(\alpha, \beta)} .
$$

The above bound holds for any $\varepsilon<\varepsilon_{0}(\eta)$, and $\eta$ is arbitrary small. In addition, $\left|\mathcal{A}^{\prime}\right|_{L^{1}(\alpha, \beta)}$ is independent from $\eta$, even though the set of points $\left(t_{p}\right)_{1 \leq p \leq N+1}$ depends on $\eta$. This means that

$$
\lim _{\varepsilon \rightarrow 0}\left|\mathbb{E}\left(e^{i \xi \bar{Z}_{\varepsilon}(\alpha, \beta, \omega)}\right)-\mathbb{E}\left(e^{i \xi \bar{Z}_{0}(\alpha, \beta, \omega)}\right)\right|=0,
$$

hence $\bar{Z}_{\varepsilon}(\alpha, \beta, \omega)$ converges in law to $\bar{Z}_{0}(\alpha, \beta, \omega)$. This concludes the proof of Lemma 4.6. 


\subsection{Proof of Lemma 4.7}

According to the definition (4.37), we have:

$$
\left|H_{\varepsilon}(x, \omega)-H_{\varepsilon}(y, \omega)\right| \leq\left|\frac{1}{\sqrt{\varepsilon}} \int_{y}^{x} \mathcal{A}_{1}(t) \psi\left(\phi^{-1}\left(\frac{t}{\varepsilon}, \omega\right)\right) \mathrm{d} t\right|+|x-y|\left|\frac{1}{\sqrt{\varepsilon}} \int_{0}^{1} \mathcal{A}_{2}(t) \psi\left(\phi^{-1}\left(\frac{t}{\varepsilon}, \omega\right)\right) \mathrm{d} t\right| .
$$

Thus, for any $p \in \mathbb{N}^{\star}$, using Lemma 4.1 and the fact that $|y-x| \leq 1$, we have

$$
\begin{aligned}
\mathbb{E}\left[\left|H_{\varepsilon}(x, \cdot)-H_{\varepsilon}(y, \cdot)\right|^{2 p}\right] \leq & C_{p} \mathbb{E}\left[\left|\frac{1}{\sqrt{\varepsilon}} \int_{y}^{x} \mathcal{A}_{1}(t) \psi\left(\phi^{-1}\left(\frac{t}{\varepsilon}, \cdot\right)\right) \mathrm{d} t\right|^{2 p}\right] \\
& +C_{p}|x-y|^{2 p} \mathbb{E}\left[\left|\frac{1}{\sqrt{\varepsilon}} \int_{0}^{1} \mathcal{A}_{2}(t) \psi\left(\phi^{-1}\left(\frac{t}{\varepsilon}, \cdot\right)\right) \mathrm{d} t\right|^{2 p}\right] \\
\leq & C_{p}\left(|x-y|^{p}+\varepsilon^{(p-1) / 2}\right)+C_{p}|x-y|^{2 p} \\
\leq & C_{p}\left(|x-y|^{p}+\varepsilon^{(p-1) / 2}\right) .
\end{aligned}
$$

This concludes the proof of (4.38).

Assume now that $|x-y| \leq \varepsilon$. We infer from (5.33) that

$$
\left|H_{\varepsilon}(x, \omega)-H_{\varepsilon}(y, \omega)\right| \leq\left|\frac{x-y}{\sqrt{\varepsilon}}\right|\|\psi\|_{L^{\infty}(\mathbb{R})}\left(\left\|\mathcal{A}_{1}\right\|_{L^{\infty}(0,1)}+\left\|\mathcal{A}_{2}\right\|_{L^{\infty}(0,1)}\right) \leq C \sqrt{|x-y|},
$$

where $C$ is a deterministic constant independent of $\varepsilon, x$ and $y$. This concludes the proof of (4.39), and hence the proof of Lemma 4.7 .

\section{Approximation of the homogenized matrix}

The aim of this section is to prove our second main result, Theorem 3.4 of Section 3.2. Since the approach described in Section 3.2 mimicks the approach proposed in [10], our proof essentially follows the arguments used in [10]. Because our proof is involved, we feel that it is useful to first recall the arguments of [10] in Section 6.1. We then collect some technical results in Section 6.2, before turning to the actual proof of Theorem 3.4 in Sections 6.3 and 6.4.

\subsection{Convergence proof in the classical random homogenization setting}

Consider the classical random homogenization problem

$$
-\operatorname{div}\left[A\left(\frac{x}{\varepsilon}, \omega\right) \nabla u^{\varepsilon}(x, \omega)\right]=f(x) \text { in } \mathcal{D}, \quad u^{\varepsilon}(\cdot, \omega)=0 \text { on } \partial \mathcal{D},
$$

where $\mathcal{D}$ is a bounded open set of $\mathbb{R}^{d}, f \in L^{2}(\mathcal{D})$, and $A$ is a stationary matrix in the sense of (2.1), satisfying classical coercivity and boundedness properties. The associated homogenized problem is (2.7), where the homogenized matrix is given by

$$
\forall 1 \leq i, j \leq d, \quad A_{i j}^{\star}=\mathbb{E}\left[\int_{Q} e_{i}^{T} A(y, \cdot)\left(e_{j}+\nabla w_{e_{j}}(y, \cdot)\right) \mathrm{d} y\right],
$$

where $Q=(0,1)^{d}$ and where, for all $p \in \mathbb{R}^{d}, w_{p}$ solves the corrector problem

$$
\left\{\begin{array}{l}
-\operatorname{div}\left[A(y, \omega)\left(p+\nabla w_{p}(y, \omega)\right)\right]=0 \text { in } \mathbb{R}^{d}, \\
\nabla w_{p} \text { is stationary in the sense of }(2.1), \quad \mathbb{E}\left(\int_{Q} \nabla w_{p}(y, \cdot) \mathrm{d} y\right)=0 .
\end{array}\right.
$$


In [10], the following approximation strategy is proposed: introduce the approximate corrector $w_{p}^{N}(\cdot, \omega)$ as the $Q_{N}$-periodic function satisfying:

$$
\text { for all } \psi Q_{N} \text {-periodic, } \quad \int_{Q_{N}}(\nabla \psi)^{T} A(\cdot, \omega)\left(p+\nabla w_{p}^{N}(\cdot, \omega)\right)=0 \quad \text { with } \int_{Q_{N}} w_{p}^{N}(\cdot, \omega)=0
$$

and the approximate homogenized matrix $A_{N}^{\star}(\omega)$ defined by, for any $1 \leq i, j \leq d$,

$$
\left[A_{N}^{\star}(\omega)\right]_{i j}=\frac{1}{\left|Q_{N}\right|} \int_{Q_{N}} e_{i}^{T} A(\cdot, \omega)\left(e_{j}+\nabla w_{e_{j}}^{N}(\cdot, \omega)\right) .
$$

Then, see Theorem 1 of [10], we have that

$$
\lim _{N \rightarrow \infty} A_{N}^{\star}(\omega)=A^{\star} \quad \text { almost surely. }
$$

A key ingredient for the proof of (6.3) is the following classical homogenization result, see Theorem 5.2 page 151 of [15]:

Theorem 6.1. Let $A^{\varepsilon}$ be a sequence of matrices that $G$-converges to $A^{\star}$ in a domain $V$, and let $V_{1}$ be an arbitrary subdomain of $V$. Let $p \in \mathbb{R}^{d}$, and assume that the functions $w_{p}^{\varepsilon} \in H^{1}\left(V_{1}\right)$ satisfy the conditions

$$
w_{p}^{\varepsilon} \rightarrow w_{p}^{\infty} \quad \text { weakly in } H^{1}\left(V_{1}\right), \quad \text { and } \quad-\operatorname{div}\left[A^{\varepsilon}\left(p+\nabla w_{p}^{\varepsilon}\right)\right]=0 \quad \text { in } \mathcal{D}^{\prime}\left(V_{1}\right) .
$$

Then we have that

$$
A^{\varepsilon}\left(p+\nabla w_{p}^{\varepsilon}\right) \rightarrow A^{\star}\left(p+\nabla w_{p}^{\infty}\right) \text { weakly in }\left(L^{2}\left(V_{1}\right)\right)^{d},
$$

where $w_{p}^{\infty}$ satisfies

$$
-\operatorname{div}\left[A^{\star}\left(p+\nabla w_{p}^{\infty}\right)\right]=0 \quad \text { in } \mathcal{D}^{\prime}\left(V_{1}\right) .
$$

The proof of (6.3) goes as follows (see [10] for details). The rescaled corrector

$$
w_{0, p}^{N}(x, \omega):=\frac{1}{N} w_{p}^{N}(N x, \omega)
$$

is shown to satisfy the a priori bound $\left\|w_{0, p}^{N}(\cdot, \omega)\right\|_{H^{1}(Q)} \leq C$, where $C$ is a deterministic constant independent from $N$. We thus deduce that, almost surely, there exists a $Q$-periodic function $w_{0, p}^{\infty}(\cdot, \omega) \in H^{1}(Q)$ such that (up to the extraction of a subsequence)

$$
w_{0, p}^{N}(\cdot, \omega) \rightarrow w_{0, p}^{\infty}(\cdot, \omega) \quad \text { weakly in } H^{1}(Q) .
$$

Consider a $Q$-periodic function $\psi \in H^{1}(Q)$. Choosing $\psi_{N}(y)=\psi(y / N)$ as test function in (6.1), we obtain

$$
\int_{Q}(\nabla \psi)^{T} A(N \cdot, \omega)\left(p+\nabla w_{0, p}^{N}(\cdot, \omega)\right)=0 .
$$

We are then in position to use Theorem 6.1 on the domain $V_{1}=Q$. We thus get that $A(N \cdot, \omega)\left(p+\nabla w_{0, p}^{N}\right)$ weakly converges to $A^{\star}\left(p+\nabla w_{0, p}^{\infty}\right)$ in $\left(L^{2}(Q)\right)^{d}$. We then infer from (6.4) that, for any $Q$-periodic function $\psi$, we have

$$
\int_{Q}(\nabla \psi)^{T} A^{\star}\left(p+\nabla w_{0, p}^{\infty}(\cdot, \omega)\right)=0 .
$$

This implies that $\nabla w_{0, p}^{\infty}(\cdot, \omega)=0$ a.s. The limit being unique, all the sequence $\nabla w_{0, p}^{N}(\cdot, \omega)$ weakly converges to $\nabla w_{0, p}^{\infty}(\cdot, \omega)$. Using the same weak $L^{2}$ convergence as above, we deduce from (6.2) that

$$
\left[A_{N}^{\star}(\omega)\right]_{i j}=\int_{Q} e_{i}^{T} A(N \cdot, \omega)\left(e_{j}+\nabla w_{0, e_{j}}^{N}(\cdot, \omega)\right) \rightarrow \int_{Q} e_{i}^{T} A^{\star}\left(e_{j}+\nabla w_{0, e_{j}}^{\infty}(\cdot, \omega)\right)=\left[A^{\star}\right]_{i j} .
$$

This concludes the proof of (6.3). 


\subsection{Some technical ingredients for our analysis}

A key ingredient to prove Theorem 3.4 is to find an appropriate domain on which to apply Theorem 6.1 . The following lemmas are useful for that purpose.

We first recall, see Lemme 2.1 of $[7]$, that $\frac{1}{N} \phi(N \cdot, \omega)$ converges to $\mathbb{E}\left(\int_{Q} \nabla \phi\right) \cdot$ in $L_{\text {loc }}^{\infty}\left(\mathbb{R}^{d}\right)$ almost surely. Likewise, in view of the proof of Lemme 2.2 of [7], we have that $\frac{1}{N} \phi^{-1}(N \cdot, \omega)$ converges to $\left[\mathbb{E}\left(\int_{Q} \nabla \phi\right)\right]^{-1}$. in $L_{\text {loc }}^{\infty}\left(\mathbb{R}^{d}\right)$ almost surely. The functions being smooth, we thus have that, for any compact $K$,

$$
\lim _{N \rightarrow \infty}\left\|\frac{1}{N} \phi(N \cdot, \omega)-\mathbb{E}\left(\int_{Q} \nabla \phi\right) \cdot\right\|_{C^{0}(K)}=\lim _{N \rightarrow \infty}\left\|\frac{1}{N} \phi^{-1}(N \cdot, \omega)-\left[\mathbb{E}\left(\int_{Q} \nabla \phi\right)\right]^{-1} \cdot\right\|_{C^{0}(K)}=0 \text { a.s. }
$$

As pointed out in the proof of Lemme 2.2 of [7], a consequence of the above fact is that

$$
\lim _{N \rightarrow \infty}\left\|\mathbf{1}_{\frac{1}{N} \phi(N Q, \omega)}-\mathbf{1}_{\mathbb{E}\left(\int_{Q} \nabla \phi\right) Q}\right\|_{L^{1}\left(\mathbb{R}^{d}\right)}=0 \text { a.s. }
$$

This can be shown by first assuming that $\phi(0, \omega)=0$, and using a regularization of the indicator functions. The general case $\phi(0, \omega) \neq 0$ next follows as an easy consequence.

The first ingredient we need to prove Theorem 3.4 is the following lemma, which is somewhat related with the above results:

Lemma 6.2. Let $\phi$ be a diffeomorphism that satisfies (2.4), (2.5) and (2.6). For any compact set $K$ that is a proper subset of the open set $\mathbb{E}\left(\int_{Q} \nabla \phi\right) Q$, and for almost all $\omega$, there exists $N_{0}(\omega) \in \mathbb{N}$ such that

$$
\forall N \geq N_{0}(\omega), \quad \stackrel{\circ}{K} \subset \frac{1}{N} \phi\left(Q_{N}, \omega\right),
$$

where $\stackrel{\circ}{K}$ denotes the interior of the set $K$ and, we recall, $Q_{N}=N Q$.

The following easy result is useful to prove Lemma 6.2:

Lemma 6.3. Let $\phi$ be a diffeomorphism that satisfies (2.4) and (2.5). Then there exists a deterministic constant $L_{\mathrm{Lip}}$ such that the diffeomorphism $\phi^{-1}(\cdot, \omega)$ is Lipschitz with that constant.

Proof of Lemma 6.3. We infer from (2.5) that $\nabla \phi^{T} \nabla \phi$, which is a symmetric matrix and therefore diagonalizable, has a bounded spectrum. The assumption (2.4) then implies that the eigenvalues of $\nabla \phi^{T} \nabla \phi$ are bounded away from 0 . Hence, there exists a deterministic constant $c>0$ such that for all $\xi \in \mathbb{R}^{d}$ we have

$$
\xi^{T}(\nabla \phi(x, \omega))^{T} \nabla \phi(x, \omega) \xi \geq c|\xi|^{2} \quad \text { a.s., a.e. on } \mathbb{R}^{d} .
$$

For any $\bar{\xi} \in \mathbb{R}^{d}$, we set $\xi=(\nabla \phi(x, \omega))^{-1} \bar{\xi}$ and obtain that

$$
\left|(\nabla \phi(x, \omega))^{-1} \bar{\xi}\right| \leq c^{-1 / 2}|\bar{\xi}|
$$

The diffeomorphism $\phi^{-1}(\cdot, \omega)$ is thus Lipshitz with the deterministic constant $c^{-1 / 2}$.

Proof of Lemma 6.2. Let $K$ be a proper subset of the open set $\mathbb{E}\left(\int_{Q} \nabla \phi\right) Q$, and let us fix $\omega$ such that

$$
\frac{1}{N} \phi^{-1}(N \cdot, \omega) \text { converges to }\left[\mathbb{E}\left(\int_{Q} \nabla \phi\right)\right]^{-1} \cdot \text { in } C^{0}(K) .
$$

In view of (6.6), we know that (6.9) holds for almost all $\omega$. 
We prove Lemma 6.2 by contradiction. Suppose that, for all $N_{0} \in \mathbb{N}$, there exists $N\left(N_{0}, \omega\right) \geq N_{0}$ such that $\stackrel{\circ}{K}$ is not included in $\frac{1}{N\left(N_{0}, \omega\right)} \phi\left(Q_{N\left(N_{0}, \omega\right)}, \omega\right)$. Otherwise stated, there exist $N\left(N_{0}, \omega\right)$ and $z\left(N_{0}, \omega\right)$ such that

$$
z\left(N_{0}, \omega\right) \in \stackrel{\circ}{K} \quad \text { and } \quad z\left(N_{0}, \omega\right) \notin \frac{1}{N\left(N_{0}, \omega\right)} \phi\left(N\left(N_{0}, \omega\right) Q, \omega\right) .
$$

Introduce $y\left(N_{0}, \omega\right)=\frac{1}{N\left(N_{0}, \omega\right)} \phi^{-1}\left(N\left(N_{0}, \omega\right) z\left(N_{0}, \omega\right), \omega\right)$. We thus have that

$$
y\left(N_{0}, \omega\right) \notin Q .
$$

We now pass to the limit $N_{0} \rightarrow \infty$. Observing that $z\left(N_{0}, \omega\right)$ belongs to the compact set $K$, we deduce that $\left\{z\left(N_{0}, \omega\right)\right\}_{N_{0} \in \mathbb{N}}$ is a bounded sequence and thus converges, up to the extraction of a subsequence, toward some $\bar{z}(\omega) \in K$.

Let us now show that $\left\{y\left(N_{0}, \omega\right)\right\}_{N_{0} \in \mathbb{N}}$ is also a bounded sequence. Using the fact that the diffeomorphism $\phi^{-1}(\cdot, \omega)$ is a Lipschitz mapping with a deterministic constant $L_{\text {Lip }}$ (see Lem. 6.3), we write

$$
\left|y\left(N_{0}, \omega\right)\right|=\frac{1}{N\left(N_{0}, \omega\right)}\left|\phi^{-1}\left(N\left(N_{0}, \omega\right) z\left(N_{0}, \omega\right), \omega\right)\right| \leq L_{\text {Lip }}\left|z\left(N_{0}, \omega\right)\right|+\frac{1}{N\left(N_{0}, \omega\right)}\left|\phi^{-1}(0, \omega)\right| .
$$

We deduce that, almost surely, $\left\{y\left(N_{0}, \omega\right)\right\}_{N_{0} \in \mathbb{N}}$ is a bounded sequence and thus converges, up to the extraction of a subsequence, toward some $\bar{y}(\omega)$. In view of (6.10), and since $Q$ is an open set, we have that $\bar{y}(\omega) \notin Q$.

We now claim that

$$
\bar{z}(\omega)=\mathbb{E}\left(\int_{Q} \nabla \phi\right) \bar{y}(\omega)
$$

Indeed, we write that

$$
\begin{aligned}
& \left|y\left(N_{0}, \omega\right)-\left[\mathbb{E}\left(\int_{Q} \nabla \phi\right)\right]^{-1} \bar{z}(\omega)\right| \\
\leq & \left|\frac{1}{N\left(N_{0}, \omega\right)} \phi^{-1}\left(N\left(N_{0}, \omega\right) z\left(N_{0}, \omega\right), \omega\right)-\left[\mathbb{E}\left(\int_{Q} \nabla \phi\right)\right]^{-1} z\left(N_{0}, \omega\right)\right| \\
& +\left|\left[\mathbb{E}\left(\int_{Q} \nabla \phi\right)\right]^{-1} z\left(N_{0}, \omega\right)-\left[\mathbb{E}\left(\int_{Q} \nabla \phi\right)\right]^{-1} \bar{z}(\omega)\right| \\
\leq & \| \frac{1}{N\left(N_{0}, \omega\right)} \phi^{-1}\left(N\left(N_{0}, \omega\right) \cdot \omega\right)-\left[\mathbb{E}\left(\int_{Q} \nabla \phi\right)\right]^{-1} \cdot||_{C^{0}(K)}+C\left|z\left(N_{0}, \omega\right)-\bar{z}(\omega)\right| .
\end{aligned}
$$

Both terms converge to 0 when $N_{0} \rightarrow \infty$, respectively in view of (6.9) and of the definition of $\bar{z}(\omega)$. By definition of $\bar{y}(\omega)$, we deduce $(6.11)$.

We now reach a contradiction since $\bar{z}(\omega) \in K \subset \mathbb{E}\left(\int_{Q} \nabla \phi\right) Q$ whereas $\bar{y}(\omega) \notin Q$. This concludes the proof of Lemma 6.2. 
The second ingredient we need to prove Theorem 3.4 is the following lemma:

Lemma 6.4. Let $\phi$ be a diffeomorphism that satisfies (2.4) and (2.5). There exists an open set $\widetilde{Q}(\omega)$ and some $k(\omega) \in \mathbb{N}$ such that

$$
\begin{aligned}
\forall N \in \mathbb{N}^{\star}, & \frac{1}{N} \phi\left(Q_{N}, \omega\right) \subset \widetilde{Q}(\omega), \\
& \mathbb{E}\left(\int_{Q} \nabla \phi\right) Q \subset \widetilde{Q}(\omega), \\
\forall N \in \mathbb{N}^{\star}, \quad & \widetilde{Q}(\omega) \subset \frac{1}{N} \phi\left(Q_{k(\omega) N}, \omega\right) .
\end{aligned}
$$

Proof. The first assertion relies on the fact that, in view of (2.5), we have

$$
\forall N \in \mathbb{N}^{\star}, \quad \frac{1}{N}|\phi(N x, \omega)| \leq M|x|+\frac{1}{N}\left|\phi^{-1}(0, \omega)\right| \leq M+\left|\phi^{-1}(0, \omega)\right| \quad \text { a.s., a.e. on } Q \text {. }
$$

It is thus sufficient to choose $\widetilde{Q}(\omega)$ such that $\left[-M-\left|\phi^{-1}(0, \omega)\right|, M+\left|\phi^{-1}(0, \omega)\right|\right]^{d} \subset \widetilde{Q}(\omega)$. Upon choosing a larger $\widetilde{Q}(\omega)$, the second assertion is also satisfied. Now that $\widetilde{Q}(\omega)$ is chosen, we show that we can choose $k(\omega)$ such that the third assertion is satisfied. Using Lemma 6.3, we see that, almost surely,

$$
\forall N \in \mathbb{N}^{\star}, \quad \frac{1}{N}\left|\phi^{-1}(N x, \omega)\right| \leq L_{\text {Lip }}|x|+\frac{1}{N}|\phi(0, \omega)| \leq L_{\text {Lip }}|x|+|\phi(0, \omega)| \quad \text { a.e. on } \mathbb{R}^{d} .
$$

There thus exists $k(\omega)$ such that, for any $N \in \mathbb{N}^{\star}$, we have $\frac{1}{N} \phi^{-1}(N \widetilde{Q}(\omega), \omega) \subset Q_{k(\omega)}$. This implies the third assertion and concludes the proof.

\subsection{Proof of Theorem 3.4}

To simplify the notation, we introduce the matrix

$$
\alpha:=\mathbb{E}\left(\int_{Q} \nabla \phi\right) \in \mathbb{R}^{d \times d} .
$$

As pointed out in Remark 1.9 of [8], we have that

$$
\operatorname{det} \alpha=\mathbb{E}\left(\int_{Q} \operatorname{det} \nabla \phi\right) .
$$

We hence deduce from (2.4) that

$$
\operatorname{det} \alpha \geq \nu>0 .
$$

We also introduce the matrix $\beta \in \mathbb{R}^{d \times d}$ defined by

$$
\beta=\mathbb{E}\left[\int_{\phi(Q, \cdot)}\left(\nabla \phi\left(\phi^{-1}(x, \cdot), \cdot\right)\right)^{-1} \mathrm{~d} x\right]=\mathbb{E}\left[\int_{Q} \operatorname{det}(\nabla \phi)(\nabla \phi)^{-1}\right] .
$$

The proof of the following lemma, useful for proving Theorem 3.4, is postponed until Section 6.4.

Lemma 6.5. The constant matrix $\beta A^{\star} \alpha^{-T}$ is coercive.

The proof of Theorem 3.4 is composed of four steps. In Step 1, we introduce a rescaled corrector, denoted $w_{0, p}^{N}(\cdot, \omega)$ (see $(6.19)$ below), and show that it converges toward some function $w_{0, p}^{\infty}(\cdot, \omega)$ weakly in $H^{1}$. Then, in Step 2, we prove that $w_{0, p}^{\infty}(\cdot, \omega)$ is $\alpha Q$-periodic. Next, in Step 3, we show that $w_{0, p}^{\infty}(\cdot, \omega)$ solves the equation 
$-\operatorname{div}\left[B \nabla w_{0, p}^{\infty}\right]=0$ in $\mathbb{R}^{d}$ for a constant deterministic matrix $B$ (see (6.34) below for a precise statement). Combining these results and using Lemma 6.5 , we conclude that $\nabla w_{0, p}^{\infty} \equiv 0$. This is a key ingredient to prove, in Step 4, that the random approximation $A_{N}^{\star}(\omega)$ indeed converges to the homogenized matrix $A^{\star}$ almost surely.

Step 1. Introduction of a rescaled corrector $w_{0, p}^{N}$, and convergence of $w_{0, p}^{N}$ to some $w_{0, p}^{\infty}$.

We first establish some a priori bounds. Taking $\widetilde{\psi}=\widetilde{w}_{p}^{N}$ as test function in (3.9), and using (2.4) and (2.5), we see that

$$
\left\|(\nabla \phi(\cdot, \omega))^{-T} \nabla \widetilde{w}_{p}^{N}(\cdot, \omega)\right\|_{L^{2}\left(Q_{N}\right)} \leq C \sqrt{\left|Q_{N}\right|},
$$

where $C$ is a deterministic constant independent from $N$. Using again (2.5), we deduce that

$$
\left\|\nabla \widetilde{w}_{p}^{N}(\cdot, \omega)\right\|_{L^{2}\left(Q_{N}\right)} \leq C \sqrt{\left|Q_{N}\right|} .
$$

Let $k \in \mathbb{N}$. Since $\widetilde{w}_{p}^{N}$ is $Q_{N}$-periodic, we infer from the above bound that

$$
\left\|\nabla \widetilde{w}_{p}^{N}(\cdot, \omega)\right\|_{L^{2}\left(Q_{k N}\right)} \leq C \sqrt{\left|Q_{k N}\right|},
$$

where $C$ is a deterministic constant independent from $N$ and $k$.

We now introduce the following rescaled corrector:

$$
w_{0, p}^{N}(x, \omega)=\frac{1}{N} w_{p}^{N}(N x, \omega),
$$

where, we recall $w_{p}^{N}(y, \omega)=\widetilde{w}_{p}^{N}\left(\phi^{-1}(y, \omega), \omega\right)$. Using (2.5) and (6.8), we infer from (6.18) that

$$
\left\|\nabla w_{0, p}^{N}(\cdot, \omega)\right\|_{L^{2}\left(\frac{1}{N} \phi\left(Q_{k N}, \omega\right)\right)} \leq C k^{d / 2}
$$

where $C$ is a deterministic constant independent from $N$ and $k$. We now choose $k$ in the above bound equal to the integer $k(\omega)$ defined in Lemma 6.4. We infer from the above bound and (6.14) that

$$
\forall N \in \mathbb{N}^{\star}, \quad\left\|\nabla w_{0, p}^{N}(\cdot, \omega)\right\|_{L^{2}(\widetilde{Q}(\omega))} \leq C(\omega) .
$$

Recall that the solution $\widetilde{w}_{p}^{N}$ to (3.9) is unique up to an additive constant. We now fix this constant by choosing $\widetilde{w}_{p}^{N}$ such that $\int_{N \widetilde{Q}(\omega)} w_{p}^{N}(\cdot, \omega)=0$, where the set $\widetilde{Q}(\omega)$ is defined in Lemma 6.4. In view of (6.19), this means that $\int_{\widetilde{Q}(\omega)} w_{0, p}^{N}(\cdot, \omega)=0$. Using $(6.20)$ and the Poincaré-Wirtinger inequality, we deduce that there exists $C(\omega)$ such that

$$
\forall N \in \mathbb{N}^{\star}, \quad\left\|w_{0, p}^{N}(\cdot, \omega)\right\|_{H^{1}(\widetilde{Q}(\omega))} \leq C(\omega) .
$$

This implies that, almost surely, there exists $w_{0, p}^{\infty}(\cdot, \omega) \in H^{1}(\widetilde{Q}(\omega))$ such that (up to the extraction of a subsequence)

$$
w_{0, p}^{N}(\cdot, \omega) \rightarrow w_{0, p}^{\infty}(\cdot, \omega) \quad \text { weakly in } H^{1}(\widetilde{Q}(\omega)),
$$

and, using the Rellich Theorem, that

$$
w_{0, p}^{N}(\cdot, \omega) \rightarrow w_{0, p}^{\infty}(\cdot, \omega) \quad \text { strongly in } L^{2}(\widetilde{Q}(\omega)) .
$$


Step 2. $w_{0, p}^{\infty}$ is $\alpha Q$-periodic.

We infer from (6.19) that

$$
w_{0, p}^{N}\left(\frac{\phi(N y, \omega)}{N}, \omega\right)=\frac{1}{N} w_{p}^{N}(\phi(N y, \omega), \omega)=\frac{1}{N} \widetilde{w}_{p}^{N}(N y, \omega)
$$

Since the function $\widetilde{w}_{p}^{N}$ is $Q_{N}$-periodic, we see that the function $y \mapsto w_{0, p}^{N}\left(\frac{\phi(N y, \omega)}{N}, \omega\right)$ is $Q$-periodic. Hence, for any $k \in \mathbb{Z}^{d}$, we have, almost surely,

$$
\begin{aligned}
\int_{Q}\left[w_{0, p}^{\infty}(\alpha y, \omega)-w_{0, p}^{\infty}(\alpha(y+k), \omega)\right]^{2} \mathrm{~d} y & \leq 2 \int_{Q}\left[w_{0, p}^{\infty}(\alpha y, \omega)-w_{0, p}^{N}\left(\frac{\phi(N y, \omega)}{N}, \omega\right)\right]^{2} \mathrm{~d} y \\
& +2 \int_{Q}\left[w_{0, p}^{N}\left(\frac{\phi(N(y+k), \omega)}{N}, \omega\right)-w_{0, p}^{\infty}(\alpha(y+k), \omega)\right]^{2} \mathrm{~d} y .
\end{aligned}
$$

We now show that both terms in the above right-hand side converge to 0 when $N \rightarrow \infty$. It is sufficient to consider the first term. Let us fix $\eta>0$.

We observe that the first term in the above right-hand side satisfies

$$
\int_{Q}\left[w_{0, p}^{\infty}(\alpha y, \omega)-w_{0, p}^{N}\left(\frac{\phi(N y, \omega)}{N}, \omega\right)\right]^{2} \mathrm{~d} y \leq 2\left[C_{0}^{N}(\omega)+C_{1}^{N}(\omega)\right],
$$

where

$$
\begin{aligned}
& C_{0}^{N}(\omega)=\int_{Q}\left[w_{0, p}^{\infty}(\alpha y, \omega)-w_{0, p}^{\infty}\left(\frac{\phi(N y, \omega)}{N}, \omega\right)\right]^{2} \mathrm{~d} y \\
& C_{1}^{N}(\omega)=\int_{Q}\left[w_{0, p}^{\infty}\left(\frac{\phi(N y, \omega)}{N}, \omega\right)-w_{0, p}^{N}\left(\frac{\phi(N y, \omega)}{N}, \omega\right)\right]^{2} \mathrm{~d} y .
\end{aligned}
$$

To show that $C_{0}^{N}(\omega)$ converges to 0 , we use the fact that the function $\frac{\phi(N y, \omega)}{N}$ converges to the function $\alpha y$ in $L_{\text {loc }}^{\infty}\left(\mathbb{R}^{d}\right)$ almost surely, see Lemme 2.1 of [7], and a regularization argument. Since $w_{0, p}^{\infty}(\cdot, \omega) \in H^{1}(\widetilde{Q}(\omega))$, there exists $w_{\eta}^{\infty}(\cdot, \omega) \in C^{\infty}(\widetilde{Q}(\omega))$ such that

$$
\left\|w_{\eta}^{\infty}(\cdot, \omega)-w_{0, p}^{\infty}(\cdot, \omega)\right\|_{L^{2}(\widetilde{Q}(\omega))} \leq \eta
$$

We then write that

$$
C_{0}^{N}(\omega) \leq C_{00}^{N}(\omega)+C_{01}^{N}(\omega)+C_{02}^{N}(\omega)
$$

where

$$
\begin{aligned}
C_{00}^{N}(\omega) & =\int_{Q}\left[w_{0, p}^{\infty}(\alpha y, \omega)-w_{\eta}^{\infty}(\alpha y, \omega)\right]^{2} \mathrm{~d} y \\
C_{01}^{N}(\omega) & =\int_{Q}\left[w_{\eta}^{\infty}(\alpha y, \omega)-w_{\eta}^{\infty}\left(\frac{\phi(N y, \omega)}{N}, \omega\right)\right]^{2} \mathrm{~d} y \\
C_{02}^{N}(\omega) & =\int_{Q}\left[w_{\eta}^{\infty}\left(\frac{\phi(N y, \omega)}{N}, \omega\right)-w_{0, p}^{\infty}\left(\frac{\phi(N y, \omega)}{N}, \omega\right)\right]^{2} \mathrm{~d} y .
\end{aligned}
$$


We infer from (6.25) and (6.13) that

$$
\forall N \in \mathbb{N}^{\star}, \quad C_{00}^{N}(\omega)=\frac{1}{\sqrt{\operatorname{det} \alpha}}\left\|w_{0, p}^{\infty}(\cdot, \omega)-w_{\eta}^{\infty}(\cdot, \omega)\right\|_{L^{2}(\alpha Q)} \leq \frac{1}{\sqrt{\operatorname{det} \alpha}} \eta .
$$

Likewise, we infer from (6.25), (6.12) and (2.4) that

$$
\forall N \in \mathbb{N}^{\star}, \quad C_{02}^{N}(\omega) \leq \frac{1}{\sqrt{\nu}}\left\|w_{\eta}^{\infty}(\cdot, \omega)-w_{0, p}^{\infty}(\cdot, \omega)\right\|_{L^{2}\left(\frac{1}{N} \phi\left(Q_{N}, \omega\right)\right)} \leq \frac{1}{\sqrt{\nu}} \eta .
$$

We now turn to $C_{01}^{N}(\omega)$. Using the fact that $w_{\eta}^{\infty}(\cdot, \omega) \in C^{\infty}(\widetilde{Q}(\omega))$ and that the function $\frac{\phi(N y, \omega)}{N}$ converges to the function $\alpha y$ in $L_{\text {loc }}^{\infty}\left(\mathbb{R}^{d}\right)$ almost surely, we obtain that $C_{01}^{N}(\omega)$ converges to zero as $N$ goes to infinity, almost surely. We thus can choose $N(\eta, \omega) \in \mathbb{N}$ such that

$$
\forall N \geq N(\eta, \omega), \quad C_{01}^{N}(\omega) \leq \eta
$$

Collecting (6.26), (6.27), (6.29) and (6.28), we conclude that

$$
C_{0}^{N}(\omega) \rightarrow 0 \text { as } N \text { goes to infinity, almost surely. }
$$

We next turn to $C_{1}^{N}(\omega)$, which is non-negative by definition, and satisfies, using (6.12) and (6.22),

$$
C_{1}^{N}(\omega)=\int_{\widetilde{Q}(\omega)} \mathbf{1}_{\frac{\phi\left(Q_{N}, \omega\right)}{N}} \frac{1}{\operatorname{det}(\nabla \phi)}\left[w_{0, p}^{\infty}(y, \omega)-w_{0, p}^{N}(y, \omega)\right]^{2} \mathrm{~d} y \leq \frac{1}{\nu}\left\|w_{0, p}^{\infty}(\cdot, \omega)-w_{0, p}^{N}(\cdot, \omega)\right\|_{L^{2}(\widetilde{Q}(\omega))}^{2} \rightarrow 0
$$

as $N \rightarrow \infty$. Collecting (6.23), (6.24), (6.30) and (6.31), we deduce that, almost surely,

$$
\forall k \in \mathbb{Z}^{d}, \quad \int_{Q}\left[w_{0, p}^{\infty}(\alpha y, \omega)-w_{0, p}^{\infty}(\alpha(y+k), \omega)\right]^{2} \mathrm{~d} y=0 .
$$

The function $w_{0, p}^{\infty}(\cdot, \omega)$ is thus $\alpha Q$-periodic (almost surely).

Step 3. $w_{0, p}^{\infty}$ solves $-\operatorname{div}\left[B \nabla w_{0, p}^{\infty}\right]=0$ in $\mathcal{D}^{\prime}\left(\mathbb{R}^{d}\right)$ where $B$ is a constant deterministic matrix.

In the two above steps, we closely followed the proof strategy of [10] recalled in Section 6.1. This Step 3 follows a slightly different pattern, and is more involved than the corresponding argument in [10], which consists in showing the weak formulation (6.5). As pointed out above, the difficulty comes from identifying an appropriate domain, independent of $N$, on which to apply Theorem 6.1. To circumvent this difficulty, we work on the entire space $\mathbb{R}^{d}$, with test functions of compact support.

Introduce a test function $\psi \in \mathcal{D}\left(\mathbb{R}^{d}\right)$, and define the $Q_{N}$-periodic function

$$
\psi_{N}(y):=\sum_{k \in \mathbb{Z}^{d}} \psi\left(\frac{1}{N} y-k\right)
$$

We note that, for any $y \in Q_{N}$, only a finite number of terms in the above sum do not vanish, and that this number of terms only depends on the support of $\psi$ and is thus independent of $N$.

Choosing $\psi_{N}$ as test function in (3.9), we write

$$
\int_{Q_{N}} \operatorname{det}(\nabla \phi(y, \omega))\left(\sum_{k \in \mathbb{Z}^{d}} \nabla \psi\left(\frac{1}{N} y-k\right)\right)^{T}(\nabla \phi(y, \omega))^{-1} A_{\text {per }}(y)\left(p+(\nabla \phi)^{-T}(y, \omega) \nabla \widetilde{w}_{p}^{N}(y, \omega)\right) \mathrm{d} y=0 .
$$


After the change of variable $z=\phi(y, \omega)$, we obtain

$$
\sum_{k \in \mathbb{Z}^{d}} \int_{\phi\left(Q_{N}, \omega\right)}\left(\nabla \psi\left(\frac{1}{N} \phi^{-1}(z, \omega)-k\right)\right)^{T}\left(\nabla \phi\left(\phi^{-1}(z, \omega), \omega\right)\right)^{-1} A_{\mathrm{per}}\left(\phi^{-1}(z, \omega)\right)\left(p+\nabla w_{p}^{N}(z, \omega)\right) \mathrm{d} z=0,
$$

that we recast, using the definition $(6.19)$ of $w_{0, p}^{N}$, as

$$
\forall N \in \mathbb{N}^{\star}, \quad \sum_{k \in \mathbb{Z}^{d}} I_{k}^{N}(\omega)=0 \quad \text { a.s. }
$$

where

$I_{k}^{N}(\omega)=\int_{\frac{1}{N} \phi\left(Q_{N}, \omega\right)}\left(\nabla \psi\left(\frac{1}{N} \phi^{-1}(N z, \omega)-k\right)\right)^{T}\left(\nabla \phi\left(\phi^{-1}(N z, \omega), \omega\right)\right)^{-1} A_{\operatorname{per}}\left(\phi^{-1}(N z, \omega)\right)\left(p+\nabla w_{0, p}^{N}(z, \omega)\right) \mathrm{d} z$.

We claim that

$$
\forall k \in \mathbb{Z}^{d}, \quad \lim _{N \rightarrow \infty} I_{k}^{N}(\omega)=I_{k}^{\infty}(\omega) \quad \text { a.s. }
$$

where

$$
I_{k}^{\infty}(\omega):=\int_{\alpha Q}\left(\nabla \psi\left(\alpha^{-1} z-k\right)\right)^{T} \beta A^{\star}\left(p+\nabla w_{0, p}^{\infty}(z, \omega)\right)(\operatorname{det} \alpha)^{-1} \mathrm{~d} z,
$$

where the constant matrices $\alpha$ and $\beta$ are defined by (6.15) and (6.17).

Assume momentarily that (6.33) indeed holds. Then, as the sum in (6.32) has a finite number of terms, independently of $N$, we can pass to the limit $N \rightarrow \infty$ and obtain that

$$
\sum_{k \in \mathbb{Z}^{d}} I_{k}^{\infty}(\omega)=0 \quad \text { a.s. }
$$

which also reads

$$
\sum_{k \in \mathbb{Z}^{d}} \int_{\alpha Q}\left(\nabla \psi\left(\alpha^{-1} z-k\right)\right)^{T} \beta A^{\star}\left(p+\nabla w_{0, p}^{\infty}(z, \omega)\right)(\operatorname{det} \alpha)^{-1} \mathrm{~d} z=0 .
$$

Using the $\alpha Q$-periodicity of the function $w_{0, p}^{\infty}$ (shown in the above Step 2), we deduce that

$$
\int_{\mathbb{R}^{d}}(\nabla \psi(z))^{T} \beta A^{\star}\left(p+\nabla w_{0, p}^{\infty}(\alpha z, \omega)\right) \mathrm{d} z=0
$$

for all test functions $\psi \in \mathcal{D}\left(\mathbb{R}^{d}\right)$. We indeed have shown that

$$
-\operatorname{div}\left[\beta A^{\star} \nabla w_{0, p}^{\infty}(\alpha \cdot \omega)\right]=0 \text { in } \mathcal{D}^{\prime}\left(\mathbb{R}^{d}\right) .
$$

To conclude this Step, we are hence left with showing (6.33). Formally, this comes from the strong $L^{1}\left(\mathbb{R}^{d}\right)$ convergence of the indicator function $\mathbf{1}_{\frac{1}{N} \phi\left(Q_{N}, \omega\right)}$ towards $\mathbf{1}_{\alpha Q}$ and from the div-curl lemma. We indeed observe that the integrand in $I_{k}^{N}(\omega)$ is the product of $\left(\nabla \phi\left(\phi^{-1}(N z, \omega), \omega\right)\right)^{-T} \nabla \psi\left(\frac{1}{N} \phi^{-1}(N z, \omega)-k\right)$ with $A_{\text {per }}\left(\phi^{-1}(N z, \omega)\right)\left(p+\nabla w_{0, p}^{N}(z, \omega)\right)$. We will show in the sequel that the first factor is curl-free, whereas the second factor is divergence free. Using the div-curl lemma, this product converges (at least in the sense of distributions) towards the product of the weak limits of the two factors, which can be identified. One difficulty to make this argument rigorous is to find a fixed domain (independent of $N$ ) on which to apply the div-curl lemma. For that purpose, Lemma 6.2 is useful. 
We now proceed in details. Let $\eta>0$, and let $\mathcal{O}_{\eta} \subset \widetilde{\mathcal{O}}_{\eta}$ be two deterministic open sets such that $\overline{\widetilde{\mathcal{O}}_{\eta}}$ is proper subset of $\alpha Q, \overline{\mathcal{O}_{\eta}}$ is a proper subset of $\widetilde{\mathcal{O}}_{\eta}$, and

$$
\left|\alpha Q \backslash \widetilde{\mathcal{O}}_{\eta}\right| \leq \eta, \quad\left|\widetilde{\mathcal{O}}_{\eta} \backslash \mathcal{O}_{\eta}\right| \leq \eta .
$$

We then decompose $I_{k}^{N}(\omega)$ and $I_{k}^{\infty}(\omega)$ as follows: using (6.12) and (6.13), we write

$$
I_{k}^{N}(\omega)=I_{k, \eta}^{N}(\omega)+\mathcal{R}_{k, \eta}^{N}(\omega), \quad I_{k}^{\infty}(\omega)=I_{k, \eta}^{\infty}(\omega)+\mathcal{R}_{k, \eta}^{\infty}(\omega),
$$

with

$$
\begin{aligned}
I_{k, \eta}^{N}(\omega) & =\int_{\mathcal{O}_{\eta}}\left(\nabla \psi\left(\frac{1}{N} \phi^{-1}(N z, \omega)-k\right)\right)^{T}\left(\nabla \phi\left(\phi^{-1}(N z, \omega), \omega\right)\right)^{-1} A_{\text {per }}\left(\phi^{-1}(N z, \omega)\right)\left(p+\nabla w_{0, p}^{N}(z, \omega)\right) \mathrm{d} z, \\
I_{k, \eta}^{\infty}(\omega) & =\int_{\mathcal{O}_{\eta}}\left(\nabla \psi\left(\alpha^{-1} z-k\right)\right)^{T} \beta A^{\star}\left(p+\nabla w_{0, p}^{\infty}(z, \omega)\right)(\operatorname{det} \alpha)^{-1} \mathrm{~d} z \\
\mathcal{R}_{k, \eta}^{N}(\omega)= & \int_{\widetilde{Q}(\omega)}\left(\mathbf{1}_{\frac{1}{N} \phi\left(Q_{N}, \omega\right)}(z)-\mathbf{1}_{\mathcal{O}_{\eta}}(z)\right)\left(\nabla \psi\left(\frac{1}{N} \phi^{-1}(N z, \omega)-k\right)\right)^{T} \\
& \times\left(\nabla \phi\left(\phi^{-1}(N z, \omega), \omega\right)\right)^{-1} A_{\text {per }}\left(\phi^{-1}(N z, \omega)\right)\left(p+\nabla w_{0, p}^{N}(z, \omega)\right) \mathrm{d} z, \\
\mathcal{R}_{k, \eta}^{\infty}(\omega)= & \int_{\alpha Q \backslash \mathcal{O}_{\eta}}\left(\nabla \psi\left(\alpha^{-1} z-k\right)\right)^{T} \beta A^{\star}\left(p+\nabla w_{0, p}^{\infty}(z, \omega)\right)(\operatorname{det} \alpha)^{-1} \mathrm{~d} z .
\end{aligned}
$$

To use the div-curl lemma, we need to further decompose $I_{k, \eta}^{N}(\omega)$ and $I_{k, \eta}^{\infty}(\omega)$. Introducing a smooth truncation function $\xi \in \mathcal{D}\left(\widetilde{\mathcal{O}}_{\eta}\right)$ such that $0 \leq \xi(x) \leq 1$ a.e. and $\xi \equiv 1$ on $\mathcal{O}_{\eta}$, we write that

$$
I_{k, \eta}^{N}(\omega)=\widetilde{I}_{k, \eta}^{N}(\omega)-C_{\eta}^{N}(\omega), \quad I_{k, \eta}^{\infty}(\omega)=\widetilde{I}_{k, \eta}^{\infty}(\omega)-C_{\eta}^{\infty}(\omega)
$$

where

$$
\begin{aligned}
C_{\eta}^{\infty}(\omega)= & \int_{\widetilde{\mathcal{O}}_{\eta} \backslash \mathcal{O}_{\eta}} \xi(z)\left(\nabla \psi\left(\alpha^{-1} z-k\right)\right)^{T} \beta A^{\star}\left(p+\nabla w_{0, p}^{\infty}(z, \omega)\right)(\operatorname{det} \alpha)^{-1} \mathrm{~d} z \\
\widetilde{I}_{k, \eta}^{\infty}(\omega)= & \int_{\widetilde{\mathcal{O}}_{\eta}} \xi(z)\left(\nabla \psi\left(\alpha^{-1} z-k\right)\right)^{T} \beta A^{\star}\left(p+\nabla w_{0, p}^{\infty}(z, \omega)\right)(\operatorname{det} \alpha)^{-1} \mathrm{~d} z \\
\widetilde{I}_{k, \eta}^{N}(\omega)= & \int_{\widetilde{\mathcal{O}}_{\eta}} \xi(z)\left(\nabla \psi\left(\frac{1}{N} \phi^{-1}(N z, \omega)-k\right)\right)^{T} \\
& \times\left(\nabla \phi\left(\phi^{-1}(N z, \omega), \omega\right)\right)^{-1} A_{\text {per }}\left(\phi^{-1}(N z, \omega)\right)\left(p+\nabla w_{0, p}^{N}(z, \omega)\right) \mathrm{d} z
\end{aligned}
$$

and

$$
\begin{aligned}
C_{\eta}^{N}(\omega)= & \int_{\widetilde{\mathcal{O}}_{\eta} \backslash \mathcal{O}_{\eta}} \xi(z)\left(\nabla \psi\left(\frac{1}{N} \phi^{-1}(N z, \omega)-k\right)\right)^{T}\left(\nabla \phi\left(\phi^{-1}(N z, \omega), \omega\right)\right)^{-1} \\
& \times A_{\text {per }}\left(\phi^{-1}(N z, \omega)\right)\left(p+\nabla w_{0, p}^{N}(z, \omega)\right) \mathrm{d} z .
\end{aligned}
$$

We first bound from above $C_{\eta}^{N}(\omega), C_{\eta}^{\infty}(\omega), \mathcal{R}_{k, \eta}^{N}(\omega)$ and $\mathcal{R}_{k, \eta}^{\infty}(\omega)$. As $|\xi| \leq 1$, we see that

$$
\begin{aligned}
\left|C_{\eta}^{N}(\omega)\right| & \leq\|\nabla \psi\|_{L^{\infty}}\left\|(\nabla \phi)^{-1}(\cdot, \omega)\right\|_{L^{\infty}}\left\|A_{\mathrm{per}}\right\|_{L^{\infty}}\left|\widetilde{\mathcal{O}}_{\eta} \backslash \mathcal{O}_{\eta}\right|^{1 / 2}\left\|p+\nabla w_{0, p}^{N}(\cdot, \omega)\right\|_{L^{2}\left(\widetilde{\mathcal{O}}_{\eta} \backslash \mathcal{O}_{\eta}\right)} \\
& \leq C \sqrt{\eta}\left\|p+\nabla w_{0, p}^{N}(\cdot, \omega)\right\|_{L^{2}(\widetilde{Q}(\omega))}
\end{aligned}
$$


where, in the second line, we have used (6.8), (6.35) and the fact that $\widetilde{\mathcal{O}}_{\eta} \backslash \mathcal{O}_{\eta} \subset \widetilde{\mathcal{O}}_{\eta} \subset \alpha Q \subset \widetilde{Q}(\omega)$ (see (6.13)). Now using (6.20), we deduce that there exists $C(\omega)$, independent of $\eta$ and $N$, such that

$$
\forall N \in \mathbb{N}^{\star}, \quad\left|C_{\eta}^{N}(\omega)\right| \leq C(\omega) \sqrt{\eta} .
$$

We likewise obtain that

$$
\left|C_{\eta}^{\infty}(\omega)\right| \leq C(\omega) \sqrt{\eta} \quad \text { and } \quad\left|\mathcal{R}_{k, \eta}^{\infty}(\omega)\right| \leq C(\omega) \sqrt{\eta} .
$$

Now turning to $\mathcal{R}_{k, \eta}^{N}(\omega)$, we obtain, using similar arguments, that

$$
\left|\mathcal{R}_{k, \eta}^{N}(\omega)\right| \leq C\left\|\mathbf{1}_{\frac{1}{N} \phi\left(Q_{N}, \omega\right)}-\mathbf{1}_{\mathcal{O}_{\eta}}\right\|_{L^{2}(\widetilde{Q}(\omega))}\left\|p+\nabla w_{0, p}^{N}(\cdot, \omega)\right\|_{L^{2}(\widetilde{Q}(\omega))} .
$$

Using (6.20), a triangle inequality and (6.35), we deduce that

$$
\left|\mathcal{R}_{k, \eta}^{N}(\omega)\right| \leq C(\omega)\left(\left\|\mathbf{1}_{\frac{1}{N} \phi\left(Q_{N}, \omega\right)}-\mathbf{1}_{\alpha Q}\right\|_{L^{2}(\widetilde{Q}(\omega))}+\sqrt{\eta}\right) .
$$

Recall now that, in view of (6.7), we have

$$
\lim _{N \rightarrow \infty}\left\|\mathbf{1}_{\frac{1}{N} \phi\left(Q_{N}, \omega\right)}-\mathbf{1}_{\alpha Q}\right\|_{L^{2}(\widetilde{Q}(\omega))}=0 \text { a.s. }
$$

We eventually estimate $\widetilde{I}_{k, \eta}^{N}(\omega)-\widetilde{I}_{k, \eta}^{\infty}(\omega)$ using the div-curl lemma. The compact $\widetilde{\widetilde{\mathcal{O}}_{\eta}}$ being a proper subset of $\alpha Q$, we infer from Lemma 6.2 that there exists $N_{0}(\omega)$ such that for all $N \geq N_{0}(\omega), \widetilde{\mathcal{O}}_{\eta} \subset \frac{1}{N} \phi\left(Q_{N}, \omega\right)$. We then deduce from (3.9) that, for any $N \geq N_{0}(\omega)$,

$$
-\operatorname{div}\left[A_{\text {per }}\left(\phi^{-1}(N z, \omega)\right)\left(p+\nabla w_{0, p}^{N}(z, \omega)\right)\right]=0 \quad \text { in } \mathcal{D}^{\prime}\left(\widetilde{\mathcal{O}}_{\eta}\right)
$$

Using (6.21), we can thus apply Theorem 6.1 on the domain $\widetilde{\mathcal{O}}_{\eta}$, and obtain that

$$
A_{\text {per }}\left(\phi^{-1}(N z, \omega)\right)\left(p+\nabla w_{0, p}^{N}(z, \omega)\right) \rightarrow A^{\star}\left(p+\nabla w_{0, p}^{\infty}(z, \omega)\right) \quad \text { weakly in } L^{2}\left(\widetilde{\mathcal{O}}_{\eta}\right) .
$$

From the proof of Lemme 2.2 of [7] we know that $\frac{1}{N} \phi^{-1}(N z, \omega)$ strongly converges in $L_{\text {loc }}^{\infty}\left(\mathbb{R}^{d}\right)$ toward $\alpha^{-1} z$. As, by definition, $\nabla \psi \in \mathcal{C}^{\infty}\left(\mathbb{R}^{d}\right)$, we obtain that

$$
\nabla \psi\left(\frac{1}{N} \phi^{-1}(N z, \omega)-k\right) \rightarrow \nabla \psi\left(\alpha^{-1} z-k\right) \quad \text { strongly in } L_{\mathrm{loc}}^{\infty}\left(\mathbb{R}^{d}\right) .
$$

Since $(\nabla \phi)^{-1}$ is stationary, we infer from Lemme 2.2 of [7] that

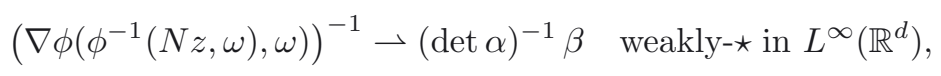

where the matrix $\beta$ is defined by (6.17). As $\widetilde{\mathcal{O}}_{\eta}$ is a bounded open set of $\mathbb{R}^{d}$, we deduce from (6.44) and (6.45) that

$$
\left(\nabla \psi\left(\frac{1}{N} \phi^{-1}(N z, \omega)-k\right)\right)^{T}\left(\nabla \phi\left(\phi^{-1}(N z, \omega), \omega\right)\right)^{-1} \rightarrow(\operatorname{det} \alpha)^{-1}\left(\nabla \psi\left(\alpha^{-1} z-k\right)\right)^{T} \beta \quad \text { weakly in } L^{2}\left(\widetilde{\mathcal{O}}_{\eta}\right) .
$$


We eventually note that

$$
\left(\nabla \phi\left(\phi^{-1}(N z, \omega), \omega\right)\right)^{-T} \nabla \psi\left(\frac{1}{N} \phi^{-1}(N z, \omega)-k\right) \text { is curl-free, }
$$

as this vector is the gradient of $\psi\left(\frac{1}{N} \phi^{-1}(N z, \omega)-k\right)$. Collecting $(6.42),(6.43),(6.46)$ and $(6.47)$, we are in position to apply the div-curl lemma, see for instance Lemme 1.1 page 4 of [15]. We thus obtain that

$$
\forall \eta, \quad \lim _{N \rightarrow \infty} \widetilde{I}_{k, \eta}^{N}(\omega)=\widetilde{I}_{k, \eta}^{\infty}(\omega) \quad \text { a.s. }
$$

Collecting (6.36), (6.37), (6.38), (6.39), (6.40), (6.41) and (6.48), we deduce the claim (6.33). This concludes this Step.

Step 4. Conclusion

Collecting the conclusion of Step 2 and $(6.34)$, we have shown that the function $w_{0, p}^{\infty}(\cdot, \omega)$ solves the problem

$$
-\operatorname{div}\left[\beta A^{\star} \nabla w_{0, p}^{\infty}(\alpha \cdot, \omega)\right]=0 \text { in } \mathcal{D}^{\prime}\left(\mathbb{R}^{d}\right), \quad w_{0, p}^{\infty}(\alpha \cdot, \omega) \text { is } Q \text {-periodic. }
$$

The function $g(x, \omega)=w_{0, p}^{\infty}(\alpha x, \omega)$ is thus $Q$-periodic and satisfies

$$
-\operatorname{div}\left[\beta A^{\star} \alpha^{-T} \nabla g(\cdot, \omega)\right]=0 \text { in } \mathcal{D}^{\prime}\left(\mathbb{R}^{d}\right) .
$$

We know from Lemma 6.5 that the matrix $\beta A^{\star} \alpha^{-T}$ is coercive. The above equation has thus a unique solution (up to the addition of a random constant), hence $\nabla g \equiv 0$, which implies that $\nabla w_{0, p}^{\infty} \equiv 0$. We thus deduce from (6.43) that

$$
A_{\text {per }}\left(\phi^{-1}(N \cdot, \omega)\right)\left(p+\nabla w_{0, p}^{N}(\cdot, \omega)\right) \rightarrow A^{\star} p \quad \text { as } N \rightarrow \infty \text {, weakly in } L^{2}\left(\mathcal{O}_{\eta}\right) .
$$

We are now in position to prove the convergence of the approximation described in Section 3.2. We infer from (3.11) that

with

$$
\left[B_{N}^{\star}(\omega)\right]_{i j}=R_{1, \eta}^{N}(\omega)+R_{2, \eta}^{N}(\omega)
$$

$$
\begin{aligned}
& R_{1, \eta}^{N}(\omega)=\int_{\widetilde{Q}(\omega)}\left(\mathbf{1}_{\frac{1}{N} \phi\left(Q_{N}, \omega\right)}(x)-\mathbf{1}_{\mathcal{O}_{\eta}}(x)\right) e_{i}^{T} A_{\text {per }}\left(\phi^{-1}(N x, \omega)\right)\left(e_{j}+\nabla w_{0, e_{j}}^{N}(x, \omega)\right) \mathrm{d} x, \\
& R_{2, \eta}^{N}(\omega)=\int_{\mathcal{O}_{\eta}} e_{i}^{T} A_{\text {per }}\left(\phi^{-1}(N x, \omega)\right)\left(e_{j}+\nabla w_{0, e_{j}}^{N}(x, \omega)\right) \mathrm{d} x,
\end{aligned}
$$

where we have used that $\mathcal{O}_{\eta} \subset \alpha Q,(6.12)$ and (6.13). We deduce from (6.49) that

$$
\forall \eta, \quad \lim _{N \rightarrow \infty} R_{2, \eta}^{N}(\omega)=\left|\mathcal{O}_{\eta}\right|\left[A^{\star}\right]_{i j} \quad \text { a.s. },
$$

hence, in view of (6.35),

$$
\lim _{\eta \rightarrow 0} \lim _{N \rightarrow \infty} R_{2, \eta}^{N}(\omega)=|\alpha Q|\left[A^{\star}\right]_{i j} \quad \text { a.s. }
$$

Turning to $R_{1, \eta}^{N}(\omega)$, we deduce from (6.20) and (6.35) that

$$
\left|R_{1, \eta}^{N}(\omega)\right| \leq C(\omega)\left\|\mathbf{1}_{\frac{1}{N} \phi\left(Q_{N}, \omega\right)}-\mathbf{1}_{\mathcal{O}_{\eta}}\right\|_{L^{2}(\widetilde{Q}(\omega))} \leq C(\omega)\left(\left\|\mathbf{1}_{\frac{1}{N} \phi\left(Q_{N}, \omega\right)}-\mathbf{1}_{\alpha Q}\right\|_{L^{2}(\widetilde{Q}(\omega))}+\sqrt{\eta}\right),
$$

hence, in view of (6.41),

$$
\lim _{\eta \rightarrow 0} \lim _{N \rightarrow \infty} R_{1, \eta}^{N}(\omega)=0 \quad \text { a.s. }
$$

Collecting (6.50), (6.51) and (6.52), we obtain

$$
\lim _{N \rightarrow \infty}\left[B_{N}^{\star}(\omega)\right]_{i j}=|\alpha Q| A_{i j}^{\star} \quad \text { a.s. }
$$

We then deduce from (3.10) the claimed convergence. This concludes the proof of Theorem 3.4. 


\subsection{Proof of Lemma 6.5}

We first show that

the homogenized matrix $A^{\star}$ defined by $(2.8)$ is coercive.

For any $p \in \mathbb{R}^{d}$, we indeed have

$$
\begin{aligned}
p^{T} A^{\star} p & =(\operatorname{det} \alpha)^{-1} \mathbb{E}\left(\int_{\phi(Q, \cdot)} p^{T} A_{\text {per }}\left(\phi^{-1}(y, \cdot)\right)\left(p+\nabla w_{p}(y, \cdot)\right) \mathrm{d} y\right) \\
& =(\operatorname{det} \alpha)^{-1} \mathbb{E}\left(\int_{\phi(Q, \cdot)}\left(p+\nabla w_{p}(y, \cdot)\right)^{T} A_{\operatorname{per}}\left(\phi^{-1}(y, \cdot)\right)\left(p+\nabla w_{p}(y, \cdot)\right) \mathrm{d} y\right)
\end{aligned}
$$

where the last line is obtained using (3.8) and the arguments presented in the existence, proof of Théorème 1.2 of [7]. The matrix $A_{\text {per }}$ being coercive (see (2.3)), we deduce that there exists $C>0$ such that, for any $p \in \mathbb{R}^{d}$,

$$
\begin{aligned}
p^{T} A^{\star} p & \geq a_{-}(\operatorname{det} \alpha)^{-1} \mathbb{E}\left(\int_{\phi(Q, \cdot)}\left(p+\nabla w_{p}(y, \cdot)\right)^{T}\left(p+\nabla w_{p}(y, \cdot)\right) \mathrm{d} y\right) \\
& \geq C\left[\mathbb{E}\left(\int_{\phi(Q, \cdot)} p+\nabla w_{p}(y, \cdot)\right)\right]^{2} \quad \text { (Cauchy-Schwarz inequality) } \\
& \geq C p^{T} p \quad(\text { using third line of }(2.9)) .
\end{aligned}
$$

This proves (6.53).

We now claim that the matrix $\beta$ defined by (6.17) satisfies

$$
\beta=\operatorname{det} \alpha \alpha^{-1} \text {. }
$$

This is obvious in dimension $d=1$, and also in dimension $d=2$, using the explicit formula of the inverse of a $2 \times 2$ matrix. In dimension $d \geq 3$, we observe that $\beta=\mathbb{E}\left[\int_{Q} \operatorname{adj} \nabla \phi\right]$, where $\operatorname{adj} \nabla \phi$ is the adjugate matrix (i.e. the transpose of the matrix of cofactors) of $\nabla \phi$. Introduce $\theta_{\varepsilon}(x, \omega)=\varepsilon \phi\left(\frac{x}{\varepsilon}, \omega\right)-\varepsilon \phi(0, \omega)$. We see from Lemme 2.1 of [7] that the function $\theta_{\varepsilon}(x, \omega)$ weakly converges to $\mathbb{E}\left[\int_{Q} \nabla \phi\right] x$ in $L_{\text {loc }}^{\infty}\left(\mathbb{R}^{d}\right)$ a.s. We then deduce from Corollary 1 of [14] and (6.16) (see also Corollary 6.2.2 of [4] for the specific case $d=3$ ) that adj $\nabla \theta_{\varepsilon}$ weakly converges to adj $\mathbb{E}\left[\int_{Q} \nabla \phi\right]$, that is

$$
\operatorname{adj} \nabla \phi\left(\frac{x}{\varepsilon}, \omega\right) \text { weakly converges to adj } \mathbb{E}\left[\int_{Q} \nabla \phi\right] .
$$

In addition, the matrix $\nabla \phi$ being stationary, the ergodic theorem implies that

$$
\operatorname{adj} \nabla \phi\left(\frac{x}{\varepsilon}, \omega\right) \text { weakly converges to } \mathbb{E}\left[\int_{Q} \operatorname{adj} \nabla \phi\right] .
$$

Collecting (6.55) and (6.56), we deduce that $\mathbb{E}\left[\int_{Q} \operatorname{adj} \nabla \phi\right]=\operatorname{adj} \mathbb{E}\left[\int_{Q} \nabla \phi\right]$, from which we readily infer (6.54).

We are now in position to prove Lemma 6.5. Using (6.54) and (6.53), we indeed see that there exists $C>0$ such that, for any $p \in \mathbb{R}^{d}$, we have

$$
p^{T} \beta A^{\star} \alpha^{-T} p=\operatorname{det} \alpha p^{T} \alpha^{-1} A^{\star} \alpha^{-T} p \geq C p^{T} \alpha^{-1} \alpha^{-T} p .
$$


Since $\operatorname{det} \alpha>0$, we see that the matrix $\alpha^{-1} \alpha^{-T}$ is symmetrix positive definite, which concludes the proof of Lemma 6.5.

Acknowledgements. We thank Claude Le Bris and Xavier Blanc for stimulating discussions and useful comments on a preliminary version of this article. We are thankful to Xavier Blanc for pointing out the reference [14], and to the anonymous referee for his/her comments. This work is partially supported by ONR under Grant N00014-12-1-0383 and by EOARD under Grant FA8655-13-1-3061.

\section{REFERENCES}

[1] A. Anantharaman, R. Costaouec, C. Le Bris, F. Legoll and F. Thomines, Introduction to numerical stochastic homogenization and the related computational challenges: some recent developments. In vol. 22 of Lect. Not. Ser., edited by W. Bao and Q. Du. Institute for Mathematical Sciences, National University of Singapore, (2011) 197-272.

[2] G. Bal, J. Garnier, Y. Gu and W. Jing, Corrector theory for elliptic equations with long-range correlated random potential. Asymptot. Anal. 77 (2012) 123-145.

[3] G. Bal, J. Garnier, S. Motsch and V. Perrier, Random integrals and correctors in homogenization. Asymptot. Anal. 59 (2008) $1-26$.

[4] J.M. Ball, Convexity conditions and existence theorems in nonlinear elasticity. Arch. Ration. Mech. Anal. 63 (1977) $337-403$.

[5] A. Bensoussan, J.-L. Lions and G. Papanicolaou, Asymptotic analysis for periodic structures, vol. 5 of Studi. Math. Appl. North-Holland Publishing Co., Amsterdam-New York (1978).

[6] P. Billingsley, Convergence of Probability Measures. John Wiley \& Sons Inc (1968).

[7] X. Blanc, C. Le Bris and P.-L. Lions, Une variante de la théorie de l'homogénéisation stochastique des opérateurs elliptiques [A variant of stochastic homogenization theory for elliptic operators]. C. R. Acad. Sci. Série I 343 (2006) $717-724$.

[8] X. Blanc, C. Le Bris and P.-L. Lions, Stochastic homogenization and random lattices. J. Math. Pures Appl. 88 (2007) $34-63$.

[9] A. Bourgeat and A. Piatnitski, Estimates in probability of the residual between the random and the homogenized solutions of one-dimensional second-order operator. Asymptot. Anal. 21 (1999) 303-315.

[10] A. Bourgeat and A. Piatnitski, Approximation of effective coefficients in stochastic homogenization. Ann Inst. Henri PoincaréPR 40 (2004) 153-165.

[11] D. Cioranescu and P. Donato, An introduction to homogenization, vol. 17 of Oxford Lect. Ser. Math. Appl. Oxford University Press, New York (1999).

[12] R. Costaouec, C. Le Bris and F. Legoll, Approximation numérique d'une classe de problèmes en homogénéisation stochastique [Numerical approximation of a class of problems in stochastic homogenization]. C. R. Acad. Sci. Série I 348 (2010) $99-103$.

[13] B. Engquist and P.E. Souganidis, Asymptotic and numerical homogenization. Acta Numerica 17 (2008) $147-190$.

[14] D. Henao and C. Mora-Corral, Invertibility and weak continuity of the determinant for the modelling of cavitation and fracture in nonlinear elasticity. Arch. Ration. Mech. Anal. 197 (2010) 619-655.

[15] V.V. Jikov, S.M. Kozlov and O.A. Oleinik, Homogenization of differential operators and integral functionals. Springer-Verlag (1994).

[16] U. Krengel, Ergodic theorems, vol. 6 of De Gruyter Studies in Mathematics. De Gruyter (1985).

[17] G.C. Papanicolaou and S.R.S. Varadhan, Boundary value problems with rapidly oscillating random coefficients, in Proc. Colloq. on Random Fields: Rigorous Results in Statistical Mechanics and Quantum Field Theory (1979). In vol. 10 of Colloquia Mathematica Societatis Janos Bolyai, edited by J. Fritz, J.L. Lebaritz and D. Szasz. North-Holland (1981) 835-873.

[18] A.N. Shiryaev, Probability, vol. 95 of Graduate Texts in Mathematics. Springer (1984).

[19] A.A. Tempel'man, Ergodic theorems for general dynamical systems. Trudy Moskov. Mat. Obsc. 26 (1972) $94-132$.

[20] V.V. Yurinskii, Averaging of symmetric diffusion in random medium. Sibirskii Mat. Zh. 27 (1986) 167-180. 